\title{
Technical Justification for a Request to Reclassify the Former CCC/USDA Facility at Canada, Kansas
}

\section{Environmental Science Division}




\begin{abstract}
About Argonne National Laboratory
Argonne is a U.S. Department of Energy laboratory managed by UChicago Argonne, LLC under contract DE-AC02-06CH11357. The Laboratory's main facility is outside Chicago, at 9700 South Cass Avenue, Argonne, Illinois 60439. For information about Argonne, see www.anl.gov.
\end{abstract}

\title{
Availability of This Report
}

This report is available, at no cost, at http://www.osti.gov/bridge. It is also available on paper to the U.S. Department of Energy and its contractors, for a processing fee, from:

U.S. Department of Energy

Office of Scientific and Technical Information

P.O. Box 62

Oak Ridge, TN 37831-0062

phone (865) 576-8401

fax (865) 576-5728

reports@adonis.osti.gov

\section{Disclaimer}

This report was prepared as an account of work sponsored by an agency of the United States Government. Reference herein to any specific commercial product, process, or service by trade name, trademark, manufacturer, or otherwise, does not necessarily constitute or imply its endorsement, recommendation, or favoring by the United States Government or any agency thereof. The views and opinions of document authors expressed herein do not necessarily state or reflect those of the United States Government or any agency thereof, Argonne National Laboratory, or UChicago Argonne, LLC. 


\section{Technical Justification for a Request to Reclassify the Former CCC/USDA Facility at Canada, Kansas}

by

Applied Geosciences and Environmental Management Section

Environmental Science Division, Argonne National Laboratory

August 2007

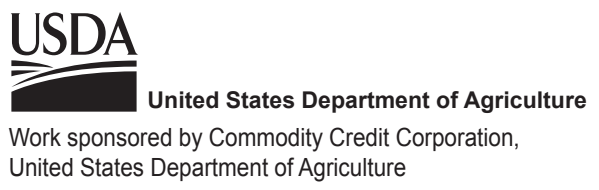




\section{Contents}

Notation.

Executive Summary ES-1

1 Introduction

2 Background

2.1 Site Location and Description

$2-1$

2.2 Grain Storage Operations and Property History

3 Carbon Tetrachloride Contamination at Canada

3.1 Identification of Contamination and Removal Action

3.2 Contaminant History and Previous Investigations

4 Conclusions

5 References...

Apendix A: Well Registration Forms and Drilling Records for Canada Area Wells

Appendix B: Geology of the Canada Area, Marion County.

B-1

Appendix C: Property Records Related to the Former CCC/USDA Facility at Canada

C-1

\section{Figures}

2.1 Location of Canada, Kansas.

2.2 Plat of Canada, Kansas.

2.3 Grain storage structures at Canada in 1956, with property boundaries

2.4 Grain storage structures at Canada in 1991, with locations of 1956 structures shown in Figure 2.3 and property boundaries

2.5 Current configuration of the Canada site 
3.1 Analytical results for carbon tetrachloride in groundwater samples collected in 2007.

3.2 Analytical results for soil samples collected in November 1999

3.3 Structures at the Canada site, 2007 groundwater elevations with interpreted flow direction, and approximate locations of KDHE monitoring wells and private wells with 2007 monitoring results.

3.4 Structures at the Canada site, 2005 groundwater elevations with interpreted flow direction, and approximate locations of KDHE monitoring wells and private wells with 2005 monitoring results

3.5 Structures at the Canada site, 2003 groundwater elevations with interpreted flow direction, and approximate locations of KDHE monitoring wells and private wells with 2003 monitoring results.

3.6 Structures at the Canada site, 2001 groundwater elevations with interpreted flow direction, and approximate locations of KDHE monitoring wells and private wells with 2001 monitoring results.

A.1 Locations of registered wells in the Canada area

B.1 Stratigraphic units that crop out in Marion County, Kansas, and the construction materials of each.

B.2 Generalized surface geologic map of Marion County, showing the location of the town of Canada.

B.3 Location of geologic cross section A-A' in central Marion County.....

B.4 Interpretive west-to-east geologic cross section A-A', Marion County, Kansas

C.1 CCC/USDA lease, 1954 to 1964

C.2 CCC/USDA lease, 1964 to 1974

C.3 Sale of property by Burkholder to Canada Grain Co., 1965

C-6

C.4 Foreclosure on Canada Grain Co., 1976

C-8

C.5 Warranty deed, D\&R Grain to Countryside Grain, 1978

C-10

C.6 Warranty deed, Janzen Farm Service to Cooperative Grain and Supply, 1991 C-11 
Canada, Kansas, Technical Justification for Request to Reclassify

Version 01, 08/17/07

\section{Tables}

3.1 Chronological history of groundwater sampling and analysis at Canada ....................... 3-5

3.2 Water Level data for the Canada site ………….................................................. 3-8

3.3 Survey data for the Canada site............................................................................ $3-9$

3.4 Historical results of groundwater sampling and analysis for individual wells

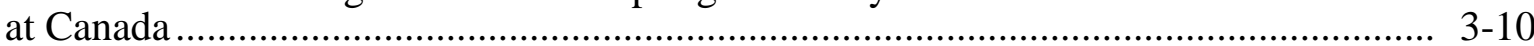




\section{Notation}

AMSL above mean sea level

BER Bureau of Environmental Remediation (KDHE)

BGL below ground level

CCC Commodity Credit Corporation

CERCLIS Comprehensive Environmental Response, Compensation, and Liability Information System

CGS Cooperative Grain and Supply

DCA 1,1-dichloroethane

EDB ethylene dibromide

$\mathrm{ft} \quad$ foot (feet)

FSA Farm Service Agency

gpm gallon(s) per minute

IGA Intergovernmental Agreement

ISL Identified Sites List (KDHE)

KDHE Kansas Department of Health and Environment

KGS Kansas Geological Survey

$\mu \mathrm{g} / \mathrm{kg} \quad$ microgram(s) per kilogram

$\mu \mathrm{g} / \mathrm{L} \quad$ microgram(s) per liter

MCL maximum contaminant level

$\mathrm{mg} / \mathrm{L} \quad$ milligram(s) per liter

mi mile(s)

MTBE methyl tert-butyl ether

RWD Rural Water District

USACE U.S. Army Corps of Engineers

USDA U.S. Department of Agriculture

USGS U.S. Geological Survey

WWC-5 water well record form 


\section{Technical Justification for a Request to Reclassify the Former CCC/USDA Facility at Canada, Kansas}

\section{Executive Summary}

Contamination in groundwater at Canada, Kansas, was discovered in 1997, during limited private well sampling near former grain storage facilities of the Commodity Credit Corporation, U.S. Department of Agriculture (CCC/USDA). Subsequent investigations by the Kansas Department of Health and Environment (KDHE) confirmed carbon tetrachloride and nitrate concentrations in groundwater above the respective maximum contaminant levels (MCLs) of $5.0 \mu \mathrm{g} / \mathrm{L}$ and $10.0 \mathrm{mg} / \mathrm{L}$. The KDHE investigations identified both the former CCC/USDA grain storage facility and a private grain storage facility as likely sources for the carbon tetrachloride contamination. The CCC/USDA funded extension of a rural water district line to provide a permanent alternate water supply, and the KDHE has conducted long-term monitoring under the State Water Plan.

This document presents an analysis of the available information for the Canada site, acquired in previous investigations and the long-term KDHE monitoring. This analysis forms the technical justification for a request to reclassify the former CCC/USDA grain storage facility at Canada as a site requiring no further action under the Intergovernmental Agreement (IGA) between the KDHE and the USDA's Farm Service Agency.

The KDHE's long-term water level monitoring results indicate a consistent groundwater flow direction to the east-southeast. Consequently, the wells with the highest overall concentrations of carbon tetrachloride are downgradient from the private grain storage facility but not downgradient from the former CCC/USDA facility.

The KDHE criterion for reclassification of a site is that contamination there should not pose an unacceptable risk, on the basis of analytical results for four consecutive, equally timed, sequenced sampling episodes over a period of no less than two years. In seven KDHE sampling events over a period of six years (2001-2007), the concentrations of carbon tetrachloride in the monitoring well on the former CCC/USDA property at Canada have ranged from $0.63 \mu \mathrm{g} / \mathrm{L}$ to $2.9 \mu \mathrm{g} / \mathrm{L}$. These seven results are all below the MCL of $5.0 \mu \mathrm{g} / \mathrm{L}$, and they demonstrate that groundwater on the former CCC/USDA property has met the standard for carbon tetrachloride in drinking water since 2001 and does not pose a significant human health threat. Except for a twoyear interval between sampling events in December 2003 and December 2005, the monitoring 
occurred at approximately annual intervals. The results warrant a request for reclassification of the Canada site to Resolved status on the KDHE's Identified Sites List, under Section III (Other Considerations) of KDHE policy BER-RS-024, Rev. 2001. 


\section{Introduction}

Contamination at the grain storage facility formerly operated by the Commodity Credit Corporation of the U.S. Department of Agriculture (CCC/USDA) at Canada, Kansas, was discovered in October 1997, during limited private well sampling in conjunction with an inventory of such facilities. The Kansas Department of Health and Environment (KDHE) conducted investigations at Canada in 1998-2001 and confirmed carbon tetrachloride and nitrate concentrations in groundwater above the respective maximum contaminant levels (MCLs) of $5.0 \mu \mathrm{g} / \mathrm{L}$ and $10 \mathrm{mg} / \mathrm{L}$. The affected private lawn and garden wells are no longer used to supply drinking water, and carbon tetrachloride impacts above the MCL are localized.

The KDHE investigations identified both the former CCC/USDA grain bin location and a private grain storage facility as likely sources of the carbon tetrachloride contamination. After the identification of carbon tetrachloride and nitrate contamination at concentrations above the respective MCLs, the CCC/USDA funded extension of the Marion Rural Water District (RWD) \#4 line to provide a permanent alternate water supply to affected residents.

Because of the limited exposure risk, the KDHE assigned the Canada site to long-term monitoring status under the State Water Plan. The site is presently classified under the Intergovernmental Agreement (IGA) between the USDA's Farm Service Agency (FSA) and the KDHE as a site that the FSA considers to require no further action on its part, but for which the KDHE has not yet made a determination.

This report presents an analysis of long-term monitoring results for 2001-2007 (KDHE 2007a) and results of prior site investigations. The analysis indicates that the former CCC/USDA grain storage facility in the southern portion of town is not the most plausible source of the carbon tetrachloride contamination at levels above the MCL. Rather, the analytical data and the associated groundwater flow direction data indicate that the private grain storage locations in the northern portion of town are the more likely source of the downgradient carbon tetrachloride contamination. The information presented here forms the technical justification for a request to reclassify the former CCC/USDA grain storage facility at Canada as a site requiring no further action under the IGA. 


\section{Background}

\subsection{Site Location and Description}

Canada, Kansas, is a small, unincorporated rural community in Marion County, in central Kansas (Figure 2.1). Canada is located near U.S. Highway 56, approximately $5 \mathrm{mi}$ east of Hillsboro (population 2,704) and $5 \mathrm{mi}$ west of Marion (population 1,906). Wichita is about $40 \mathrm{mi}$ to the south. Canada lies within Section 33, Township 19 South, Range 3 East, at approximate latitude $38^{\circ} 21^{\prime} 16^{\prime \prime}$ North and longitude $97^{\circ} 06^{\prime} 41^{\prime \prime}$ West (USGS 1985).

Canada has a population of approximately 24. The Canada facility of Cooperative Grain and Supply (CGS), Hillsboro, Kansas, currently occupies the co-op property in the central portion of the community and provides seasonal grain storage for area farmers (Figure 2.2). A spur of the Atchison, Topeka, and Santa Fe Railroad (now removed) was formerly located north of the co-op. The CCC/USDA formerly operated a grain storage facility on what is now the southern portion of the co-op property. Ten homes are adjacent to the co-op, to the east and west. A gasoline station and bait shop are west of the co-op.

Canada lies within the Cottonwood River drainage basin (Figure 2.1), approximately $1 \mathrm{mi}$ west of the confluence of the North Cottonwood River (north of town) and the South Cottonwood River (south of town). An intermittent stream flows south-southeast through the town, discharging into the South Cottonwood River. No surface water targets were identified within the 15-mi downstream target distance limit (KDHE 1999a). Until the construction of Marion Lake, approximately 1 mi north of Canada, the town was within the 100-year floodplain of the river.

At Canada, screened intervals of domestic wells for which records are available (Appendix A) are in the range of 45-65 ft BGL (below ground level). One well (DW24 [Schroeder] Figure 2.2) is also screened in an upper zone at 25-35 ft BGL. Water is potentially produced from solution joints in the limestone units of the Permian Nolans Formation. The screened depths suggest that multiple water-bearing zones are present at Canada. A review of the geology of Marion County, Kansas, in the Canada area, is in Appendix B.

Evidence gathered from earlier interviews with local residents suggested a groundwater flow direction to the east-southeast (KDHE 1998). This flow direction is consistent with data 
collected during the KDHE's long-term monitoring (2001-2007), as discussed in detail in Section 3.2. Topographic features in the area also support this interpretation. For example, a local flow direction to the southeast is consistent with the erosion by the South Cottonwood River of Permian rocks $1.25 \mathrm{mi}$ southeast of Canada. Downcutting by the river extends to about $40-50 \mathrm{ft}$ below the typical elevation of the ground surface at Canada. Another factor contributing to the southeasterly groundwater flow direction would be potential mounding of groundwater 1 mi north of Canada due to increased recharge from Marion Lake (Figure 2.1; Appendix B).

\subsection{Grain Storage Operations and Property History}

Grain storage operations at Canada have changed considerably since the CCC/USDA facility was in operation, in 1954 to 1974 (Figures 2.3, 2.4, and 2.5). Harry Helmer of Hillsboro, Kansas, purchased the CCC/USDA bins in 1974 and removed them to begin a private grain storage business elsewhere (FSA 1997). Subsequently, the co-op expanded southward and installed the storage structures now located on the former CCC/USDA property. Other co-op grain storage structures (now removed) were north of the former CCC/USDA facility. Understanding grain storage operations at Canada before and after the CCC/USDA's period of operation (1954-1974) is critical to evaluating the potential source areas for the carbon tetrachloride contamination. The available leases for the former CCC/USDA property are reproduced in Appendix C.

In 1954, to supplement the co-op's available grain storage capacity to the north, the CCC/USDA leased approximately 1 acre of land in the southern portion of the town from Aaron Burkholder. The leased property included Lots 7-18 on Block 11 (Figure 2.2; Figure C.1 in Appendix C). Fifteen circular steel bins with cement floors were erected (Figure 2.3). Each bin had a storage capacity of 3,200 bushels, for a total storage capacity of 48,000 bushels. This initial $1954 \mathrm{CCC} / \mathrm{USDA}$ lease of the property was for a period of ten years. In 1964 the lease was extended until 1974 (Figure C.2 in Appendix C).

In 1965, early in the second lease period, Burkholder sold the leased property on which the CCC/USDA was operating its facility to Canada Grain Company of Marion, Kansas (Figure C.3 in Appendix C). In the absence of a lease termination agreement (which has not been found), the CCC/USDA is assumed to have continued its operation on the leased property until 1974, when Harry Helmer purchased and removed the CCC/USDA bins. 
With its 1965 purchase from Burkholder, the property owned by Canada Grain Company expanded to include the following (Figure 2.2):

- Block 3: Lots 1-6

- Block 4: Lots 1-6

- Block 6: Lots 1-2, Lots 7-12, the western half of Lots 13-15, Lots 16-18

- Block 11: Lots 7-18 (the former CCC/USDA facility)

Except for the eastern half of Lots 13-15 on Block 6, occupied by the Mueller residence garage, this land sale gave the Canada Grain Company title to the central portion of Canada, from Third Street north to the Atchison, Topeka, and Santa Fe Railroad spur. The capacity of the grain storage facility currently at this location is 416,811 bushels (CGS 2007).

Ownership of the grain storage operation changed several times in the years that followed. Ownership history for the property cited by the KDHE as part of its pre-CERCLIS site reconnaissance and evaluation (KDHE 1998) includes the following:

- Canada Grain — 1965-1977 (Figure C.3 in Appendix C)

- Cooperative Grain and Supply, Hillsboro - January 1977-May 1978 (Figure C.4 in Appendix C; property transferred included the former CCC/USDA facility)

- D\&R Trucking/D\&R Grain, Hillsboro - May 1978-October 1978 (Figure C.5 in Appendix C)

- Countryside Grain - October 1978-September 1985 (Figure C.5 in Appendix C)

- Daniel P. Janzen/Janzen Farm Service - September 1985-January 1991 (Figure C.6 in Appendix C) 
- Cooperative Grain and Supply, Hillsboro - 1991-present (Figure C.6 in Appendix C)

Aerial photos showing the changing grain storage structures over the decades are as follows:

- A 1956 aerial photo (Figure 2.3) shows the former CCC/USDA grain storage facility as consisting of 15 circular bins in the southern portion of town. Large storage structures of unknown use are visible on the co-op property, just north of the former CCC/USDA facility. Wooden grain elevators are visible in the northern portion of the co-op property, immediately south of the former railroad.

- A 1991 aerial photo (Figure 2.4) shows co-op structures on the former CCC/USDA property (which the CCC/USDA vacated in 1974). Changes are also evident in 1991 (Figure 2.4) on the central portion of the co-op property. Two large storage structures of unknown use appearing just north of the former CCC/USDA property in 1956 (peach highlights in Figure 2.4) are absent in 1991 (compare to gray highlights in Figure 2.4). One circular storage bin and one circular bin foundation are visible in the 1991 photo (gray highlights in Figure 2.4) on the co-op property, just north of the former CCC/USDA property. The foundation of a flat storage building is seen farther north. The door of the flat storage building appears to have been at the southern end of the building. The photographs in Figures 2.3 and 2.4 demonstrate that this flat storage building was installed after 1956 and removed before 1991 .

- The 2002 aerial photograph (Figure 2.5) shows the current configuration of the grain storage operations at Canada. Most of the structures visible in the 1991 photograph (Figure 2.4) remain. 


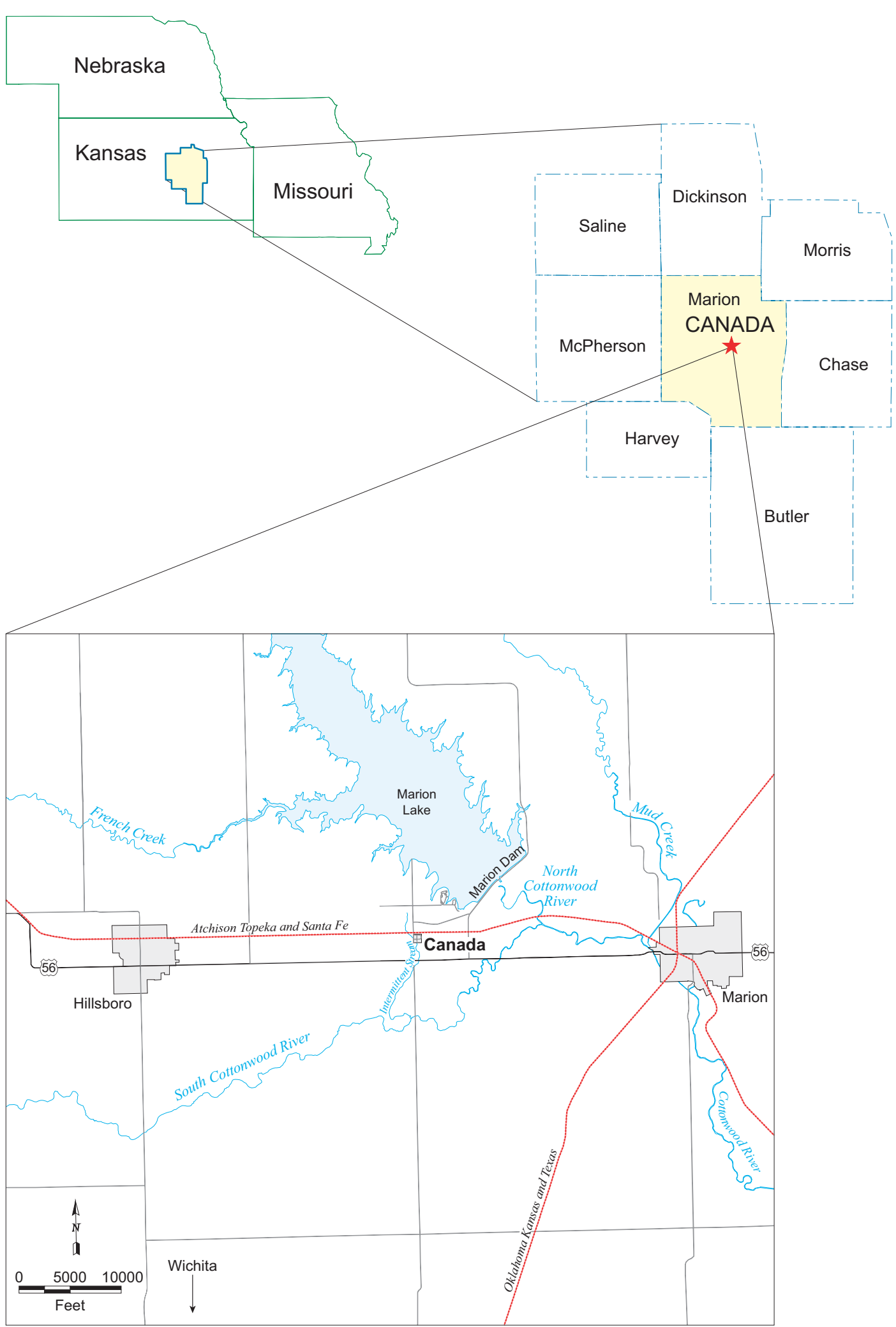

FIGURE 2.1 Location of Canada, Kansas. 


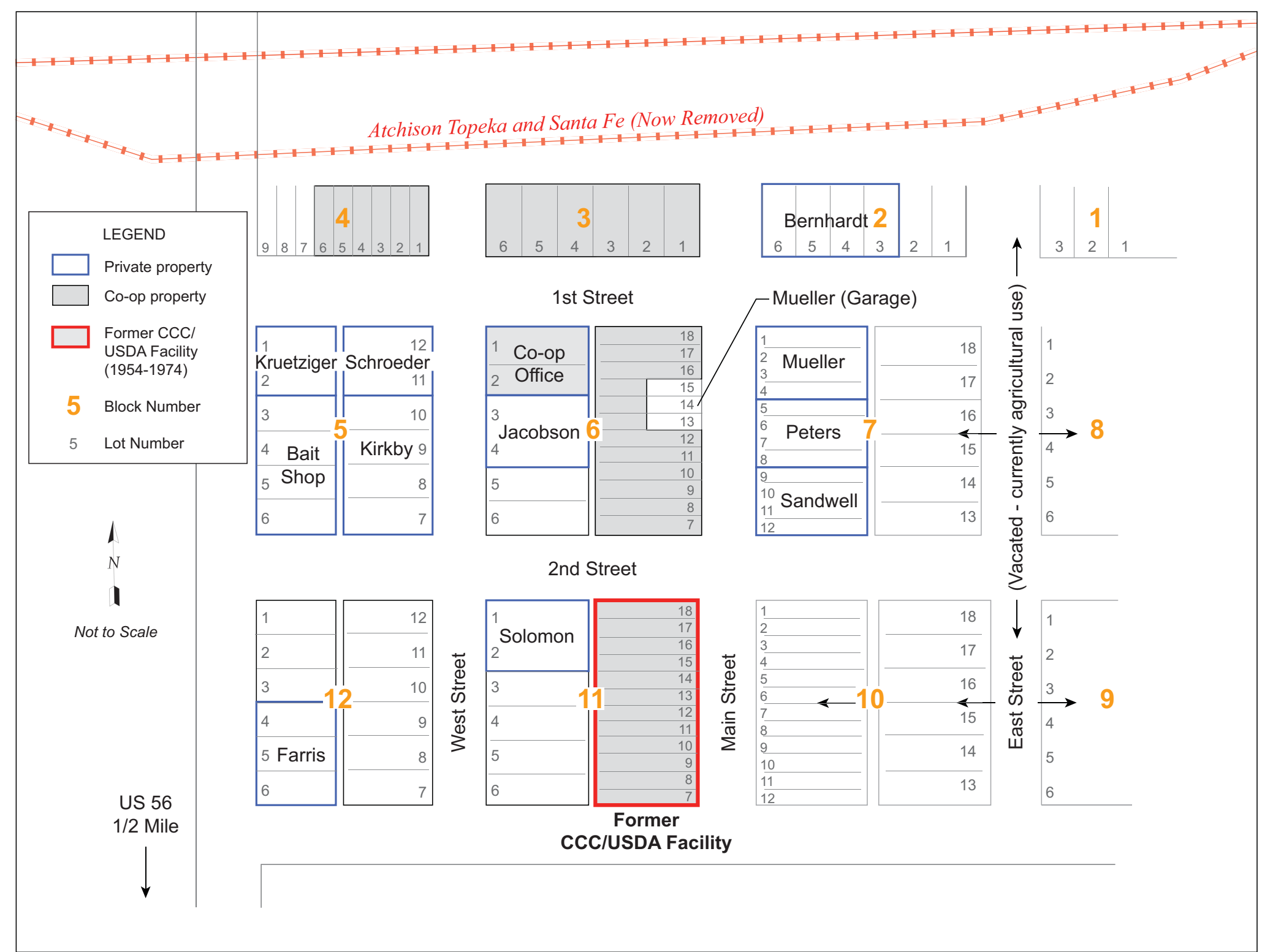

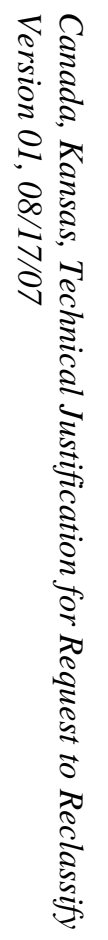

FIGURE 2.2 Plat of Canada, Kansas. Sources of data: State of Kansas (1883), KDHE (1998). 


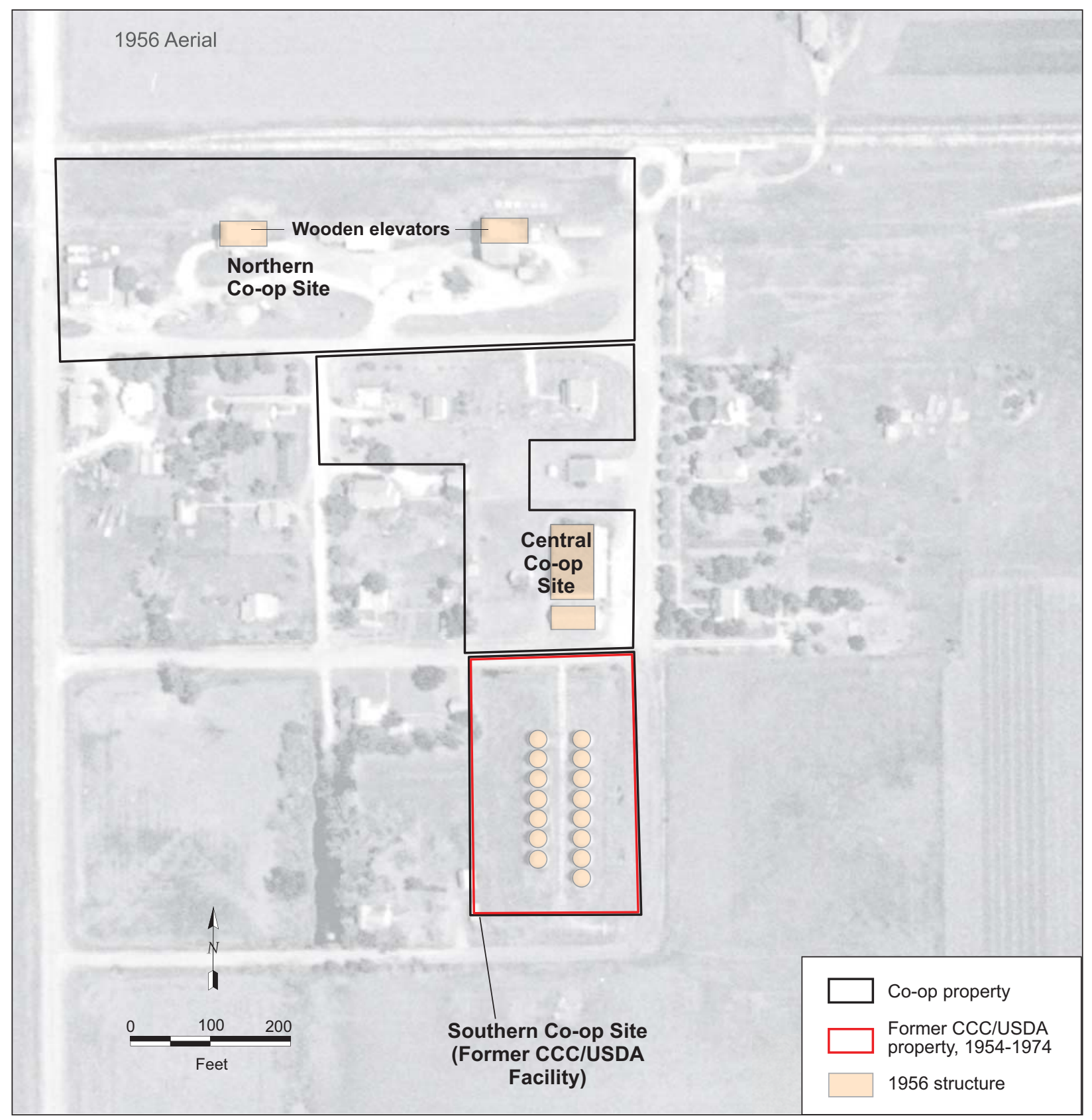

FIGURE 2.3 Grain storage structures at Canada in 1956, with property boundaries. Source of photograph: USDA (1956). 


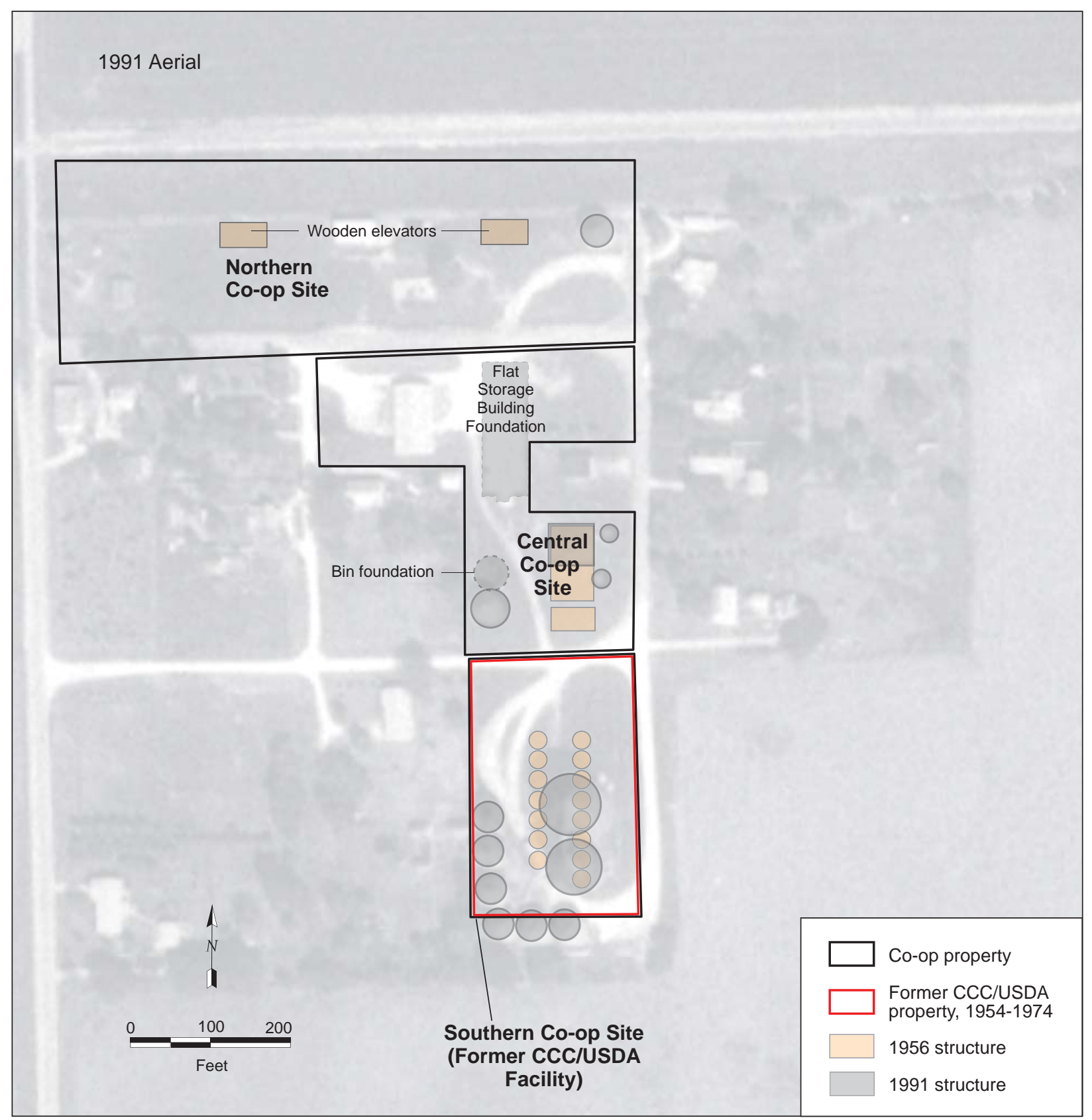

FIGURE 2.4 Grain storage structures at Canada in 1991, with locations of 1956 structures shown in Figure 2.3 and property boundaries. Source of photograph: USGS (1991). 


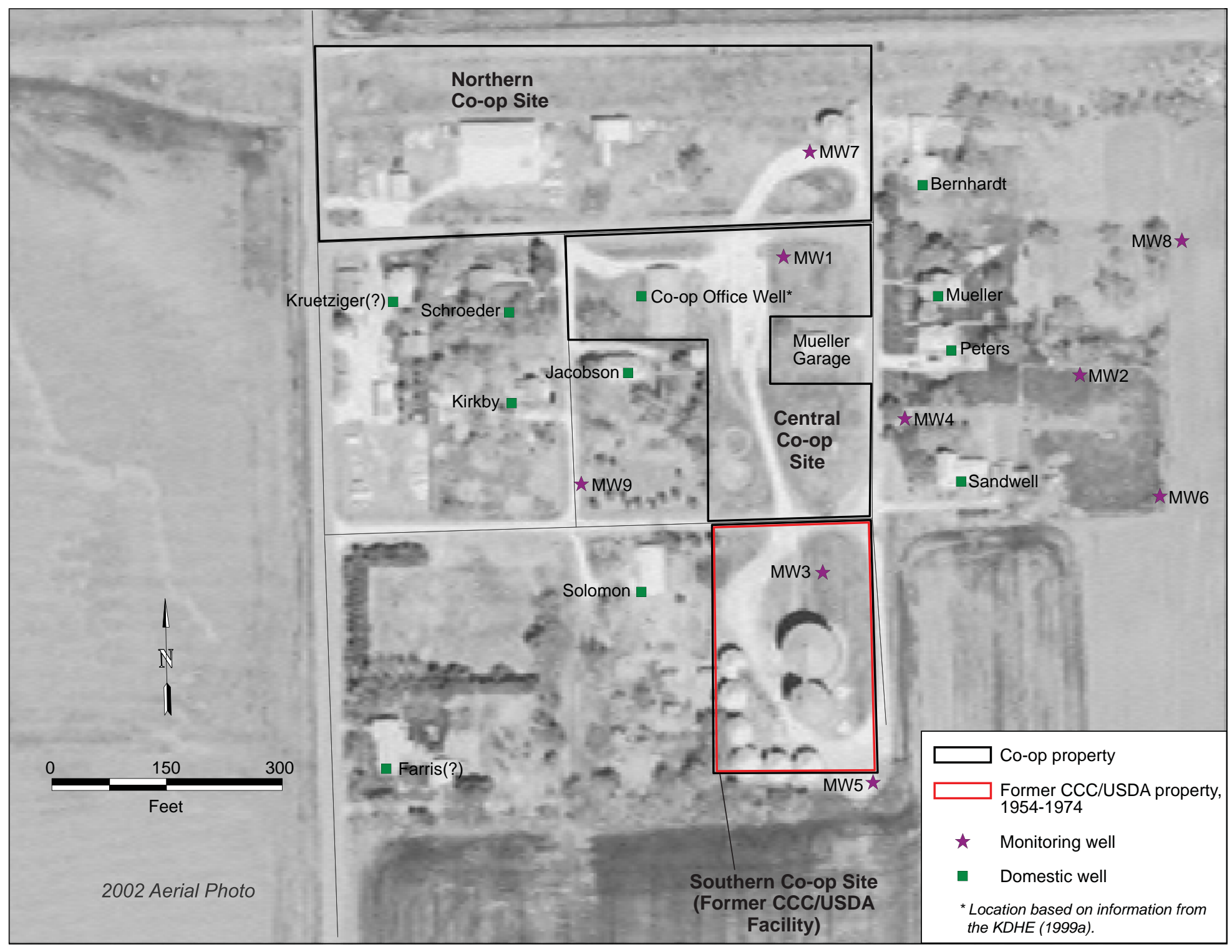

FIGURE 2.5 Current configuration of the Canada site. Source of photograph: USDA (2002). 


\section{Carbon Tetrachloride Contamination at Canada}

\subsection{Identification of Contamination and Removal Action}

Carbon tetrachloride contamination was initially identified at Canada in October 1997 (KDHE 1997), in conjunction with the private well sampling program conducted by the KDHE at former CCC/USDA facilities. Contamination was detected in two of the four wells sampled $(24.2 \mu \mathrm{g} / \mathrm{L}$ in the Mueller private well and $1.1 \mu \mathrm{g} / \mathrm{L}$ in the Cooperative Grain and Supply well; Table 3.1). In subsequent KDHE investigations, including a site reconnaissance and evaluation in 1997-1998 (KDHE 1998) and a preliminary assessment/screening site inspection in 1999 (KDHE 1999a), all private wells in Canada were sampled.

The Mueller well has continued to exhibit carbon tetrachloride at levels above the MCL of $5.0 \mu \mathrm{g} / \mathrm{L}$. The Peters wells has also contained levels of carbon tetrachloride near and above the MCL. Both the Mueller and Peters wells are to the east of the co-op property. The results of the most recent sampling are in Figure 3.1.

In addition to the carbon tetrachloride contamination, pervasive nitrate contamination above the MCL of $10 \mathrm{mg} / \mathrm{L}$ has been present (Table 3.1). The nitrate contamination is not associated with activities of the CCC/USDA.

All residents of Canada formerly obtained their water from private wells. On the basis of the 1997-1999 KDHE results, the CCC/USDA instituted two removal actions to provide affected residents with alternate water supplies:

- The CCC/USDA provided a temporary alternate water supply of bottled water to the Mueller and Peters residences after the 1998 site reconnaissance and evaluation (KDHE 1999a). This action was in response to the detection of carbon tetrachloride in these residences' wells at concentrations above the MCL of $5.0 \mu \mathrm{g} / \mathrm{L}$ for drinking water.

- The Marion RWD \#4 line was extended into Canada as a permanent wholehouse alternate water supply in April 1999. The CCC/USDA provided funding to connect the Mueller residence to the RWD supply line (Shewey 
1999). Mr. Peters elected to pay for the connection of his residence to the RWD water supply line.

The KDHE concluded in its preliminary removal site evaluation (KDHE 1999b) that no further removal site evaluation, removal response, or removal action in Canada was warranted, in view of the provision of the permanent alternate water supply.

\subsection{Contaminant History and Previous Investigations}

In 1999, the KDHE $(1999 \mathrm{a}, \mathrm{c})$ conducted soil sampling to identify the source of the carbon tetrachloride contamination at Canada. Figure 3.2 shows the soil sample locations, plotted by using ( $\mathrm{x}, \mathrm{y})$ measurements reported by the KDHE (1999a).

Samples collected at four of seven locations north of the former CCC/USDA bins contained carbon tetrachloride at $0.2 \mu \mathrm{g} / \mathrm{kg}$ (at location SP7), $0.3 \mu \mathrm{g} / \mathrm{kg}$ (at SP4 and SP8), and $0.9 \mu \mathrm{g} / \mathrm{kg}$ (at SP12) (Figure 3.2). Carbon tetrachloride was not detected at SP3, SP9, and SP10, also north of the former CCC/USDA bins. In contrast, carbon tetrachloride was found in only one of five soil samples collected between and adjacent to the former CCC/USDA bins $(0.2 \mu \mathrm{g} / \mathrm{kg}$ at SP6); the contaminant was not detected in samples from locations SP1, SP2, SP5, and SP11, in this same area. This finding is significant, because contamination resulting from CCC/USDA activities would be most likely in the doorways and around foundations of CCC/USDA bins. Contamination elsewhere on the former CCC/USDA property could have resulted from either CCC/USDA or co-op activities. The co-op has occupied the property since at least 1974.

During a comprehensive investigation completed in 2001 (KDHE 2001), the KDHE installed nine monitoring wells. Since that time, the KDHE has conducted long-term monitoring of these wells and four private wells. A chronological summary of the analytical results for groundwater sampling at Canada is in Table 3.1. The carbon tetrachloride results for the KDHE's 2007 monitoring are displayed in Figure 3.1.

The KDHE's long-term water level measurements (Table 3.2) and reported survey coordinates (Table 3.3) were used to generate the maps of groundwater flow direction in 
Figures 3.3-3.6. These figures indicate a consistent groundwater flow direction to the east or east-southeast during the 2001-2007 monitoring, though the water levels have fluctuated.

Table 3.4 shows the historical analytical results for carbon tetrachloride in individual wells. Carbon tetrachloride levels in the Mueller and Peters private wells have generally declined since 1997 (from $24.2 \mu \mathrm{g} / \mathrm{L}$ to $9.0 \mu \mathrm{g} / \mathrm{L}$ [2007] in the Mueller well and from $12.7 \mu \mathrm{g} / \mathrm{L}$ to $2.8 \mu \mathrm{g} / \mathrm{L}$ [2005] in the Peters well). In contrast, the absence of a declining trend in contaminant levels in MW2 (with values of 10.0-22.0 $\mu \mathrm{g} / \mathrm{L}$; Table 3.4) in 2001-2007 suggests an ongoing source in the vicinity of the co-op's former flat storage building, upgradient from this well (Figure 3.3). For comparison, the level of carbon tetrachloride in MW3, at the north end of the former CCC/USDA facility (east-southeast of the two circular co-op storage structures visible on the 1991 aerial photo in Figure 3.3) has varied between $0.63 \mu \mathrm{g} / \mathrm{L}$ and $2.9 \mu \mathrm{g} / \mathrm{L}$ in $2001-2007$.

The fumigant compounds 1,2-dichloroethane, ethylene dibromide, and tetrachloroethylene have been detected at trace concentrations (Table 3.4) at monitoring locations MW4 (downgradient from the co-op's former flat storage building) and MW7 (downgradient from the former wooden elevators at the north end of the co-op property). These compounds have not been detected in monitoring wells MW3 and MW5, located on and downgradient from the former CCC/USDA property, respectively.

Fuel-related compounds (benzene, 1,1-dichloroethane [DCA], ethylbenzene, methyl tertbutyl ether [MTBE], sec-butylbenzene, trimethylbenzene, xylene) were identified at low concentrations in the Sandwell private well in 1998 and in monitoring wells MW1, MW4, and MW8 in 2003 and 2005 (Table 3.4). These wells lie east (downgradient) of the co-op (Figure 2.5). A documented release of diesel fuel occurred on May 28, 1991, on the northeast corner of the co-op (at that time the Janzen Farm Service), when a ten-year-old, 9,000-gal underground storage tank was being removed. A slight odor of diesel fuel was evident on a lower sidewall of the tank excavation, but no staining was observed. Contaminated soil was removed for aeration (KDHE 2007b).

The KDHE noted in an internal memorandum that the fuel-related compounds discussed above, plus ethylene dibromide [EDB], had been found in wells downgradient from the co-op. The memorandum (KDHE 2004) stated the following: "Benzene, DCA, sec-butylbenzene, MTBE, and EDB are contaminants associated with gasoline releases. EDB is also used as a grain fumigant. A closed [leaking underground storage tank] site is located within the subject area 
[where long-term monitoring is being conducted] and EDB was detected downgradient of current and former grain storage locations; however, reported impacts associated with these contaminants appear to be highly localized, do not affect private wells, and do not exceed MCLs." The 2003 results for ethylene dibromide to which the KDHE memorandum referred are in Table 3.4. None of the compounds mentioned have been detected in monitoring wells MW3 and MW5, located on and downgradient from the former CCC/USDA property, respectively. 
TABLE 3.1 Chronological history of groundwater sampling and analysis at Canada. ${ }^{a}$

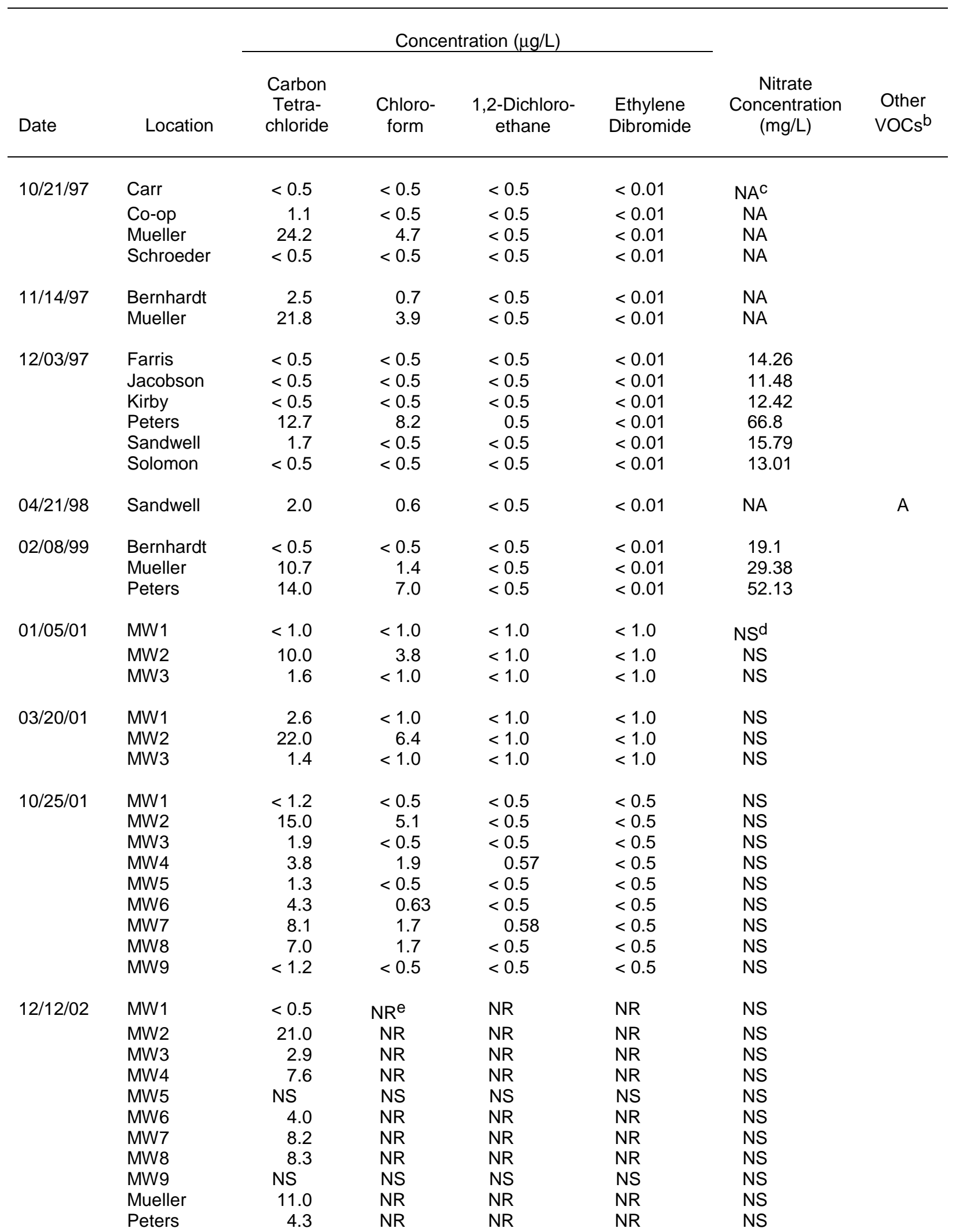


TABLE 3.1 (Cont.)

\begin{tabular}{|c|c|c|c|c|c|c|c|}
\hline \multirow[b]{2}{*}{ Date } & \multirow[b]{2}{*}{ Location } & \multicolumn{4}{|c|}{ Concentration $(\mu \mathrm{g} / \mathrm{L})$} & \multirow[b]{2}{*}{$\begin{array}{c}\text { Nitrate } \\
\text { Concentration } \\
(\mathrm{mg} / \mathrm{L})\end{array}$} & \multirow[b]{2}{*}{$\begin{array}{l}\text { Other } \\
\text { VOCs }\end{array}$} \\
\hline & & $\begin{array}{c}\text { Carbon } \\
\text { Tetra- } \\
\text { chloride }\end{array}$ & $\begin{array}{l}\text { Chloro- } \\
\text { form }\end{array}$ & $\begin{array}{l}\text { 1,2-Dichloro- } \\
\text { ethane }\end{array}$ & $\begin{array}{l}\text { Ethylene } \\
\text { Dibromide }\end{array}$ & & \\
\hline \multirow[t]{13}{*}{$12 / 29 / 03$} & MW1 & $<0.5$ & $<0.5$ & $<0.5$ & $<0.01$ & NS & B \\
\hline & MW2 & 15.0 & 5.5 & $<0.5$ & $<0.01$ & NS & $\mathrm{C}$ \\
\hline & MW3 & 1.9 & $<0.5$ & $<0.5$ & $<0.01$ & NS & \\
\hline & MW4 & 4.4 & 4.3 & 2.2 & $<0.01$ & NS & D \\
\hline & MW5 & 1.3 & $<0.5$ & $<0.5$ & $<0.01$ & NS & \\
\hline & MW6 & 3.7 & $<0.5$ & $<0.5$ & $<0.01$ & NS & E \\
\hline & MW7 & 8.0 & 1.4 & $<0.5$ & 0.022 & NS & $\mathrm{F}$ \\
\hline & MW8 & 6.9 & 1.5 & $<0.5$ & $<0.01$ & NS & G \\
\hline & MW9 & NS & NS & NS & NS & NS & \\
\hline & Bernhardt & 2.0 & $<0.5$ & $<0.5$ & $<0.01$ & 12.0 & $\mathrm{H}$ \\
\hline & Mueller & 10.0 & 1.0 & $<0.5$ & $<0.01$ & 19.0 & \\
\hline & Peters & 4.7 & 0.84 & $<0.5$ & $<0.01$ & 20.0 & \\
\hline & Sandwell & 1.1 & $<0.5$ & $<0.5$ & $<0.01$ & 16.0 & \\
\hline \multirow[t]{13}{*}{$12 / 02 / 05$} & MW1 & 1.0 & $<0.5$ & $<0.5$ & $<0.01$ & NS & \\
\hline & MW2 & 13.0 & 4.4 & $<0.5$ & $<0.01$ & NS & \\
\hline & MW3 & 0.63 & $<0.5$ & $<0.5$ & $<0.01$ & NS & \\
\hline & MW4 & 5.6 & 2.0 & 1.4 & $<0.01$ & NS & I \\
\hline & MW5 & NS & NS & NS & NS & NS & \\
\hline & MW6 & 2.1 & $<0.5$ & $<0.5$ & $<0.01$ & NS & \\
\hline & MW7 & 4.0 & 0.92 & $<0.5$ & 0.022 & NS & \\
\hline & MW8 & 4.4 & 1.4 & $<0.5$ & $<0.01$ & NS & $J$ \\
\hline & MW9 & NS & NS & NS & NS & NS & \\
\hline & Bernhardt & NS & NS & NS & NS & NS & \\
\hline & Mueller & 16.0 & 3.2 & $<0.5$ & $<0.01$ & 28 & \\
\hline & Peters & 2.8 & 1.0 & $<0.5$ & $<0.01$ & 22.0 & \\
\hline & Sandwell & 1.9 & 0.57 & $<0.5$ & $<0.01$ & 13.0 & \\
\hline \multirow{13}{*}{$02 / 20 / 07$} & MW1 & $<0.5$ & $<0.5$ & $<0.5$ & $<0.01$ & NS & \\
\hline & MW2 & 18 & 6.4 & 0.62 & $<0.01$ & NS & \\
\hline & MW3 & 1.1 & $<0.5$ & $<0.5$ & $<0.01$ & NS & \\
\hline & MW4 & 2.8 & 1.1 & 1.6 & $<0.01$ & NS & \\
\hline & MW5 & NS & NS & NS & NS & NS & \\
\hline & MW6 & 4.7 & 0.62 & $<0.5$ & $<0.01$ & NS & \\
\hline & MW7 & 6.2 & 1.0 & $<0.5$ & $<0.01$ & NS & \\
\hline & MW8 & 6.3 & 1.5 & $<0.5$ & $<0.01$ & NS & \\
\hline & MW9 & NS & NS & NS & NS & NS & \\
\hline & Bernhardt & 2.1 & 0.71 & $<0.5$ & $<0.01$ & 12 & $\mathrm{~K}$ \\
\hline & Mueller & 9.0 & 1.9 & $<0.5$ & $<0.01$ & 20 & \\
\hline & Peters & NS & NS & NS & NS & NS & \\
\hline & Sandwell & 0.6 & $<0.5$ & $<0.5$ & $<0.01$ & 16 & \\
\hline
\end{tabular}




\section{TABLE 3.1 (Cont.)}

a Results are from off-site laboratories. Sources of data: Dallen 1998, 1999; KDHE 1997, 1998, 1999a, 2001, 2004, 2006, 2007a.

b Codes for other volatile organic compounds (concentrations in $\mu \mathrm{g} / \mathrm{L}$ ):

A: $04 / 21 / 98$, Sandwell: ethylbenzene $=1.0 ;$ xylene $=3.9 ; 1,3,5$-trimethylbenzene $=0.5$; 1,2,4-trimethylbenzene $=0.7$.

B: $12 / 29 / 03$, MW1: chloromethane $=12$; sec-butylbenzene $=8.3$; methyl tert-butyl ether $=6.4$.

C: $12 / 29 / 03$, MW2: chloromethane $=5.8 ; 1,1$-dichloroethane $=0.75$.

D: $12 / 29 / 03$, MW4: benzene $=1.7$; tetrachloroethylene $=0.57$; chloromethane $=4.3$.

E: $12 / 29 / 03$, MW6: chloromethane $=1.6$.

F: $12 / 29 / 03$, MW7: chloromethane $=14$.

G: $12 / 29 / 03$, MW8: chloromethane $=7.5$.

$\mathrm{H}: \quad 12 / 29 / 03$, Bernhardt: chloromethane $=0.72$.

I: $12 / 02 / 05$, MW4: benzene $=0.51$.

J: $12 / 02 / 05$, MW8: 1,1-dichloroethane $=0.52$.

$\mathrm{K}: \quad 02 / 20 / 07$, Bernhardt: chloromethane $=0.71$.

c NA, not analyzed for this constituent.

d NS, monitoring location not sampled for this constituent in this event.

e NR, result not reported in the investigation reports on file. 
TABLE 3.2 Water Level data for the Canada site. Source of data: $\operatorname{KDHE}(2001,2004,2006,2007 a)$.

\begin{tabular}{|c|c|c|c|c|c|c|c|c|c|}
\hline \multirow[b]{3}{*}{ Well } & \multirow{3}{*}{$\begin{array}{c}\text { Top of Casing } \\
\text { Elevation } \\
\text { (ft AMSL) }\end{array}$} & \multicolumn{8}{|c|}{ Water Surface Depth or Elevation in Year Indicated } \\
\hline & & \multicolumn{2}{|c|}{2007} & \multicolumn{2}{|c|}{2005} & \multicolumn{2}{|c|}{2003} & \multicolumn{2}{|c|}{2001} \\
\hline & & $\begin{array}{l}\text { Depth (ft } \\
\text { BGL) }\end{array}$ & $\begin{array}{l}\text { Elevation } \\
\text { (ft AMSL) }\end{array}$ & $\begin{array}{l}\text { Depth (ft } \\
\text { BGL) }\end{array}$ & $\begin{array}{c}\text { Elevation (ft } \\
\text { AMSL) }\end{array}$ & $\begin{array}{c}\text { Depth } \\
\text { (ft BGL) }\end{array}$ & $\begin{array}{c}\text { Elevation (ft } \\
\text { AMSL) }\end{array}$ & $\begin{array}{l}\text { Depth (ft } \\
\text { BGL) }\end{array}$ & $\begin{array}{l}\text { Elevation } \\
\text { (ft AMSL) }\end{array}$ \\
\hline MW1 & 1363.05 & 20.85 & 1342.2 & 15.34 & 1347.71 & 17.71 & 1345.34 & 17.10 & 1345.95 \\
\hline MW2 & 1361.55 & 19.71 & 1341.84 & 14.74 & 1346.81 & 16.84 & 1344.71 & 16.18 & 1345.37 \\
\hline MW3 & 1360.33 & 18.13 & 1342.2 & 12.14 & 1348.19 & 15.04 & 1345.29 & 14.31 & 1346.02 \\
\hline MW4 & 1362.44 & 20.45 & 1341.99 & 15.28 & 1347.16 & 17.55 & 1344.89 & 16.88 & 1345.56 \\
\hline MW5 & 1360.65 & $N S^{a}$ & NS & NSa & & 15.33 & 1345.32 & 14.65 & 1346.00 \\
\hline MW6 & 1360.32 & 18.5 & 1341.82 & 13.65 & 1346.67 & 15.65 & 1344.67 & 15.00 & 1345.32 \\
\hline MW7 & 1364.54 & 21.55 & 1342.99 & 16.36 & 1348.18 & 18.44 & 1346.10 & 17.87 & 1346.67 \\
\hline MW8 & 1361.01 & 19.2 & 1341.81 & 14.30 & 1346.71 & 16.36 & 1344.65 & 15.67 & 1345.34 \\
\hline MW9 & 1358.01 & NS & NS & NS & & NS & & 11.00 & 1347.01 \\
\hline
\end{tabular}

a NS, not sampled. 
TABLE 3.3 Survey data for the Canada site. Source of data:

KDHE (2001).

\begin{tabular}{lllll}
\hline Location & North & West & & \\
& & & & \\
& & & & \\
Latitude & & \\
LW1 & 2348.84 & 4707.25 & $38-21-17$ & $97-06-42$ \\
MW2 & 2235.35 & 4306.89 & $38-21-15$ & $97-06-38$ \\
MW3 & 1953.59 & 4665.80 & $38-21-12$ & $97-06-41$ \\
MW4 & 2174.83 & 4575.79 & $38-21-15$ & $97-06-39$ \\
MW5 & 1659.27 & 4601.71 & $38-21-10$ & $97-06-39$ \\
MW6 & 2035.95 & 4239.72 & $38-21-14$ & $97-06-36$ \\
MW7 & 2509.07 & 4672.34 & $38-21-18$ & $97-06-41$ \\
MW8 & 2400.49 & 4207.03 & $38-21-16$ & $97-06-36$ \\
MW9 & 2059.19 & 4971.87 & $38-21-15$ & $97-06-44$ \\
\hline
\end{tabular}

a The Canada site is located in Section 33, Township 19 South, Range 3 East of the Sixth Principal Meridian, Marion County, Kansas. The southeast corner of Section 33 was assigned coordinates of 00.00 North and 00.00 West.

b Latitude and longitude were scaled from a 7.5-minute quadrangle map (USGS 1985). 
TABLE 3.4 Historical results of groundwater sampling and analysis for individual wells at Canada. ${ }^{a}$

\begin{tabular}{|c|c|c|c|c|c|c|c|}
\hline \multirow[b]{2}{*}{ Location } & \multirow[b]{2}{*}{ Date } & \multicolumn{4}{|c|}{ Concentration $(\mu \mathrm{g} / \mathrm{L})$} & \multirow[b]{2}{*}{$\begin{array}{c}\text { Nitrate } \\
\text { Concentration } \\
(\mathrm{mg} / \mathrm{L})\end{array}$} & \multirow[b]{2}{*}{$\begin{array}{l}\text { Other } \\
\text { VOCs }^{b}\end{array}$} \\
\hline & & $\begin{array}{l}\text { Carbon } \\
\text { Tetra- } \\
\text { chloride }\end{array}$ & $\begin{array}{l}\text { Chloro- } \\
\text { form }\end{array}$ & $\begin{array}{l}\text { 1,2-Dichloro- } \\
\text { ethane }\end{array}$ & $\begin{array}{l}\text { Ethylene } \\
\text { Dibromide }\end{array}$ & & \\
\hline \multirow[t]{3}{*}{ Bernhardt } & $11 / 14 / 97$ & 2.5 & 0.7 & $<0.5$ & $<0.01$ & $\mathrm{NA}^{\mathrm{C}}$ & \\
\hline & $\begin{array}{l}02 / 08 / 99 \\
12 / 29 / 03 \\
12 / 02 / 05\end{array}$ & $\begin{array}{r}<0.5 \\
2.0 \\
\text { NSd }^{d}\end{array}$ & $\begin{array}{l}<0.5 \\
<0.5 \\
\text { NS }\end{array}$ & $\begin{array}{l}<0.5 \\
<0.5 \\
\text { NS }\end{array}$ & $\begin{array}{l}<0.01 \\
<0.01 \\
\text { NS }\end{array}$ & $\begin{array}{l}19.1 \\
12.0 \\
\text { NS }\end{array}$ & A \\
\hline & 02/20/07 & 2.1 & 0.71 & $<0.5$ & $<0.01$ & 12 & $B$ \\
\hline Carr & $10 / 21 / 97$ & $<0.5$ & $<0.5$ & $<0.5$ & $<0.01$ & NA & \\
\hline Co-op & $10 / 21 / 97$ & 1.1 & $<0.5$ & $<0.5$ & $<0.01$ & NA & \\
\hline Farris & $12 / 03 / 97$ & $<0.5$ & $<0.5$ & $<0.5$ & $<0.01$ & 14.26 & \\
\hline Jacobson & $12 / 03 / 97$ & $<0.5$ & $<0.5$ & $<0.5$ & $<0.01$ & 11.48 & \\
\hline Kirby & $12 / 03 / 97$ & $<0.5$ & $<0.5$ & $<0.5$ & $<0.01$ & 12.42 & \\
\hline \multirow[t]{2}{*}{ Mueller } & $\begin{array}{l}10 / 21 / 97 \\
11 / 14 / 97 \\
02 / 08 / 99 \\
12 / 12 / 02\end{array}$ & $\begin{array}{l}24.2 \\
21.8 \\
10.7 \\
11.0\end{array}$ & $\begin{array}{r}4.7 \\
3.9 \\
1.4 \\
N R^{\mathrm{e}}\end{array}$ & $\begin{array}{l}<0.5 \\
<0.5 \\
<0.5 \\
\text { NR }\end{array}$ & $\begin{array}{l}<0.01 \\
<0.01 \\
<0.01 \\
\text { NR }\end{array}$ & $\begin{array}{l}\text { NA } \\
\text { NA } \\
29.38 \\
\text { NS }\end{array}$ & \\
\hline & $\begin{array}{l}12 / 29 / 03 \\
12 / 02 / 05 \\
02 / 20 / 07\end{array}$ & $\begin{array}{r}10.0 \\
16.0 \\
9.0\end{array}$ & $\begin{array}{l}1.0 \\
3.2 \\
1.9\end{array}$ & $\begin{array}{l}<0.5 \\
<0.5 \\
<0.5\end{array}$ & $\begin{array}{l}<0.01 \\
<0.01 \\
<0.01\end{array}$ & $\begin{array}{l}19.0 \\
28 \\
20\end{array}$ & \\
\hline Peters & $\begin{array}{l}12 / 03 / 97 \\
02 / 08 / 99 \\
12 / 12 / 02 \\
12 / 29 / 03 \\
12 / 02 / 05 \\
02 / 20 / 07\end{array}$ & $\begin{array}{r}12.7 \\
14.0 \\
4.3 \\
4.7 \\
2.8 \\
\text { NS }\end{array}$ & $\begin{array}{c}8.2 \\
7.0 \\
\text { NR } \\
0.84 \\
1.0 \\
\text { NS }\end{array}$ & $\begin{aligned} & 0.5 \\
&< 0.5 \\
& \text { NR } \\
&< 0.5 \\
&< 0.5 \\
& \text { NS }\end{aligned}$ & $\begin{array}{l}<0.01 \\
<0.01 \\
\text { NR } \\
<0.01 \\
<0.01 \\
\text { NS }\end{array}$ & $\begin{array}{l}66.8 \\
52.13 \\
\text { NS } \\
20.0 \\
22.0 \\
\text { NS }\end{array}$ & \\
\hline Sandwell & $\begin{array}{l}12 / 03 / 97 \\
04 / 21 / 98 \\
12 / 29 / 03 \\
12 / 02 / 05 \\
02 / 20 / 07\end{array}$ & $\begin{array}{l}1.7 \\
2.0 \\
1.1 \\
1.9 \\
0.6\end{array}$ & $\begin{aligned}< & 0.5 \\
& 0.6 \\
< & 0.5 \\
& 0.57 \\
< & 0.5\end{aligned}$ & $\begin{array}{l}<0.5 \\
<0.5 \\
<0.5 \\
<0.5 \\
<0.5\end{array}$ & $\begin{array}{l}<0.01 \\
<0.01 \\
<0.01 \\
<0.01 \\
<0.01\end{array}$ & $\begin{array}{l}15.79 \\
\text { NA } \\
16.0 \\
13.0 \\
16\end{array}$ & C \\
\hline Schroeder & $10 / 21 / 97$ & $<0.5$ & $<0.5$ & $<0.5$ & $<0.01$ & NA & \\
\hline Solomon & $12 / 03 / 97$ & $<0.5$ & $<0.5$ & $<0.5$ & $<0.01$ & 13.01 & \\
\hline MW1 & $\begin{array}{l}01 / 05 / 01 \\
03 / 20 / 01 \\
10 / 25 / 01 \\
12 / 12 / 02 \\
12 / 29 / 03 \\
12 / 02 / 05 \\
02 / 20 / 07\end{array}$ & $\begin{aligned}< & 1.0 \\
& 2.6 \\
< & 1.2 \\
< & 0.5 \\
< & 0.5 \\
& 1.0 \\
< & 0.5\end{aligned}$ & $\begin{array}{l}<1.0 \\
<1.0 \\
<0.5 \\
\text { NR } \\
<0.5 \\
<0.5 \\
<0.5\end{array}$ & $\begin{array}{l}<1.0 \\
<1.0 \\
<0.5 \\
\text { NR } \\
<0.5 \\
<0.5 \\
<0.5\end{array}$ & $\begin{array}{l}<1.0 \\
<1.0 \\
<0.5 \\
\text { NR } \\
<0.01 \\
<0.01 \\
<0.01\end{array}$ & $\begin{array}{l}\text { NS } \\
\text { NS } \\
\text { NS } \\
\text { NS } \\
\text { NS } \\
\text { NS } \\
\text { NS }\end{array}$ & $D$ \\
\hline
\end{tabular}


TABLE 3.4 (Cont.)

\begin{tabular}{|c|c|c|c|c|c|c|c|}
\hline \multirow[b]{2}{*}{ Location } & \multirow[b]{2}{*}{ Date } & \multicolumn{4}{|c|}{ Concentration $(\mu \mathrm{g} / \mathrm{L})$} & \multirow[b]{2}{*}{$\begin{array}{c}\text { Nitrate } \\
\text { Concentration } \\
(\mathrm{mg} / \mathrm{L})\end{array}$} & \multirow[b]{2}{*}{$\begin{array}{c}\text { Other } \\
\text { VOCs }^{b}\end{array}$} \\
\hline & & $\begin{array}{l}\text { Carbon } \\
\text { Tetra- } \\
\text { chloride }\end{array}$ & $\begin{array}{l}\text { Chloro- } \\
\text { form }\end{array}$ & $\begin{array}{l}\text { 1,2-Dichloro- } \\
\text { ethane }\end{array}$ & $\begin{array}{l}\text { Ethylene } \\
\text { Dibromide }\end{array}$ & & \\
\hline MW2 & $\begin{array}{l}01 / 05 / 01 \\
03 / 20 / 01 \\
10 / 25 / 01 \\
12 / 12 / 02 \\
12 / 29 / 03 \\
12 / 02 / 05 \\
02 / 20 / 07\end{array}$ & $\begin{array}{l}10.0 \\
22.0 \\
15.0 \\
21.0 \\
15.0 \\
13.0 \\
18.0\end{array}$ & $\begin{array}{c}3.8 \\
6.4 \\
5.1 \\
\text { NR } \\
5.5 \\
4.4 \\
6.4\end{array}$ & $\begin{aligned}< & 1.0 \\
< & 1.0 \\
< & 0.5 \\
\text { NR } & \\
< & 0.5 \\
< & 0.5 \\
& 0.62\end{aligned}$ & $\begin{array}{l}<1.0 \\
<1.0 \\
<0.5 \\
\text { NR } \\
<0.01 \\
<0.01 \\
<0.01\end{array}$ & $\begin{array}{l}\text { NS } \\
\text { NS } \\
\text { NS } \\
\text { NS } \\
\text { NS } \\
\text { NS } \\
\text { NS }\end{array}$ & $E$ \\
\hline MW3 & $\begin{array}{l}01 / 05 / 01 \\
03 / 20 / 01 \\
10 / 25 / 01 \\
12 / 12 / 02 \\
12 / 29 / 03 \\
12 / 02 / 05 \\
02 / 20 / 07\end{array}$ & $\begin{array}{l}1.6 \\
1.4 \\
1.9 \\
2.9 \\
1.9 \\
0.63 \\
1.1\end{array}$ & $\begin{array}{l}<1.0 \\
<1.0 \\
<0.5 \\
\text { NR } \\
<0.5 \\
<0.5 \\
<0.5\end{array}$ & $\begin{array}{l}<1.0 \\
<1.0 \\
<0.5 \\
\text { NR } \\
<0.5 \\
<0.5 \\
<0.5\end{array}$ & $\begin{array}{l}<1.0 \\
<1.0 \\
<0.5 \\
\text { NR } \\
<0.01 \\
<0.01 \\
<0.01\end{array}$ & $\begin{array}{l}\text { NS } \\
\text { NS } \\
\text { NS } \\
\text { NS } \\
\text { NS } \\
\text { NS } \\
\text { NS }\end{array}$ & \\
\hline MW4 & $\begin{array}{l}10 / 25 / 01 \\
12 / 12 / 02 \\
12 / 29 / 03 \\
12 / 02 / 05 \\
02 / 20 / 07\end{array}$ & $\begin{array}{l}3.8 \\
7.6 \\
4.4 \\
5.6 \\
2.8\end{array}$ & $\begin{array}{c}1.9 \\
\text { NR } \\
4.3 \\
2.0 \\
1.1\end{array}$ & $\begin{array}{c}0.57 \\
\text { NR } \\
2.2 \\
1.4 \\
1.6\end{array}$ & $\begin{array}{l}<0.5 \\
\text { NR } \\
<0.01 \\
<0.01 \\
<0.01\end{array}$ & $\begin{array}{l}\text { NS } \\
\text { NS } \\
\text { NS } \\
\text { NS } \\
\text { NS }\end{array}$ & $\begin{array}{l}F \\
G\end{array}$ \\
\hline MW5 & $\begin{array}{l}10 / 25 / 01 \\
12 / 12 / 02 \\
12 / 29 / 03 \\
12 / 02 / 05 \\
02 / 20 / 07\end{array}$ & $\begin{array}{l}1.3 \\
\text { NS } \\
1.3 \\
\text { NS } \\
\text { NS }\end{array}$ & $\begin{array}{l}<0.5 \\
\text { NS } \\
<0.5 \\
\text { NS } \\
\text { NS }\end{array}$ & $\begin{array}{l}<0.5 \\
\text { NS } \\
<0.5 \\
\text { NS } \\
\text { NS }\end{array}$ & $\begin{array}{l}<0.5 \\
\text { NS } \\
<0.01 \\
\text { NS } \\
\text { NS }\end{array}$ & $\begin{array}{l}\text { NS } \\
\text { NS } \\
\text { NS } \\
\text { NS } \\
\text { NS }\end{array}$ & \\
\hline MW6 & $\begin{array}{l}10 / 25 / 01 \\
12 / 12 / 02 \\
12 / 29 / 03 \\
12 / 02 / 05 \\
02 / 20 / 07\end{array}$ & $\begin{array}{l}4.3 \\
4.0 \\
3.7 \\
2.1 \\
4.7\end{array}$ & $\begin{array}{l}\quad 0.63 \\
\text { NR } \\
<0.5 \\
<0.5 \\
0.62\end{array}$ & $\begin{array}{l}<0.5 \\
\text { NR } \\
<0.5 \\
<0.5 \\
<0.5\end{array}$ & $\begin{array}{l}<0.5 \\
\text { NR } \\
<0.01 \\
<0.01 \\
<0.01\end{array}$ & $\begin{array}{l}\text { NS } \\
\text { NS } \\
\text { NS } \\
\text { NS } \\
\text { NS }\end{array}$ & $\mathrm{H}$ \\
\hline MW7 & $\begin{array}{l}10 / 25 / 01 \\
12 / 12 / 02 \\
12 / 29 / 03 \\
12 / 02 / 05 \\
02 / 20 / 07\end{array}$ & $\begin{array}{l}8.1 \\
8.2 \\
8.0 \\
4.0 \\
6.2\end{array}$ & $\begin{array}{c}1.7 \\
\text { NR } \\
1.4 \\
0.92 \\
1.0\end{array}$ & $\begin{aligned} & 0.58 \\
N R & \\
< & 0.5 \\
< & 0.5 \\
< & 0.5\end{aligned}$ & $\begin{array}{c}<0.5 \\
\text { NR } \\
\quad 0.022 \\
0.022 \\
<0.01\end{array}$ & $\begin{array}{l}\text { NS } \\
\text { NS } \\
\text { NS } \\
\text { NS } \\
\text { NS }\end{array}$ & I \\
\hline MW8 & $\begin{array}{l}10 / 25 / 01 \\
12 / 12 / 02 \\
12 / 29 / 03 \\
12 / 02 / 05 \\
02 / 20 / 07\end{array}$ & $\begin{array}{l}7.0 \\
8.3 \\
6.9 \\
4.4 \\
6.3\end{array}$ & $\begin{array}{c}1.7 \\
\text { NR } \\
1.5 \\
1.4 \\
1.5\end{array}$ & $\begin{array}{l}<0.5 \\
\text { NR } \\
<0.5 \\
<0.5 \\
<0.5\end{array}$ & $\begin{array}{l}<0.5 \\
\text { NR } \\
<0.01 \\
<0.01 \\
<0.01\end{array}$ & $\begin{array}{l}\text { NS } \\
\text { NS } \\
\text { NS } \\
\text { NS } \\
\text { NS }\end{array}$ & $\begin{array}{l}\mathrm{J} \\
\mathrm{K}\end{array}$ \\
\hline MW9 & $\begin{array}{l}10 / 25 / 01 \\
12 / 12 / 02 \\
12 / 29 / 03 \\
12 / 02 / 05 \\
02 / 20 / 07\end{array}$ & $\begin{array}{l}<1.2 \\
\text { NS } \\
\text { NS } \\
\text { NS } \\
\text { NS }\end{array}$ & $\begin{array}{l}<0.5 \\
\text { NS } \\
\text { NS } \\
\text { NS } \\
\text { NS }\end{array}$ & $\begin{array}{l}<0.5 \\
\text { NS } \\
\text { NS } \\
\text { NS } \\
\text { NS }\end{array}$ & $\begin{array}{l}<0.5 \\
\text { NS } \\
\text { NS } \\
\text { NS } \\
\text { NS }\end{array}$ & $\begin{array}{l}\text { NS } \\
\text { NS } \\
\text { NS } \\
\text { NS } \\
\text { NS }\end{array}$ & \\
\hline
\end{tabular}




\section{TABLE 3.4 (Cont.)}

a Results are from off-site laboratories. Sources of data: Dallen 1998, 1999; KDHE 1997, 1998, 1999a, 2001, 2004, 2006, 2007a.

b Codes for other volatile organic compounds (concentrations in $\mu \mathrm{g} / \mathrm{L}$ ):

A: $12 / 29 / 03$, Bernhardt: chloromethane $=0.72$.

B: $02 / 20 / 07$, Bernhardt: chloromethane $=0.71$.

C: $04 / 21 / 98$, Sandwell: ethylbenzene $=1.0 ;$ xylene $=3.9 ; 1,3,5$-trimethylbenzene $=0.5$;

1,2,4-trimethylbenzene $=0.7$.

D: $12 / 29 / 03$, MW1: chloromethane $=12 ;$ sec-butylbenzene $=8.3 ;$ methyl tert-butyl ether $=6.4$.

E: $12 / 29 / 03$, MW2: chloromethane $=5.8 ; 1,1$-dichloroethane $=0.75$.

$\mathrm{F}: \quad 12 / 29 / 03$, MW4: benzene $=1.7 ;$ tetrachloroethylene $=0.57 ;$ chloromethane $=4.3$.

G: $12 / 02 / 05$, MW4: benzene $=0.51$.

$\mathrm{H}: \quad 12 / 29 / 03$, MW6: chloromethane $=1.6$.

I: $12 / 29 / 03$, MW7: chloromethane $=14$.

$\mathrm{J}: \quad 12 / 29 / 03$, MW8: chloromethane $=7.5$.

K: 12/02/05, MW8: 1,1-dichloroethane $=0.52$.

c NA, not analyzed for this constituent.

d NS, monitoring location not sampled for this constituent in this event.

e NR, result not reported in the investigation reports on file. 


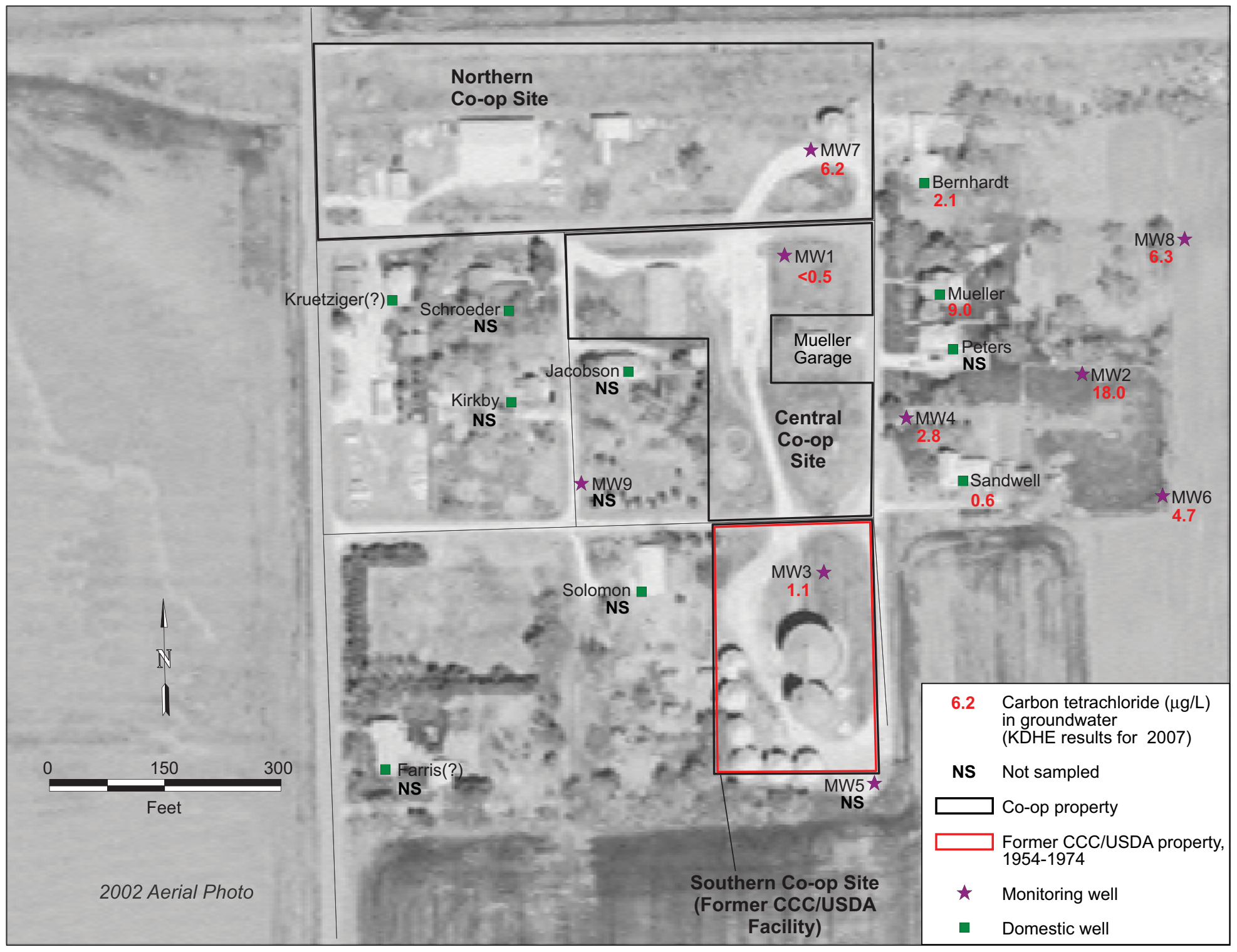

FIGURE 3.1 Analytical results for carbon tetrachloride in groundwater samples collected in 2007. Well locations are approximate. Source of data: KDHE (2007a). Source of photograph: USDA (2002). 


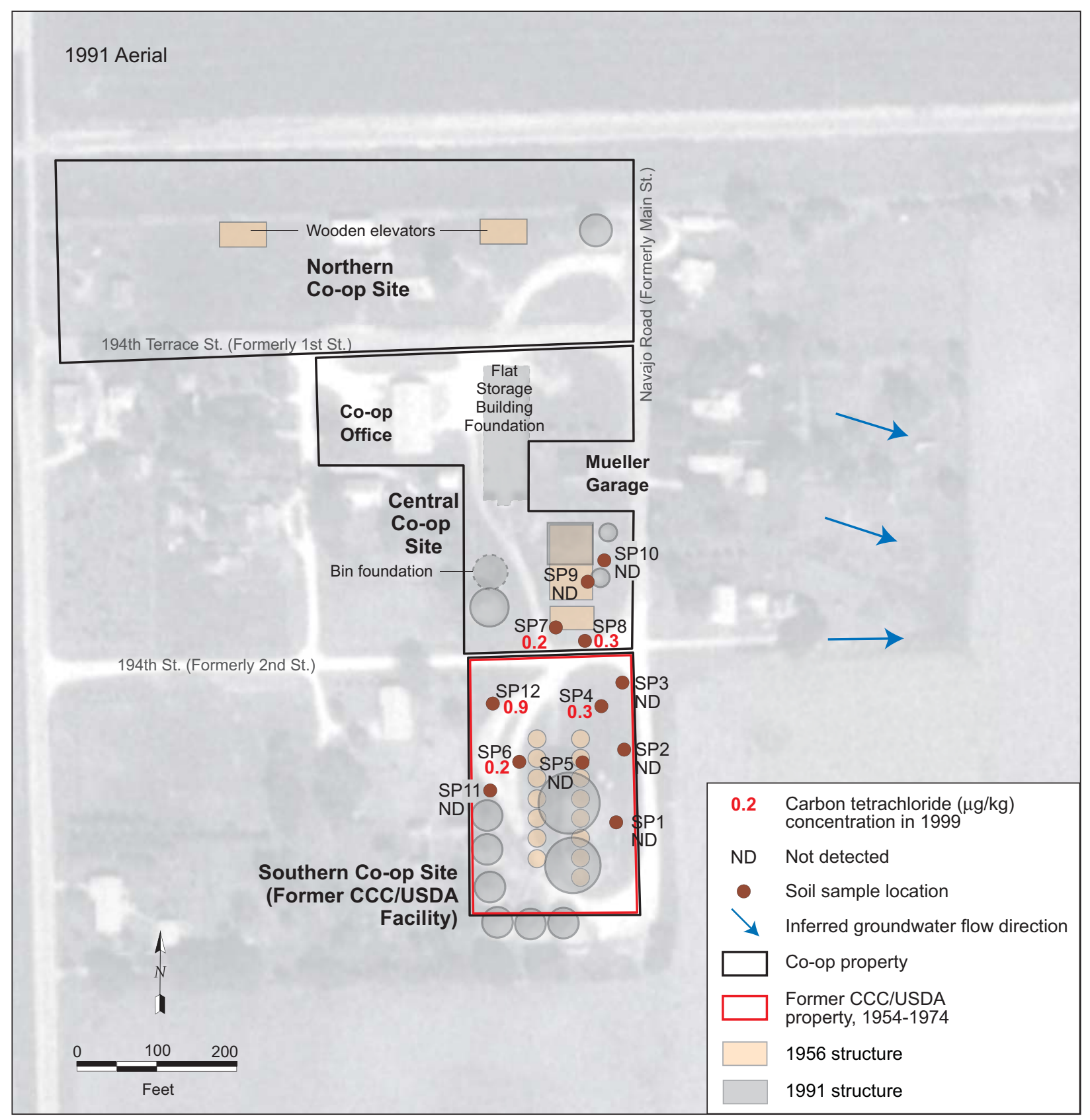

FIGURE 3.2 Analytical results for soil samples collected in November 1999. Source of data: KDHE (1999c). Source of photograph: USGS (1991). 


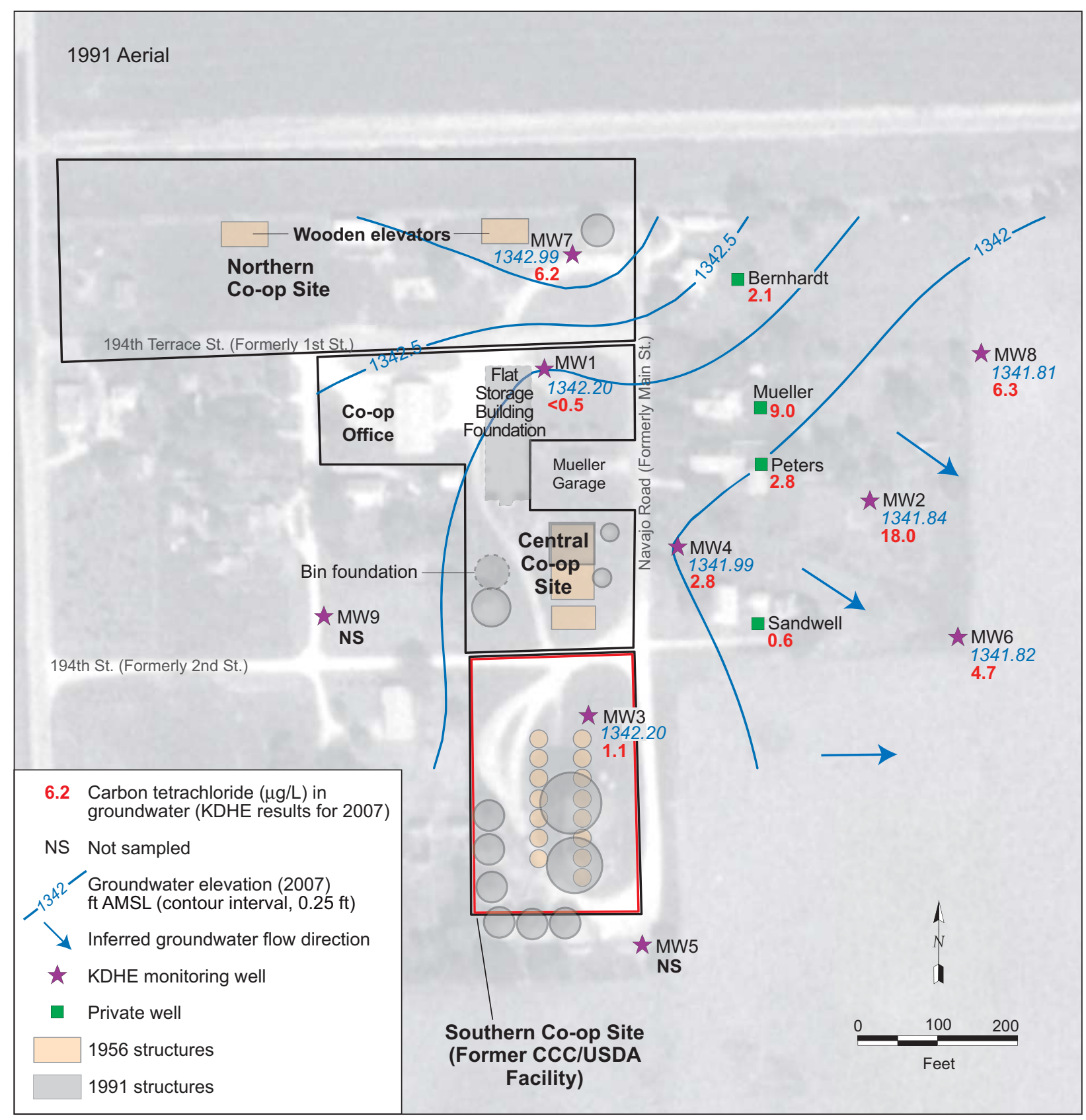

FIGURE 3.3 Structures at the Canada site, 2007 groundwater elevations with interpreted flow direction, and approximate locations of KDHE monitoring wells and private wells with 2007 monitoring results. Source of data: KDHE (2007a). Source of photograph: USGS (1991). 


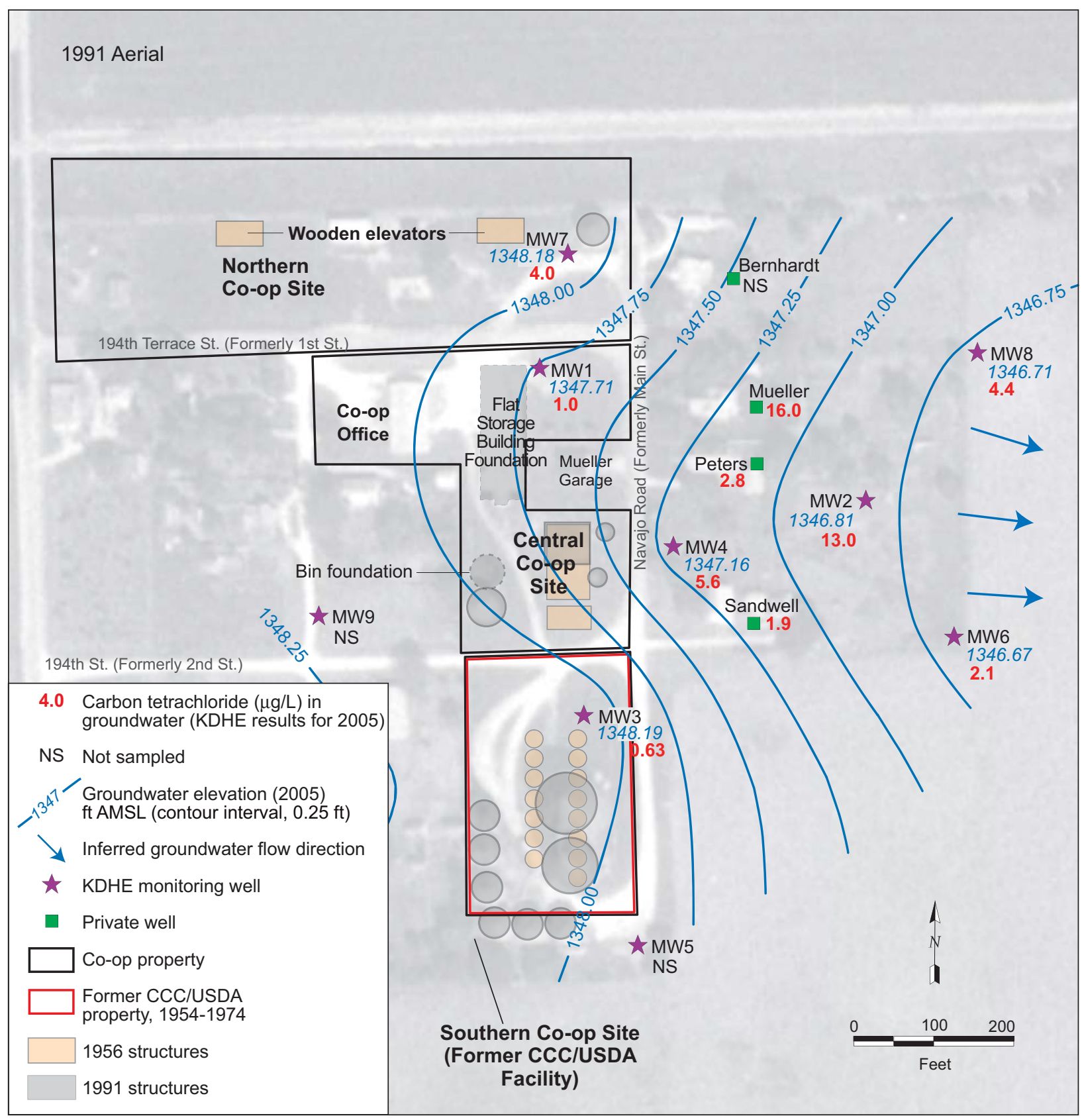

FIGURE 3.4 Structures at the Canada site, 2005 groundwater elevations with interpreted flow direction, and approximate locations of KDHE monitoring wells and private wells with 2005 monitoring results. Source of data: KDHE (2006). Source of photograph: USGS (1991). 


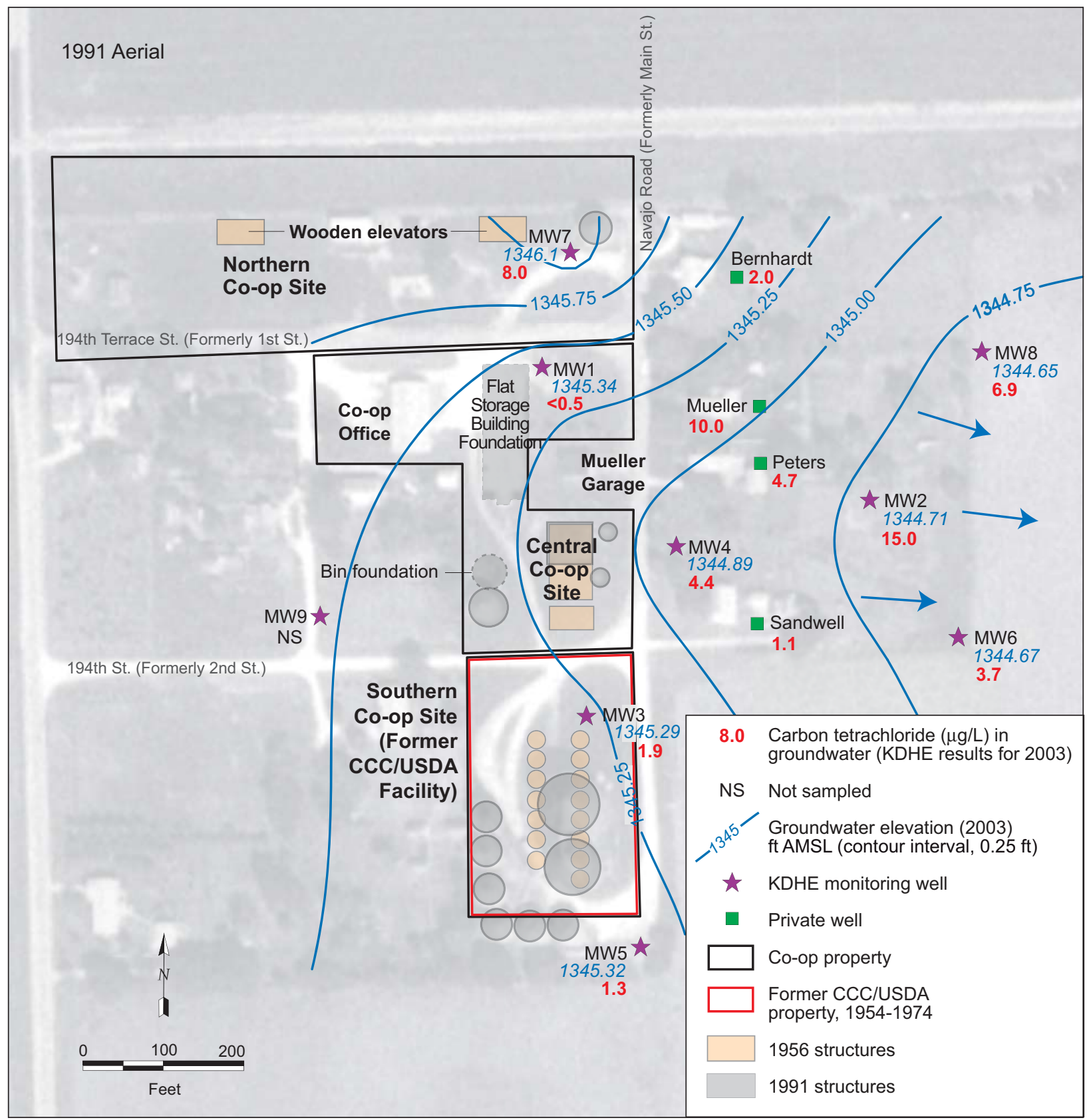

FIGURE 3.5 Structures at the Canada site, 2003 groundwater elevations with interpreted flow direction, and approximate locations of KDHE monitoring wells and private wells with 2003 monitoring results. Source of data: KDHE (2004). Source of photograph: USGS (1991). 


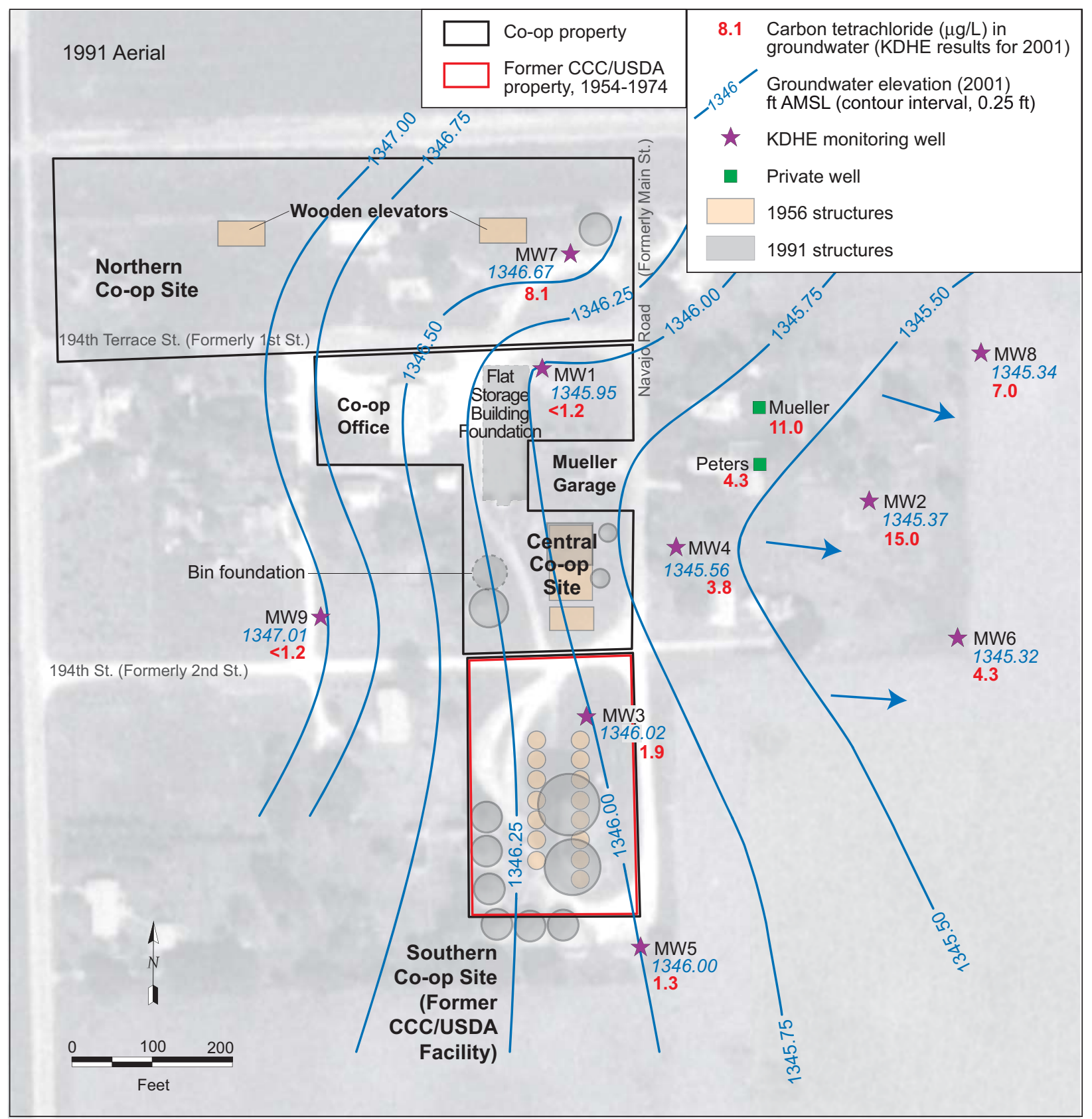

FIGURE 3.6 Structures at the Canada site, 2001 groundwater elevations with interpreted flow direction, and approximate locations of KDHE monitoring wells and private wells with 2001 monitoring results. Source of data: KDHE (2001). Source of photograph: USGS (1991). 


\section{Conclusions}

This evaluation of the KDHE's long-term monitoring data for the period 2001-2007, along with prior investigational data, indicates that the former CCC/USDA facility is an unlikely source for the carbon tetrachloride contamination persistently detected at levels above the MCL at the Canada site. This conclusion is based on the following findings:

- The groundwater flow direction has been persistently measured by the KDHE (2001-2007) as being to the east or east-southeast. This flow direction places the wells with the overall highest concentrations of carbon tetrachloride (e.g., the Mueller and Peters private wells and monitoring wells MW2, MW7, and MW8) downgradient from the northern and central co-op sites, but not downgradient from the former CCC/USDA property. (See Figures 3.3-3.6.)

- During the KDHE's six-year monitoring period (2001-2007), the contaminant level in MW3 (on the former CCC/USDA property) remained well below the MCL of $5.0 \mu \mathrm{g} / \mathrm{L}$, with a concentration of $1.1 \mu \mathrm{g} / \mathrm{L}$ measured in the most recent sampling in February 2007.

- In KDHE soil sampling in 1999, carbon tetrachloride was detected in only one of five soil samples collected between and next to the rows of former CCC/USDA grain bins, at the very low level of $0.2 \mu \mathrm{g} / \mathrm{kg}$ (the method detection limit). Contamination around bin doorways and foundations would most likely be due to CCC/USDA activities. The absence of such a contamination pattern suggests a different source. Carbon tetrachloride was identified with greater frequency (in four of seven samples) and at higher levels $(0.2,0.3,0.3$, and $0.9 \mu \mathrm{g} / \mathrm{kg})$ in soil samples collected north of the former CCC/USDA grain bin array. The co-op has occupied the property to the north of the former CCC/USDA property for an extended period and has occupied the former CCC/USDA property since at least 1974.

- The relatively stable levels of carbon tetrachloride in monitoring wells MW2, MW4, and MW8, downgradient from the flat storage building formerly on the co-op property, suggest the presence of a soil source in the vicinity of that building. 
- The fumigant compounds 1,2-dichloroethane, ethylene dibromide, and tetrachloroethylene were detected in wells downgradient from the central co-op grain storage structures (specifically the now-demolished flat storage building), but not on or downgradient from the former CCC/USDA property. The distribution of these compounds is consistent with a contaminant source other than the former CCC/USDA facility.

- The unique detection of the fumigant compound ethylene dibromide, found only in well MW7 in the KDHE's 2003 and 2005 sampling (Table 3.4), is a potentially significant finding. Well MW7 is downgradient from the former wooden elevators on the northern co-op site, but it is not downgradient from the co-op's former flat storage building or from the former CCC/USDA property (Figures 3.3-3.6). The relatively stable levels of carbon tetrachloride at MW7 (Table 3.4) suggest the presence of a soil source in the vicinity of the former wooden elevators. The presence of ethylene dibromide at only this location further suggests that a different fumigant mixture was used at the former wooden elevators than was used subsequently at the former flat storage building.

- Fuel-related compounds identified at trace levels in the Sandwell private well in 1998 and in monitoring wells MW1, MW4, and MW7 in 2003 and 2005 could be related to a documented release from an underground storage tank at the co-op in 1991 (KDHE 2004, 2007b). Such a connection would demonstrate the viability of a contaminant migration pathway from the co-op to wells to the east.

- KDHE policy BER-RS-024, Rev. 2001, Section I (Groundwater and Surface Water Criteria), indicates the following: "Status reclassification may be granted after monitoring demonstrates cleanup goals have been achieved and maintained for four (4) consecutive, equally time-sequenced sampling episodes conducted under KDHE oversight over a period of no less than two (2) years. ..." The KDHE policy, Section III (Other Considerations), further provides the following: "If the above six (6) criteria do not apply to a site, the PRP may elect to submit to KDHE/BER a Reclassification Petition, including a Reclassification Report as outlined below, that identifies and justifies a 
rationale for reclassifying the site to Resolved status on the ISL despite failure to satisfy the criteria identified above. KDHE will make the final determination concerning reclassification of the site on the ISL and will document that decision accordingly."

The KDHE has conducted seven sampling events at the Canada site over a period of six years, at approximately annual intervals (except for the two-year gap between the December 2003 and December 2005 events). In this KDHE sampling, monitoring well MW3, on the former CCC/USDA property, has exhibited the following carbon tetrachloride concentrations:

$1.6 \mu \mathrm{g} / \mathrm{L}$ (January 5, 2001)

$1.4 \mu \mathrm{g} / \mathrm{L}$ (March 20, 2001)

$1.9 \mu \mathrm{g} / \mathrm{L}($ October 25, 2001)

$2.9 \mu \mathrm{g} / \mathrm{L}$ (December 12, 2002)

$1.9 \mu \mathrm{g} / \mathrm{L}$ (December 29, 2003)

$0.63 \mu \mathrm{g} / \mathrm{L}$ (December 2, 2005)

$1.1 \mu \mathrm{g} / \mathrm{L}$ (February 20, 2007)

These results, which are all well below the MCL of $5.0 \mu \mathrm{g} / \mathrm{L}$, demonstrate that groundwater on the former CCC/USDA property at Canada has met the standard for carbon tetrachloride in drinking water since 2001 and does not pose a significant human health threat. The results warrant a request for reclassification of the Canada site to Resolved status on the KDHE's Identified Sites List, under Section III (Other Considerations) of KDHE policy BER-RS-024, Rev. 2001. 


\section{References}

CGS, 2007, Canada, Cooperative Grain and Supply, Hillsboro, Kansas, http://www.cgsmc.com/ locations/canada.html, January 26.

Byrne, F.E., C.P. Walters, J.L. Hill, and L. Riseman, 1959, "Geology and Construction-Material Resources of Marion County, Kansas," Chapter B (pp. 63-95) in Geology and Construction Materials of Part of Northeast Kansas, U.S. Geological Survey Bulletin 1060, U.S. Government Printing Office, Washington, D.C.

Chaffee. P., 1988, Effects of Water-Well Construction on Temporal Variability of Ground-Water Quality in Lincolnville, Marion County, Kansas, Kansas Geological Survey Open-File Report 88-26.

Dallen, F., 1998, memorandum from Dallen (Environmental Technician) to R. Brown (PreRemedial Unit Chief, Pre-Remedial Unit/Remedial Section, Bureau of Environmental Remediation, Kansas Department of Health and Environment, Topeka, Kansas), regarding a trip report for private well sampling conducted on December 3, 1997, for the KDHE's Pre-CERCLIS site reconnaissance and evaluation of the former CCC/USDA grain bin site in Canada, Kansas, February 12 .

Dallen, F., 1999, memorandum from Dallen (Environmental Technician) to R. Brown (PreRemedial Unit Chief, Pre-Remedial Unit/Remedial Section, Bureau of Environmental Remediation, Kansas Department of Health and Environment, Topeka, Kansas), regarding a trip report for subsurface soil and private well sampling conducted on February 23-24, 1999, for the KDHE's preliminary assessment/screening site inspection of the former CCC/USDA grain bin site in Canada, Kansas, March 11.

FSA, 1997, Former CCC Grain Bin Storage Facilities Survey - January 1997, completed for the Canada Site by B. Harmon (Marion, Kansas), Farm Services Administration, U.S. Department of Agriculture, January 29.

Jordan, P.R., and R.J Hart, 1985, Transit Losses and Travel Times for Water-Supply Releases from Marion Lake during Drought Conditions, Cottonwood River, East-Central Kansas, Water Resources Investigations Report 85-4263, U.S. Geological Survey. 
KDHE, 1997, record of sampling conducted on October 21, 1997, by S. Lang and H. Debauche, for the CCC/USDA private well sampling program, Bureau of Environmental Remediation, Kansas Department of Health and Environment, Topeka, Kansas.

KDHE, 1998, Pre-CERCLIS Site Reconnaissance and Evaluation: Canada Former USDA/CCC Grain Bin Site, Canada, Marion County, Kansas, KDHE ID P505770601, Bureau of Environmental Remediation, Kansas Department of Health and Environment, Topeka, Kansas, March.

KDHE, 1999a, Preliminary Assessment/Screening Site Inspection: Canada Former USDA/CCC Grain Bin Site, Canada, Marion County, Kansas. CERCLIS Identification Number KSSFN0703499, Bureau of Environmental Remediation, Kansas Department of Health and Environment, Topeka, Kansas, April.

KDHE, 1999b, Preliminary Removal Site Evaluation/Removal Preliminary Assessment Form: Canada Former USDA/CCC Grain Bin Site, Canada, Marion County, Kansas. CERCLIS Identification Number KSSFN0703499, completed by R.L. Brown (Unit Chief, Site Assessment Unit, Bureau of Environmental Remediation, Kansas Department of Health and Environment, Topeka, Kansas), April 19; reviewed by R.L. Bean (Section Chief, Remedial Section, Bureau of Environmental Remediation, Kansas Department of Health and Environment, Topeka, Kansas), June 22.

KDHE, 1999c, Supplemental Sampling Assessment: Canada Former USDA/CCC Grain Bin Site, Canada, Marion County, Kansas, KDHE ID P5-057-70601, completed by F. Dallen (Site Assessment Unit, Bureau of Environmental Remediation, Kansas Department of Health and Environment, Topeka, Kansas), November.

KDHE, 2001, Final Report: Comprehensive Investigation: Canada USDA/CCC Grain Bin Site, Canada, Kansas, prepared by Geotechnical Services, Inc., Wichita, Kansas, for Kansas Department of Health and Environment, Topeka, Kansas, December.

KDHE, 2004, 2003 State Water Plan Long Term Monitoring Results: Canada Carbon Tetrachloride Site, Canada, Marion County, memorandum from B. Conrad to B. Wilson, Kansas Department of Health and Environment, Topeka, Kansas, March 5. 
KDHE, 2006, 2005 State Water Plan Long Term Monitoring Results: Canada Carbon Tetrachloride Site, Canada, Marion County, memorandum from B. Wilson to Canada Site File, Kansas Department of Health and Environment, Topeka, Kansas, January 5.

KDHE, 2007a, Memorandum from J. Vopta to D. Doubek for Canada Carbon Tetrachloride Site File, Bureau of Environmental Remediation, Kansas Department of Health and Environment, Topeka, Kansas, March 9.

KDHE, 2007b, Assessed Storage Tank Facilities, Storage Tank Section, Kansas Department of Health and Environment, Topeka, Kansas, updated March 5, 2007, http://www.kdheks. gov/tanks/download/astf_db.xls, accessed August 9, 2007.

O'Connor, H.G., and P.K. Chaffee, 1992, A Time-Series Water Quality Study, Lincolnville, Pilsen, and Ramona, Marion County, Kansas, Open-File Report 92-56, Kansas Geological Survey.

Shewey, C., 1999, record of telephone conversation involving Shewey (Marion County Rural Water District, Hillsboro, Kansas), regarding Rural Water District connection to Canada, Kansas, residences, July 21.

State of Kansas, 1883, plat survey of Canada, Kansas, recorded with the State of Kansas, Marion County, 4:00 p.m., December 15.

Terman, M.R., and J.S. Aber, 1994, Fall Field Trip in Marion County, Central Kansas, Kansas Academy of Science Multidisciplinary Guidebook 7, Kansas Geological Survey Open-File Report 94-33.

USACE 1974, Final Environmental Statement, Marion, Kansas, Local Protection Project, U.S. Army Corps of Engineers, Tulsa District.

USDA, 1956, aerial photograph AYG-2R-203D-1W, U.S. Department of Agriculture, Washington, D.C., June 12.

USDA, 2002, Orthophoto Mosaic for Marion County, Kansas, Natural Resources Conservation Service, U.S. Department of Agriculture, Washington, D.C. 
USGS, 1985, Canada Quadrangle, Marion County, Kansas, U.S. Geological Survey 7.5-Minute Topographic Map Series, U.S. Government Printing Office, Washington, D.C.

USGS, 1991, Digital Orthophoto Quarter Quadrangle, U.S. Geological Survey, Washington, D.C., obtained from Kansas Data Access and Support Center, Kansas Geological Survey, University of Kansas, Lawrence, Kansas. 


\section{Appendix A:}

Well Registration Forms and Drilling Records for Canada Area Wells 


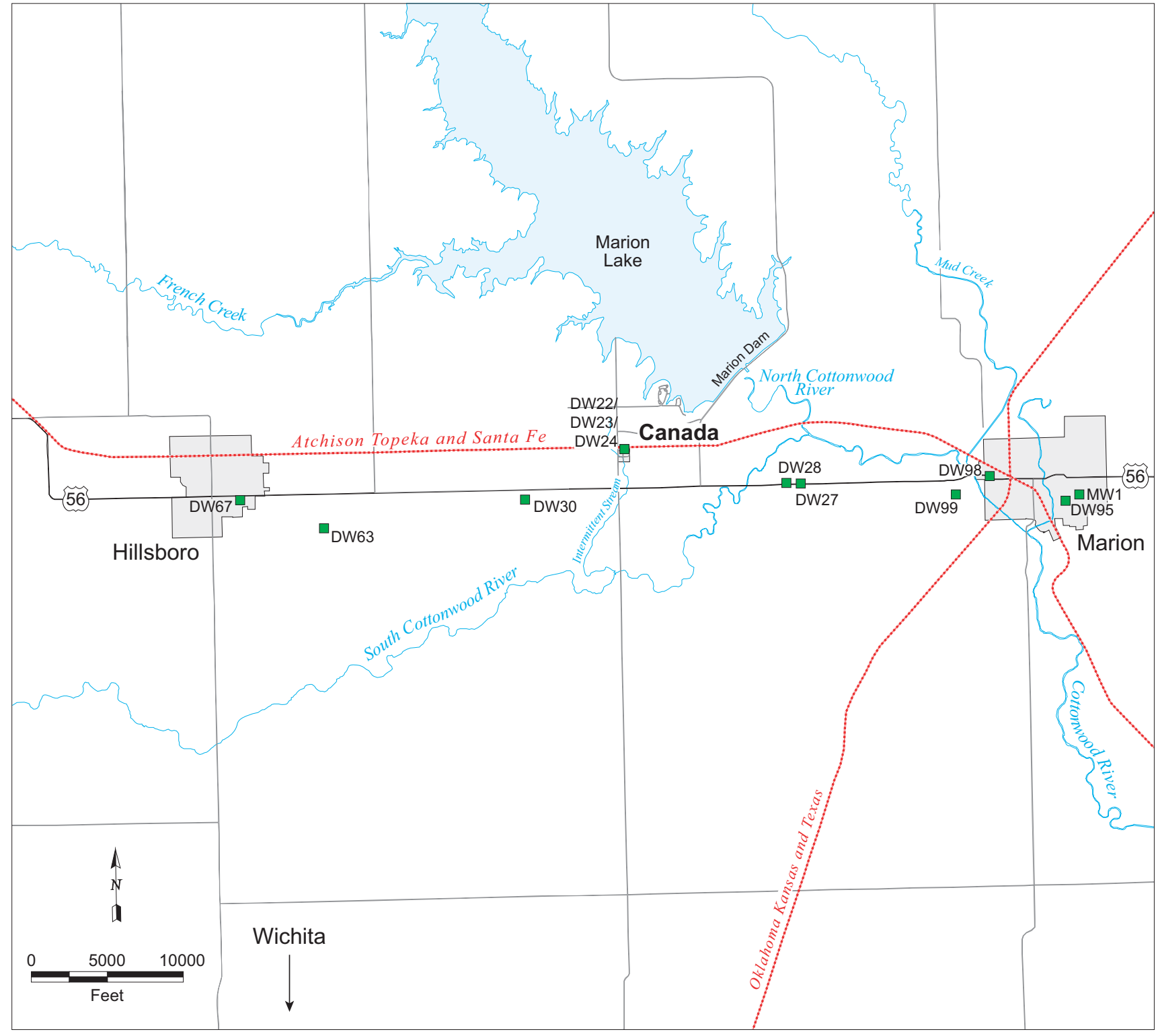

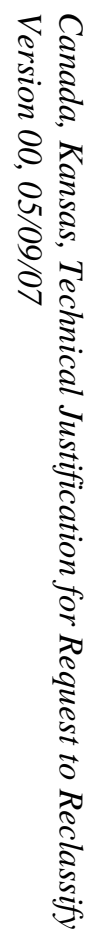

FIGURE A.1 Locations of registered wells in the Canada area. 


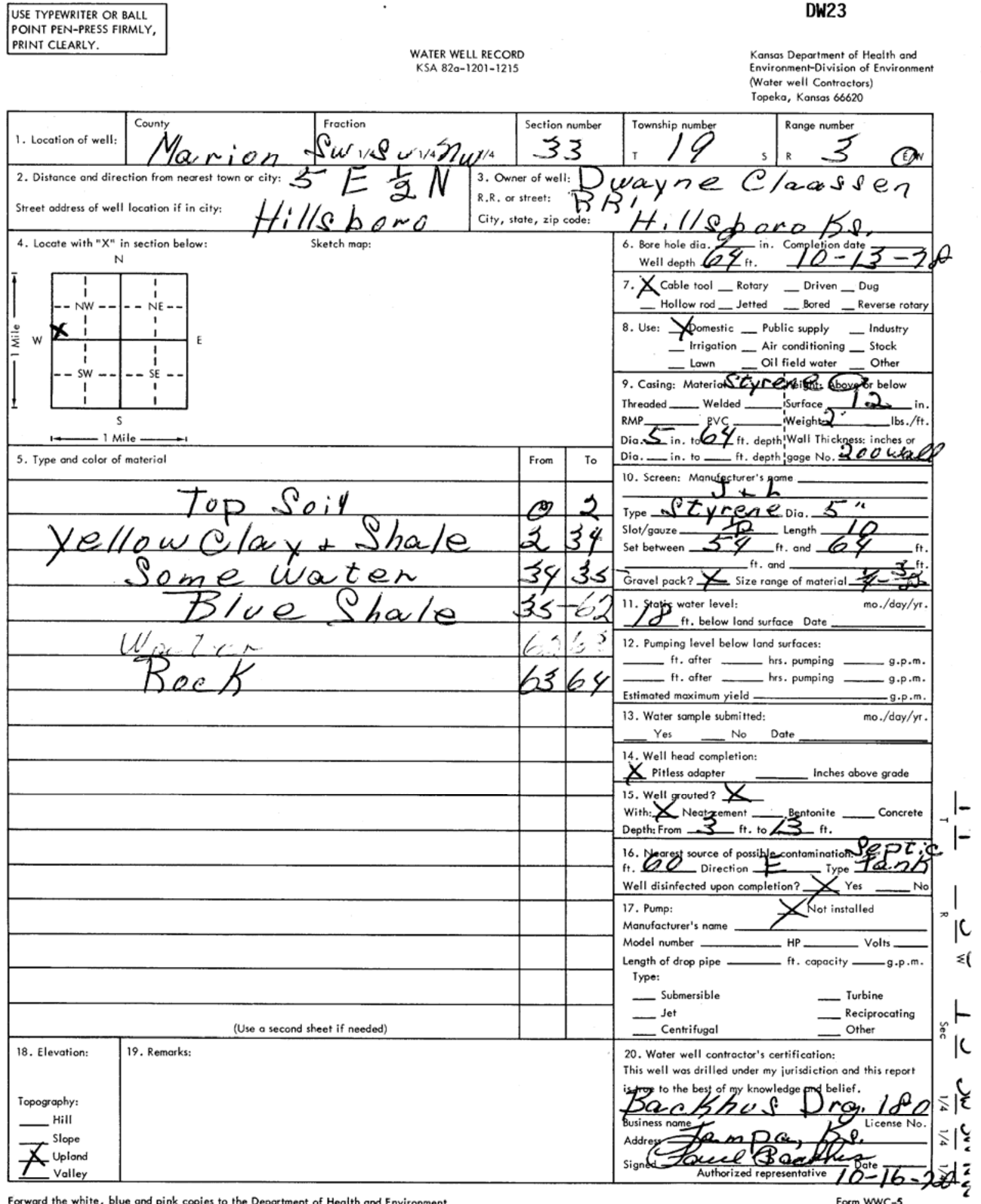


WATER WELL RECORD Form WWC-5 KSA 82a-1212

DW24

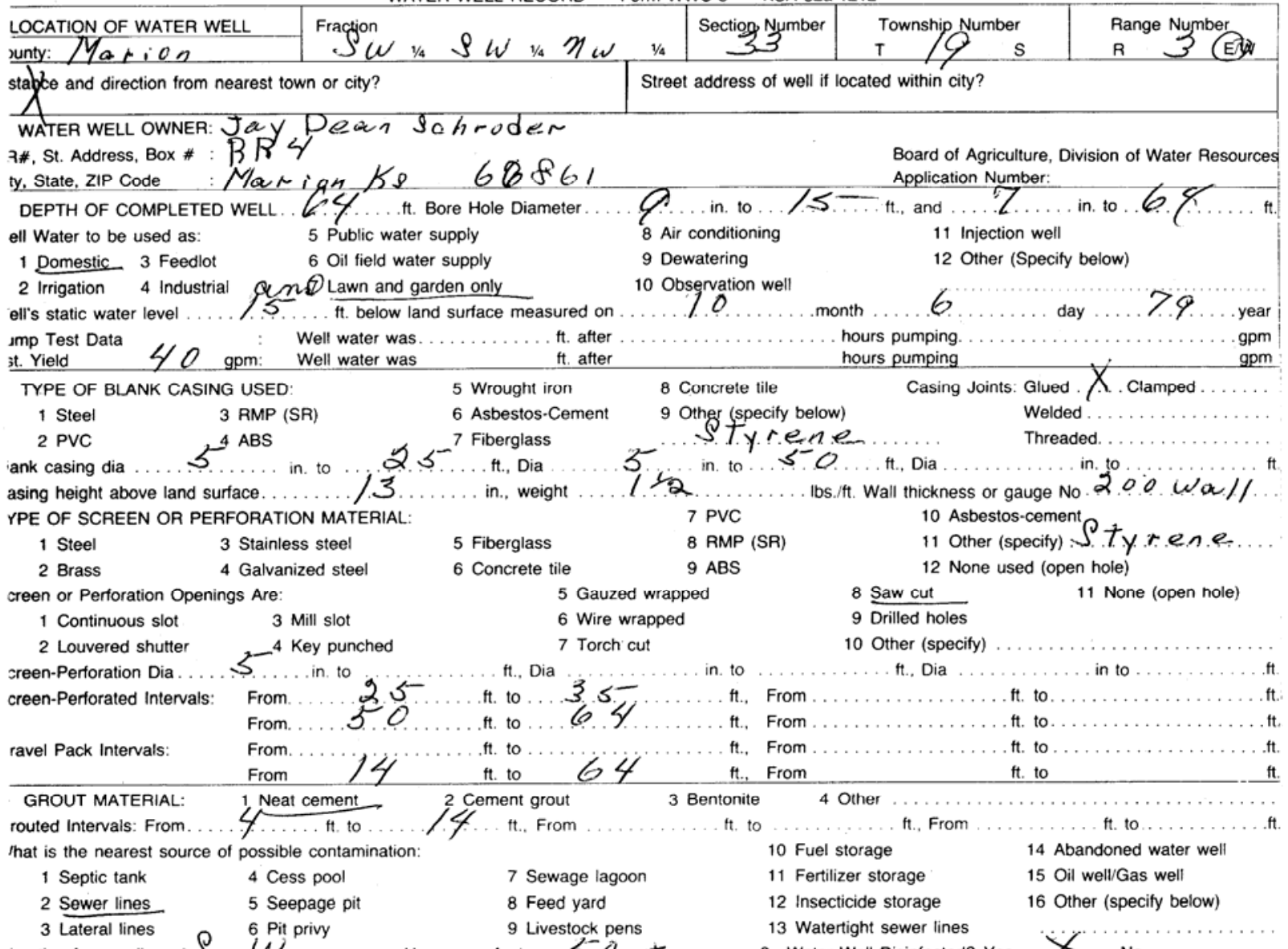

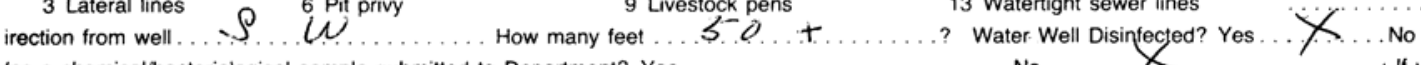

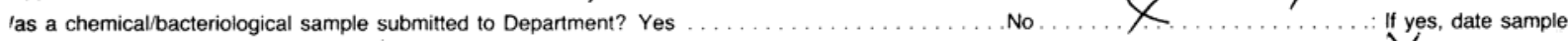

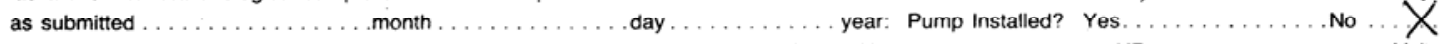

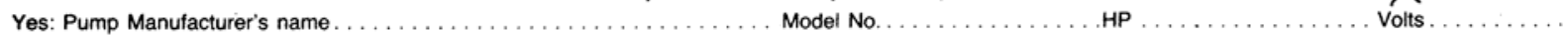

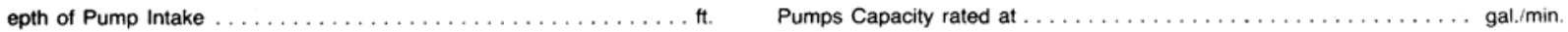

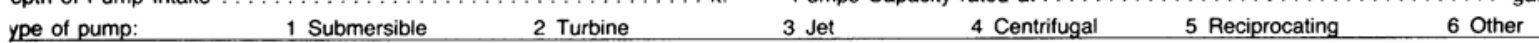

CONTRACTOR'S OR LANDOWNER'S CERTIFICATION: This water well was (1) constructed, (2) reconstructed, or (3) plugged under my jurisdiction and was

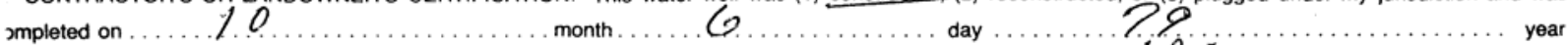

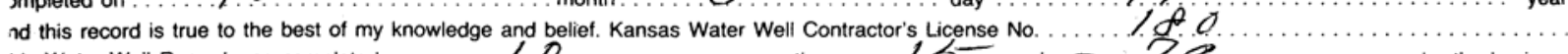

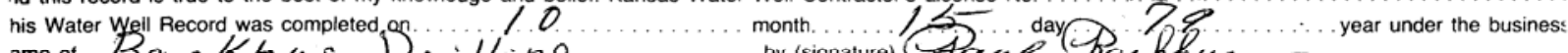
ame of Baelshos Drilling by (signature sace (ibeakhe \begin{tabular}{|l|ll|l|l|l|l|}
\hline LOCATE WELL'S LOCATION & FROM & TO & LITHOLOGIC LOG & FROM & TO & LITHOLOGIC LOG
\end{tabular} WITH AN " $X$ " IN SECTION BOX:

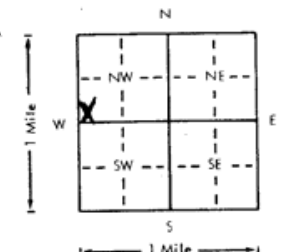

LEVATION:

lepth(s) Groundwater Encountered

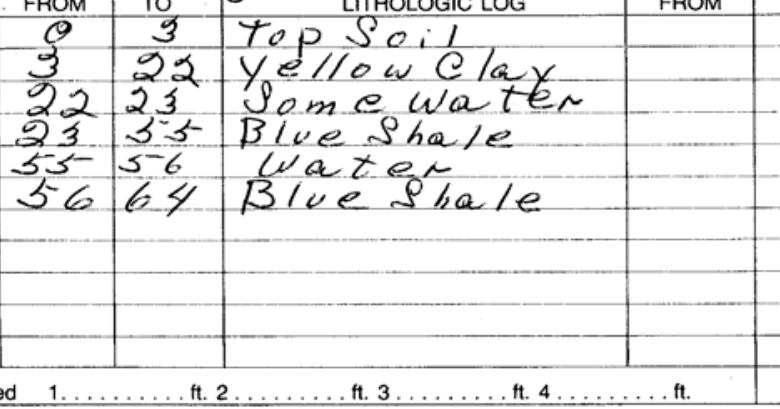

\begin{tabular}{|c|c|}
\hline TO & LITHOLOGIC LOG \\
\hline & \\
\hline & \\
\hline & \\
\hline & \\
\hline & \\
\hline & \\
\hline & \\
\hline
\end{tabular}

NSTRUCTIONS: Use typewriter or ball point pen, please press firmly and PRINT clearly. Please fill in blanks, underline or circle the correct answers. Send top three opies to Kansas Department of Health and Environment, Division of Environment, Water Well Contractors, Topeka, KS 66620. Send one to WATER WELL OWNER and tain one for your records. 
DW27

\begin{tabular}{|c|c|c|c|c|}
\hline $\begin{array}{l}\text { LOCATION OF WATER WELL: } \\
\text { ounty: }\end{array}$ & 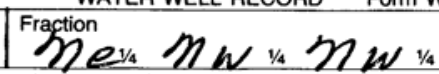 & Sectionglumber & $\begin{array}{lll}\text { Township Number } \\
T \quad 2 & 0 & s\end{array}$ & Range Number \\
\hline
\end{tabular}
istance and direction from nearest town or city street address of well if located within city? $23 y$ marion

WATER WELL OWNER: han en ence Win Kley R*, St. Address, Box \#: RRI : Majion, KS, $66 \mathrm{R}, 1$

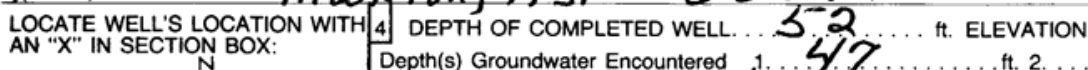

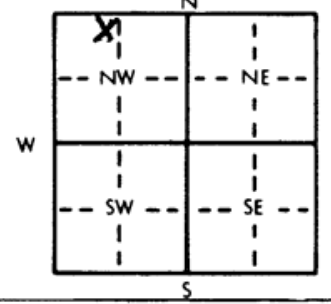

TYPE OF BLANK CASING USED:

1 Steel

2 PVC

3 RMP (SR)

$4 \mathrm{ABS}$

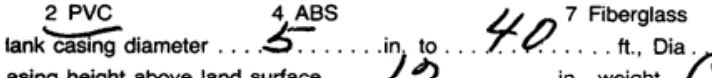

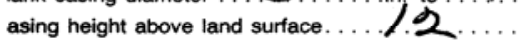

YPE OF SCREEN OR PERFORATION MATERIAL:

1 Steel

3 Stainless steel

2 Brass

4 Galvanized steel

CREEN OR PERFORATION OPENINGS ARE

1 Continuous slot 3 Mill slot

2 Louvered shutter 4 Key punched

CREEN-PERFORATED INTERVALS:

\section{Fiberglass}

6 Concrete tile
Pyinp tes

$\mathrm{gp}$ ( Well water was
Bore Hole Diameter. $2 \frac{1}{2}$ in. to 52

Domestic 3 Feedlot

4 Industrial

5 Public water supply

7 Lawn and garden only

submitted to Department? Yes mitted From

Board of Agriculture, Division of Water Resource Application Number:

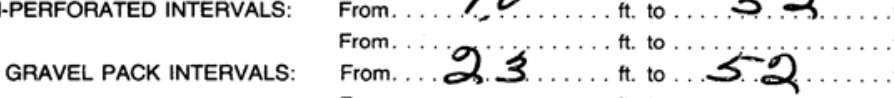

GRAVEL PACK INTERVALS: $\quad$ From....2. $3 \ldots \ldots$ tt. to .... $3 . . .$.
5 Gauzed wrapped

6 Wire wr

7 Torch cut
8 Concrete tile

9 Other (specify below) Water Well Disinfected? Yes No

CASING JOINTS: Glued . . . Clamped . . . . .

Welded.................

Threaded. . . . . . . . . . . .

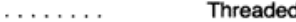

..ft. 3.

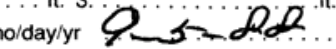

hours pumping ......... gpm

urs pumping $\ldots \ldots \ldots$ gpm

11 Injection well

2 Other (Specity below)

GROUT MATERIAL: 1 Neat cement

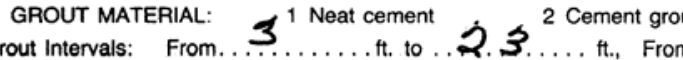

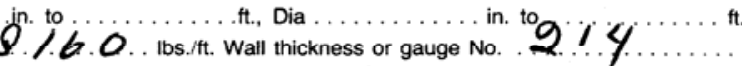

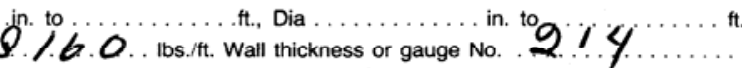

7 PVC 10 Asbestos-cement

8 RMP (SR)

11 Other (specify)

12 None used (open hole)

8 Saw cut

11 None (open hole)

9 Drilled holes

10 Other (specity)

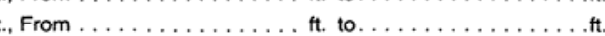

, From $\ldots \ldots \ldots \ldots \ldots \ldots$ ft. to $\ldots \ldots \ldots \ldots \ldots$ ft ft., From

ft. to 109

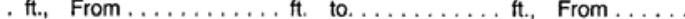

at is the nearest source of possible contamination
1 Septic tank
4 Lateral lines
2 Sewer lines 5 Cess pool
3 Watertight sewer lings 6 Seepage pit
7 Pit privy
Sewage lagoon

10 Livestock pens

14 Abandoned water well

11 Fuel storage

15 Oil well/Gas well

12 Fertilizer storage

16 Other (specify below)

irection from well?

FAOM TO T

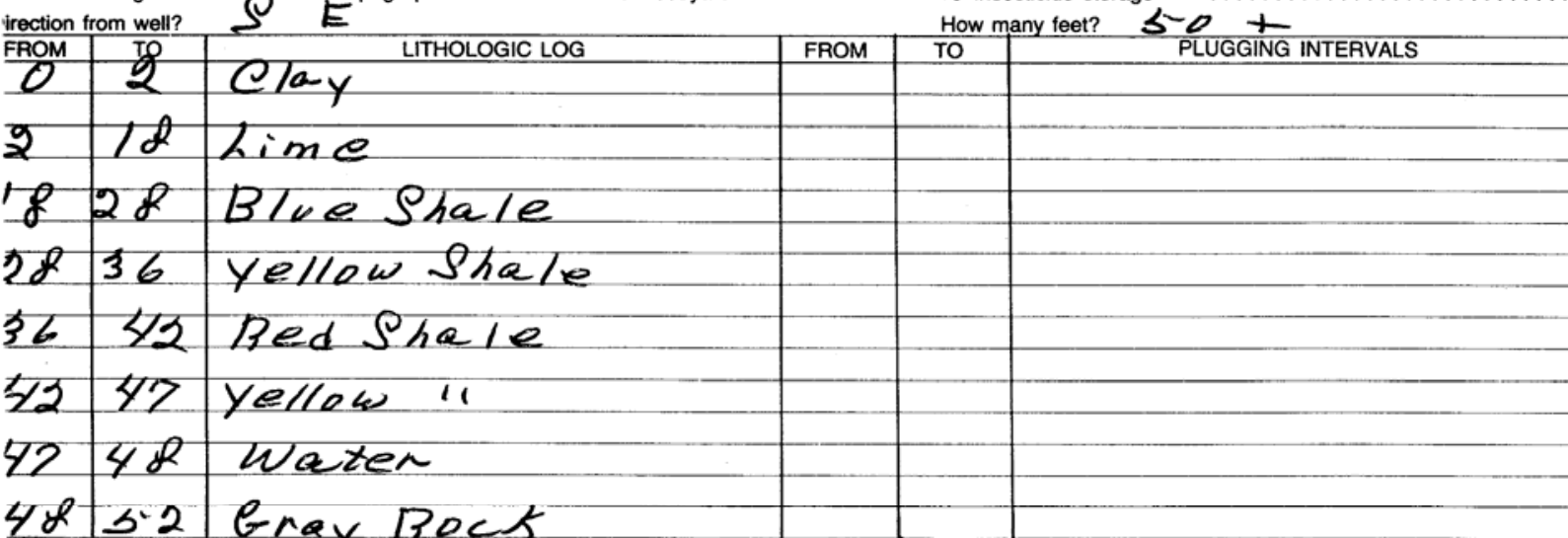

CONTRACTOR'S OR LANDEWNER'S CERTIFICATPN: Jhis water well was (1) constructed, (2) reconstructed, or (3) plugged under my jurisdiction and was

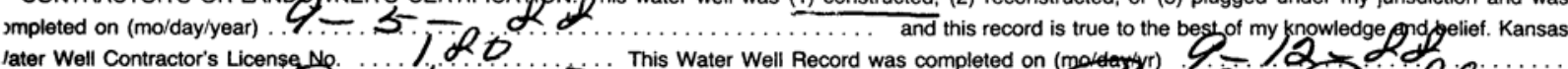
This Water Well Record was completed on (modeayr) 
DW28

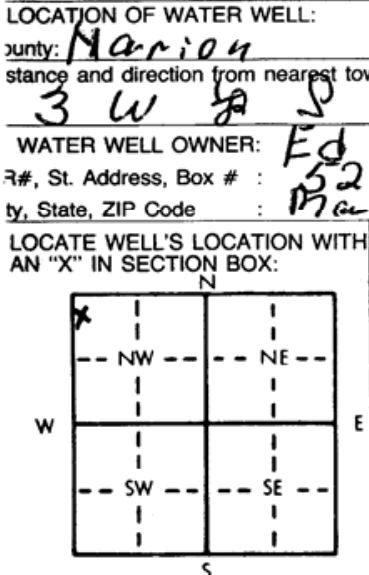

WATER WELL RECORD Form WWC-5 KSA 82a-1212

DW28

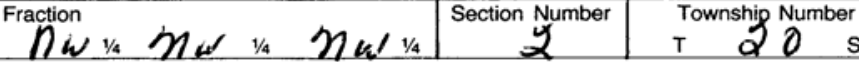
or city street address of well if located within city? Oar a $\sim 0.1$

WATER WELl OWNER: Ed gehrpeder ty, State, zip code: Phar.04 KS. 66061

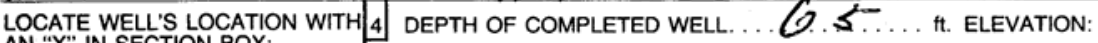
AN "X" IN SECTION BOX: Depth(s) Groundwater Encountered

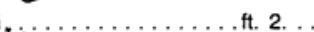

Board of Agriculture, Division of Water Resources Application Number:

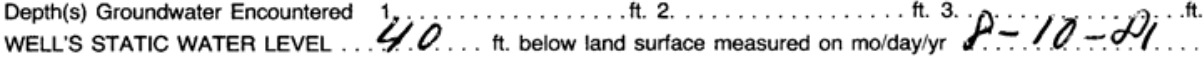
Pypp test data: Well water was $\ldots \ldots \ldots \ldots$ ft. after $\ldots \ldots \ldots$ hours pumping $\ldots \ldots \ldots \ldots$ gpm

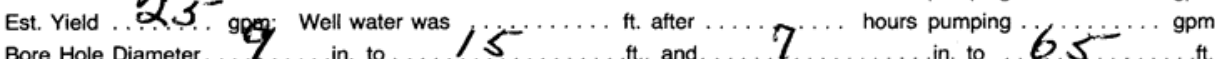
WELL WATER TO BE USED AS: 5 Public water supply 8 Air conditioning 11 Injection well 1 Domestic 3 Feedlot 6 Oil field water supply 9 Dewatering 12 Other (Specify below) 2 Irrigation 4 Industrial 7 Lawn and garden only 10 Observation well Was a chemical/bacteriological sample submitted to Department? Yes...................... if yes, mo/day/yr sample was submitted Water Well Disinfected? Yes $X$ No

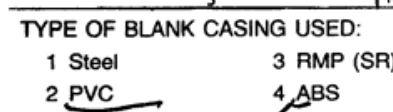

2 PVC 4 ABS

ank casing diameter .... $、 \ldots \ldots$. . . .

asing height above land surface..... YPE OF SCREEN OR PERFORATION MATERIAL:

$$
1 \text { Steel }
$$

3 Stainless steel

2 Brass 4 Galvanized steel CREEN OR PERFORATION OPENINGS ARE

1 Continuous slot

2 Louvered shutter 3 Mill slot 4 Key punched

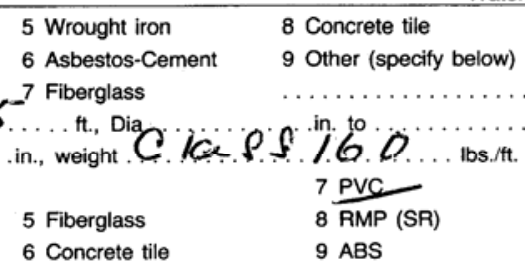

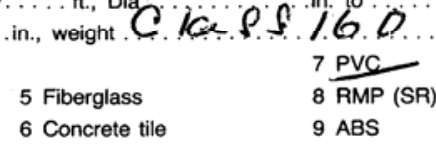
CASING JOINTS: Glued . . . Clamped . . . . .

\section{$4 . \mathrm{K}^{7}$ Fiberglass}

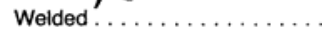
Threaded. . . . . . . . . . . to 60 . 6 Wire wrapped hickness or gauge No.

10 Asbestos-cement

11 Other (specify)

12 None used (open hole) CREEN-PERFORATED INTERVALS: GRAVEL PACK INTERVALS: From. .... 46 ft. to ......

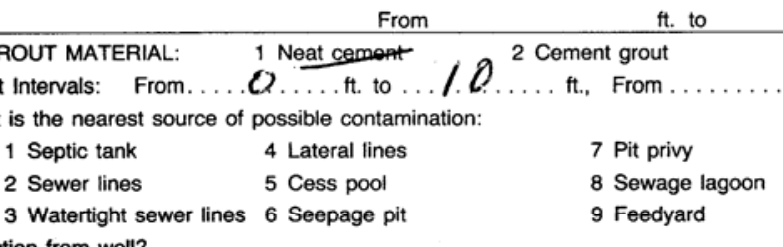

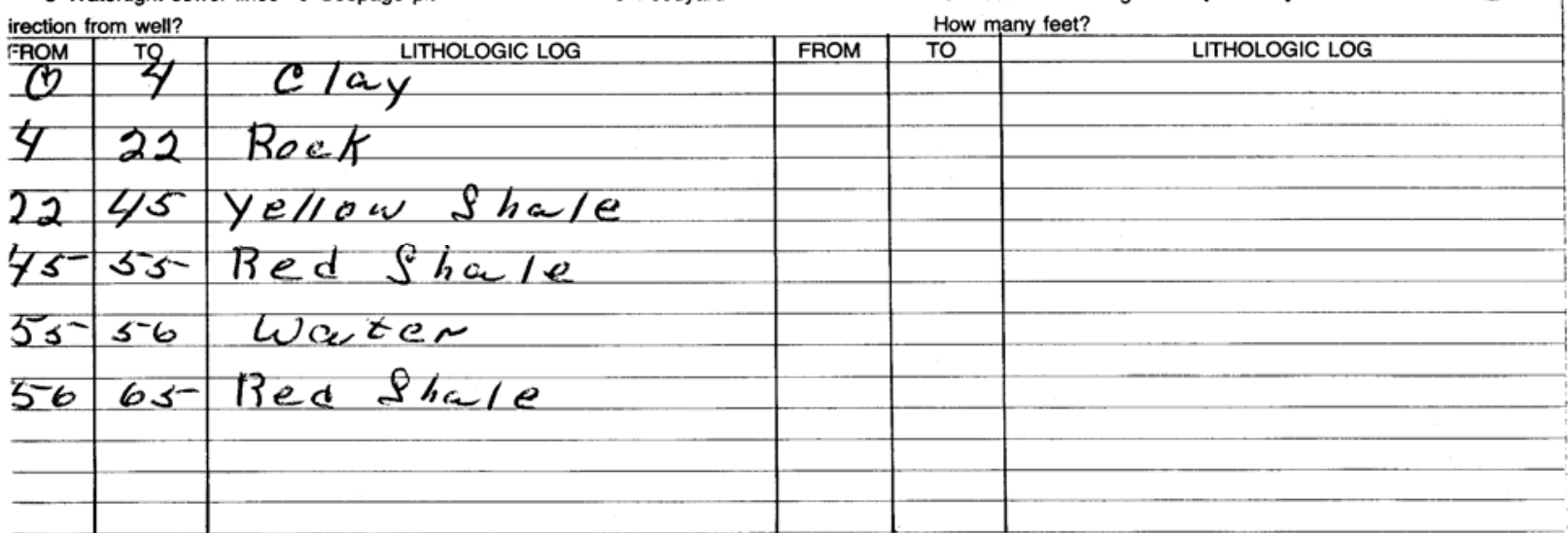

CONTRACTOR'S OR LANDQYVNER'SCERTIFICATION:OThis water well was (1) constructed. (2) reconstructed, or (3) plugged under my jurisdiction and was mpleted on (mo/day/year) .

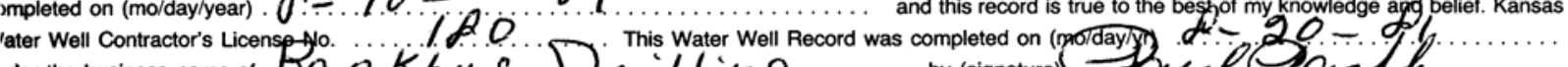
der the business name of $\mathrm{PQ} C \mathrm{CH}$ ISTRUCTIONS: Use typewriter or ball point pen, PLEASE PRESS FIRMLY and 6 RINT clearly. Please fill in blanks, underline or circle the correct answers. Send top ree copies to Kansas Department of Hea
iWNER and retain one for your records. 
DW30

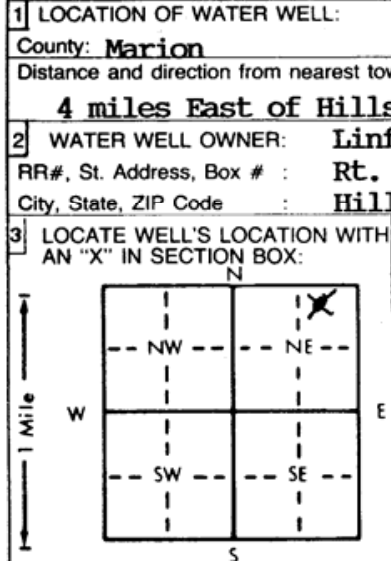

WATER WELL RECORD

Form WWC-5 KSA 82a-1212 Fraction Section Numbe

Township Number
T $20 \quad$ S

\begin{tabular}{c|c}
\hline sumer & \\
\hline
\end{tabular}

Range Number

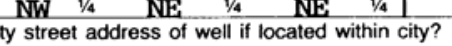
6 oro, KS Linford Funk Rt. 2, Box 100 Hillsboro, KS 67063

Board of Agriculture, Division of Water Resource Application Number: N/A

5 TYPE OF BLANK CASING USED:

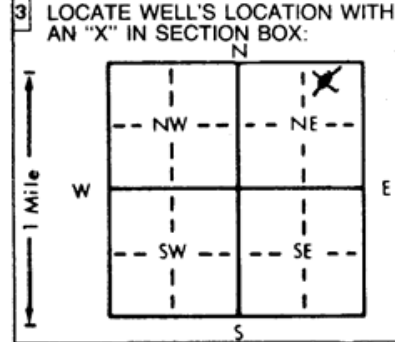

XPVC

4 ABS

Blank casing diameter . . . 5. . . .

Casing height above land surface . . . in. to . . 45

TYPE OF SCREEN OR PERFORATION MATERIAL

1 Steel

3 Stainless stee

2 Brass

4 Galvanized steel

SCREEN OR PERFORATION OPENINGS ARE:

1 Continuous slot

$\mathrm{X}$ Mill slot

2 Louvered shutter

4 Key punched

SCREEN-PERFORATED INTERVALS:

GRAVEL PACK INTERVALS:

From. .

From

6 GROUT MATERIAL: 1 Neat cement

From. . . 0 .....ft. to ... 20 20 Cement grou

What is the nearest source of possible contamination:

$\begin{array}{ll}\text { XSeptic tank } & 4 \text { Lateral lines } \\ 2 \text { Sewer lines } & 5 \text { Cess pool } \\ 3 \text { Watertight sewer lines } & 6 \text { Seepage pit }\end{array}$

3 Watertight sewer lines

DEPTH OF COMPLETED WELL. .

(a)

.....ft., Dia.

6 Concrete tile

ft., From.
$55 \ldots \ldots$ th. Elevation:

tt. below land surface measured on mo ft. 3.

ft. $3 .$.

$3 / 23 / 98$

Pump test data: Well water was $\ldots \ldots \ldots \ldots$ th after $\ldots \ldots \ldots$ hours pumping $\ldots \ldots \ldots$ gpm

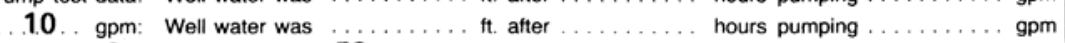

WLTER TO BE USED AS: $\quad 5$ Public water supply $\quad 8$ Air conditioning $\quad 11$ Injection well

XDomestic $\quad 3$ Feedlot 6 Oil field water supply 9 Dewatering 12 Other (Specify below)

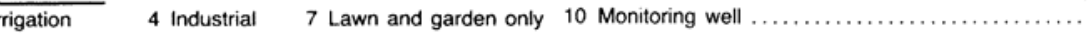

a chemical/bacteriological sample submitted to Department? Yes............No.... X ..... If yes, mo/day/yr sample was sub-

5 Wrought iron
6 Asbestos-Cement
7 Fiberglass

8 Concrete tile

Other (specify below)

CASING JOINTS: Glued . . . . . Clamped . .

Welded..............

Threaded.

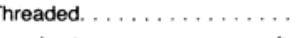

in., weight . . 2.37

in. to

.ft., Dia .

in to 214

........ . Ibs./tt. Wall thickness or gauge No.

\begin{tabular}{lll}
5 Fiberglass & X PVC & 10 Asbestos-cement \\
\hline RMP (SR) & 11 Other (specify).
\end{tabular}

9 ABS

12 None used (open hole)

8 Saw cut

5 Gauzed wrapped

9 Drilled holes

6 Wire wrapped

7 Torch cut

10 Other (specify)

11 None (open hole)

$45 \ldots \ldots$ tt. to .... 55

55 . From

ft. to

ft. to ... 55

ft., From . . . . . . . . . ft. to .

in to to

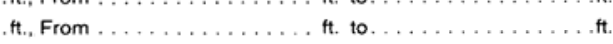

$20 \ldots . .$. ft. to ft. to

$55 \ldots . . . . .10$ From

$\begin{array}{ll}\text { Ht., From } \\ \mathrm{X} \text { Bentonite } & 4 \text { Other }\end{array}$

ft. to

ft. to

ft. to. ......... ft., From......

10 Livestock pens

..t. to to ........t. th.

7 Pit privy

11 Fuel storage

14 Abandoned water well

12 Fertilizer storage

15 Oil well/Gas well

13 Insecticide storage

How many feet? $\quad 150$

16 Other (specity below)

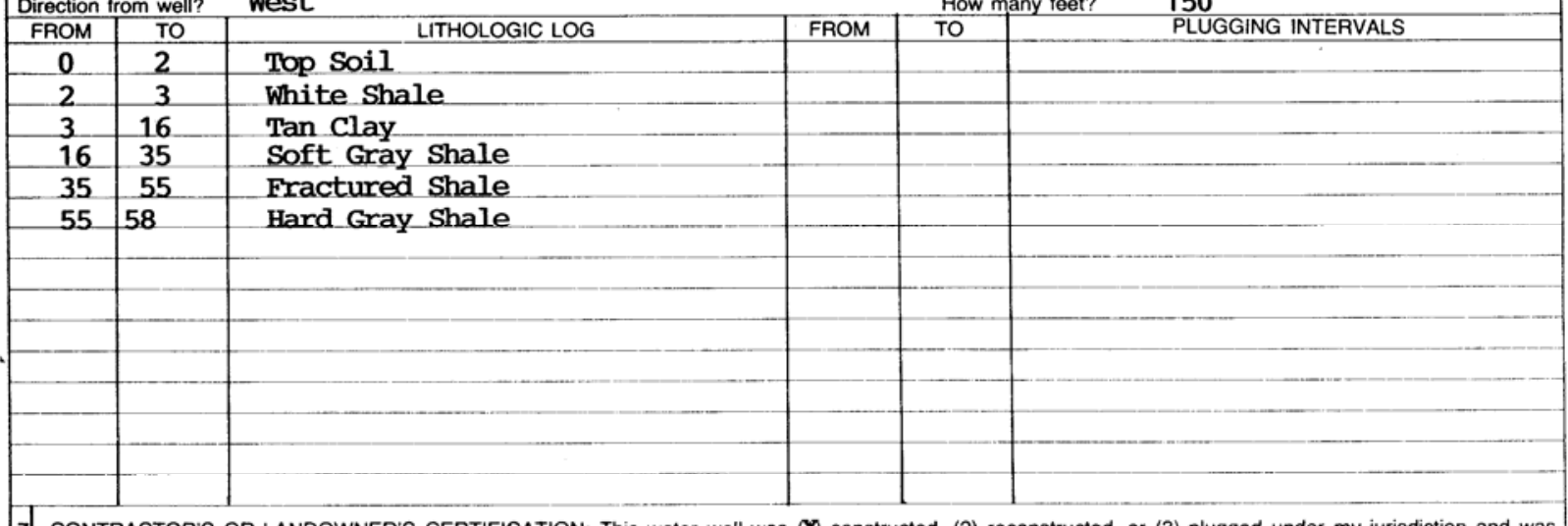

7 CONTRACTOR'S OR LANDOWNER'S CERTIFICATION: This water well was constructed, (2) reconstructed, or (3) plugged under my jurisdiction and was

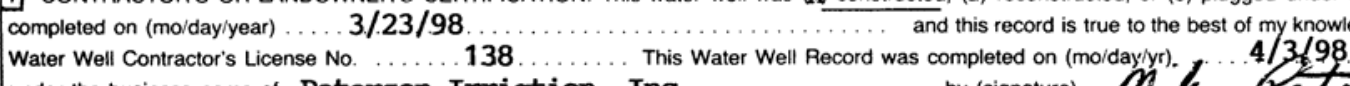
under the business name of Peterson Irrigtion, Inc.

by (signature) Plece Reters

INSTRUCTIONS: Use typewriter or ball point pen. PLEASE PRESS FIRMLY and PRINT clearly. Please fill in blanks, underline or circle the correct answers. Send top three copies to Kansas Department of Heakth and Environment, Bureau of Water, Topeka, Kansas 66620-0001. Telephone: 913-296-5545. Send one to WATER WELL. OWNER and retain one tor your records. 


\section{DW63}

USE IYPEWRITER OR BALL

PRINT CLEARLY.

WATER WELL RECORD

Konsas Department of Health and Environment-Division of Environment

(Water well Contractors)

Topeka, Kansas 66620

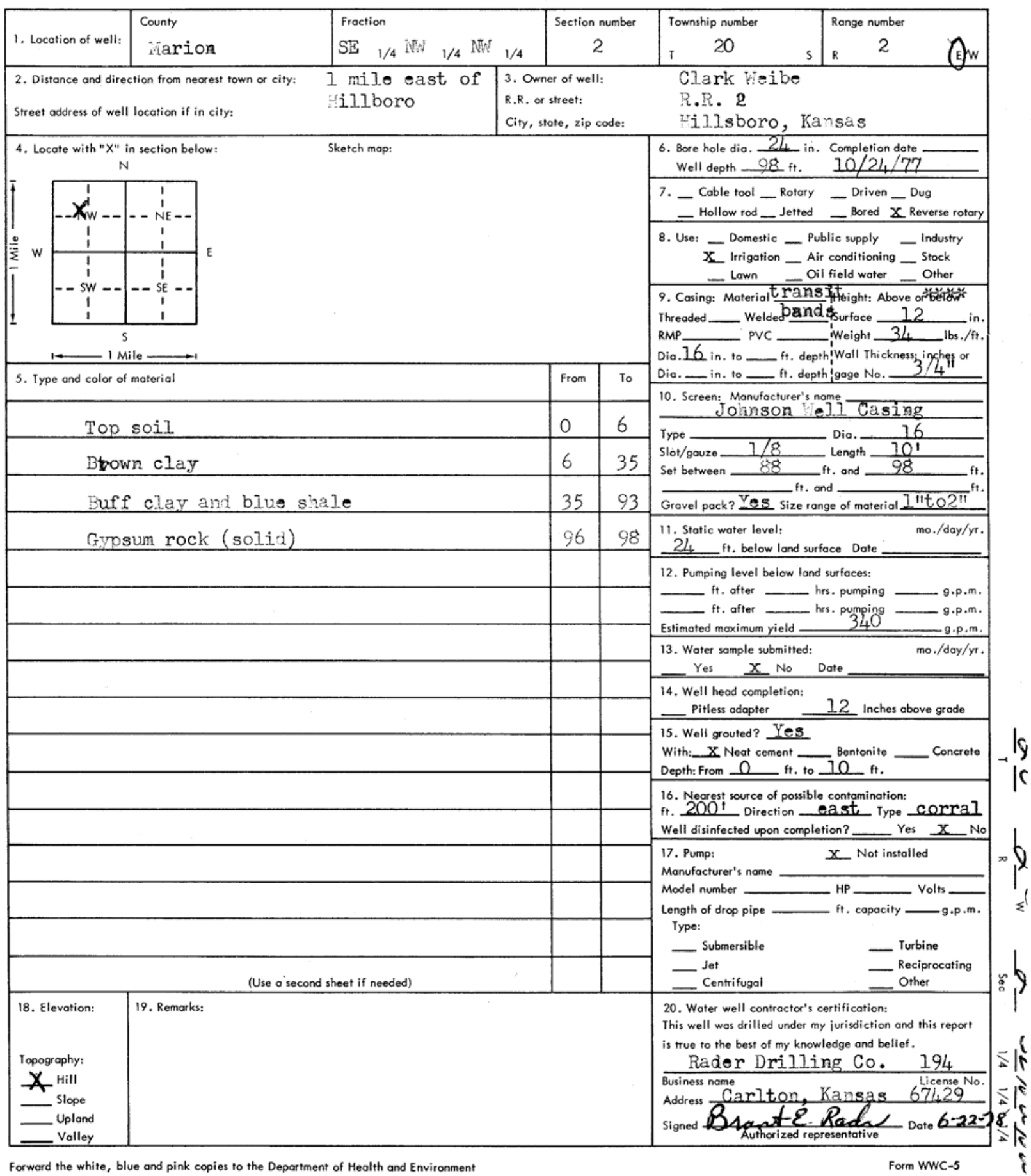




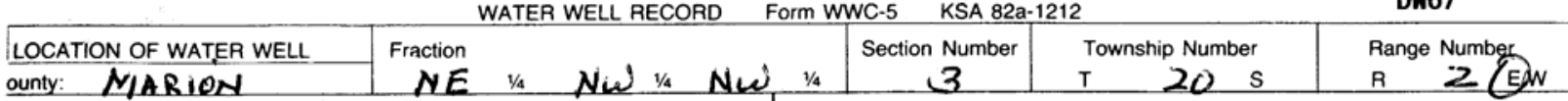

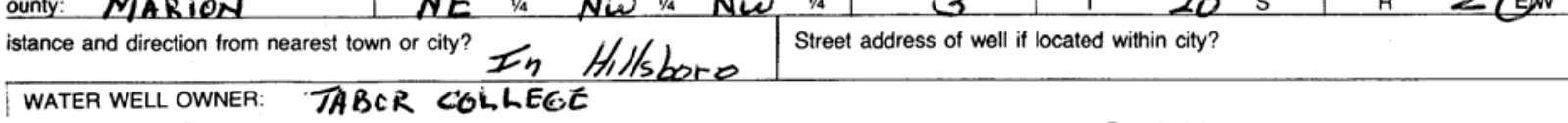

\section{R\#, St. Address, Box \# :}

ity, State, ZIP Code : H. ils bewes; Kins

DEPTH OF COMPLETED WELL.. . 9. .....ft. Bore Hole Diameter....

Jell Water to be used as:

1 Domestic 3 Feedlot

2 Irrigation 4 Industrial

lell's static water level ...37.

ump Test Data

st. Yield 5 Public water supply 6 Oil field water supply

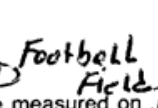
$12 \%$. in. to
8 Air conditioning
9 Dewatering ...ft. below land surface measured on

0 Observation well Well water was. . . 40... ft. after Well water was

TYPE OF BLANK CASING USED:
1 Steel
2 PVCS
$4 \mathrm{ABS}$

3 RMP (SR)

ilank casing dia ....8

iasing height above land surface. EEN OR PERFORATION MATERIAL:
1 Steel
3 Stainless steel
2 Brass
4 Galvanized steel

icreen or Perforation Openings Are:

1 Continuous slot

2 Louvered shutter

3 Mill slot

Pen-Poration Dia ........ in to . 88

5 Wrought iron

6 Asbestos-Cement

194 . Fibergass

8 Concrete tile

9 Other (specify below)

Application Number:

creen-Pertorated Intervals: From. $48.8 \%$. . . . Dia

5 Fiberglass

6 Concrete tile in. to.

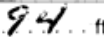

11 Injection well

12 Other (Specify below)

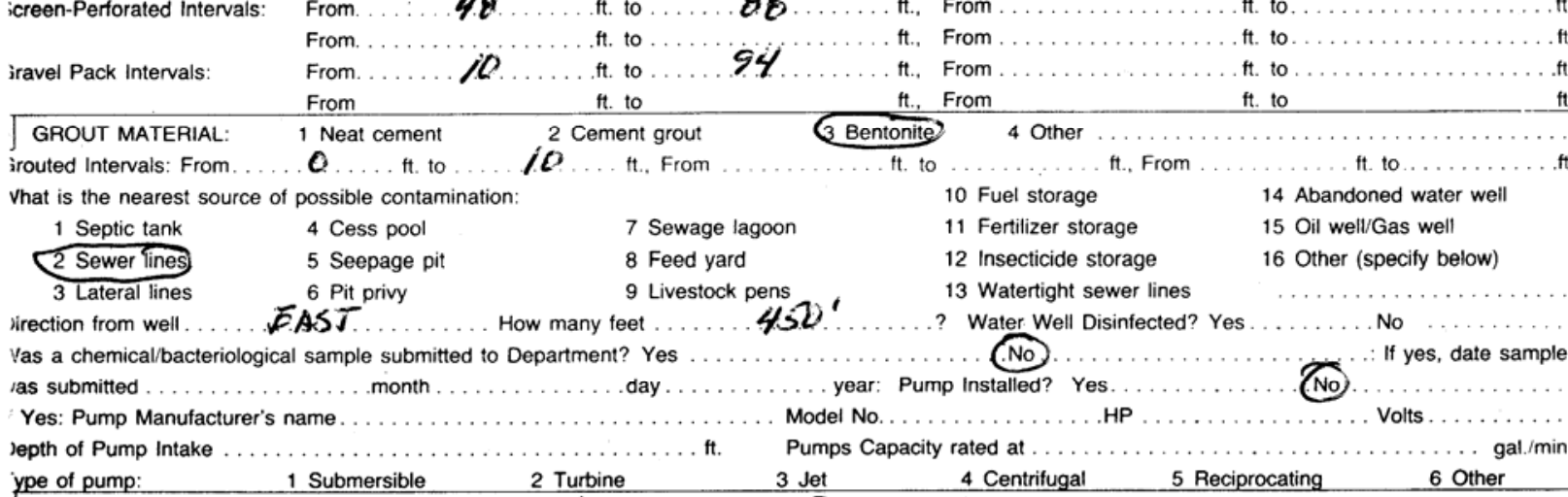
CONTRACTOR'S OR LANDOWNER'S CERTIFICATION This water well was (1) constructed, (2) reconstructed, or (3) plugged under my jurisdiction and wa:

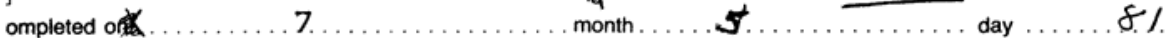

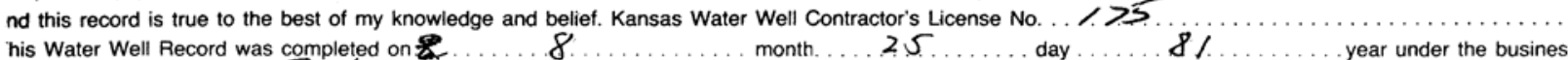
iame of LOCATE WELL'S LOCATION WITH AN " $X$ " IN SECTION BOX:

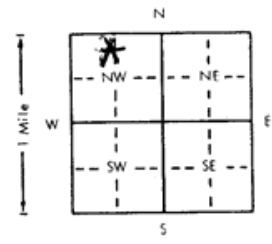

ELEVATION

\begin{tabular}{|c|c|}
\hline FROM & Th \\
\hline 0 & 5 \\
\hline 5 & 10 \\
\hline 10 & 2 \\
\hline 25 & 3 \\
\hline 35 & 4 \\
\hline 40 & 5 \\
\hline 50 & 5 \\
\hline 56 & 3 \\
58 & 6 \\
\hline 65 & 7 \\
25 & 8 \\
\hline
\end{tabular}
The by (signature)

5 Gauzed wrapped

10 Asbestos-cement

12 None used (open hole)

9 Drilled holes

11 None (open hole)

ther (specify)

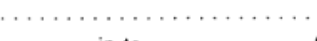
Torch cut

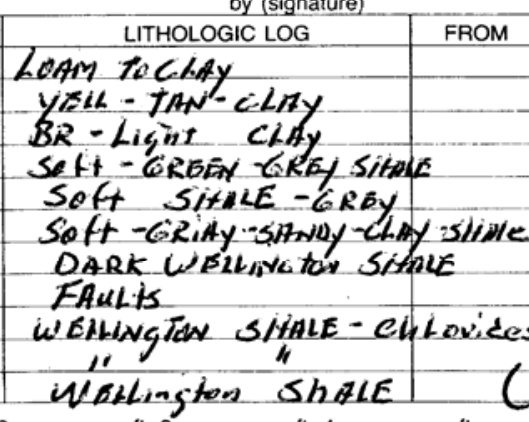

(Fnultel from $75^{\circ}$ to $0^{\circ}$ )

Jepth(s) Groundwater Encountered 1.

ft. 2 . ft. 3 . ft. 4 Use a second sheet if needed NSTRUCTIONS: Use typewriter or ball point pen, please press firmly and PRINT clearly. Please fill in blanks, underline or circle the correct answers. Send top three opies to Kansas Department of Health and Environment, Division of Environment, Water Well Contractors, Topeka, KS 66620. Send one to WATER WELL OWNER and etain one for your records. 
DW95

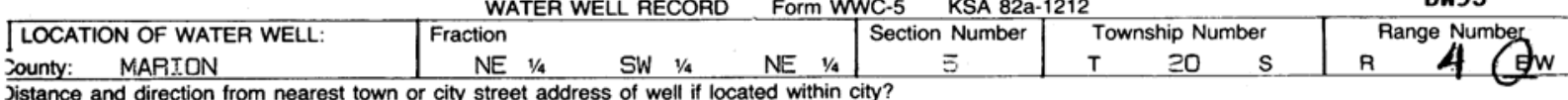
Jistance and direction from nearest town or city street address of well if located within city? 1014 Esst Melvin

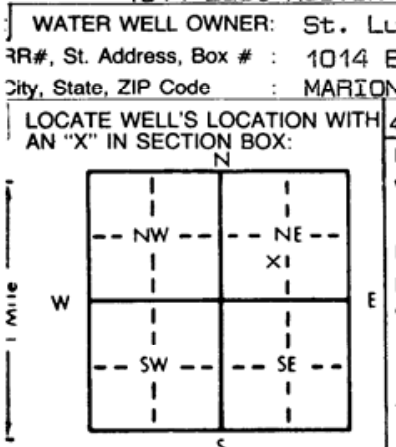

;] TYPE OF BLANK CASING USED:

1 Steel 3 RMP (SR)

2 PVC

4 ABS

Casing height above land surface............. 18

TYPE OF SCREEN OR PERFORATION MATERIAL:
1 Steel
2 Brass
3 Stainless steel
4 Galvanized steel

SCREEN OR PERFORATION OPENINGS ARE:

1 Continuous slot 3 Mill slot

2 Louvered shutter 4 Key punched

SCREEN-PERFORATED INTERVALS: From.

From.

From

From......... th. to

GRAVEL PACK INTERVALS:

From

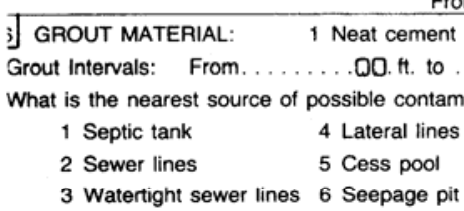

\section{Pit privy \\ 8 Sewage lagoon \\ 9 Feedyard}

2 Cement grout

77. ft., From

ft. to

3 Bentonite

113.. . H. ELEVATION:
Board of Agriculture, Division of Water Resources Application Number:
.... ft. to. .

51. ft. 2.

below land sutace measued on moft. $3 . \ldots \ldots \ldots \ldots \ldots$ ft.

face measured on mo/day/yr

ft. after ........ hours pumping ........ gpm

ft. after hours pumping ....... $75 \ldots$ ft., and. . . . . . . . . . .

11 Injection well

12 Other (Specify below)

Beck . Up. Wațer Suppely

If yes, mo/day/yr sample was sub. No ...........; If yes, mo/day/yr sar

Water Well Disinfected? Yes CASING JOINTS: Glued $\times x . \ldots$ Clamped . . . . . . Welded . . . . . . . . . . . . . Threaded. . . . . . . . . . . . 9 Other (specify below)

$\ldots \ldots$ ..... in to in. to ............. Dia . . . . 7 PVC 10 Asbestos-cement
8 RMP (SR)
11 Other (specify)

9 ABS

12 None used (open hole)

8 Saw cut 11 None (open hole)

9 Drilled holes

10 Other (specify)

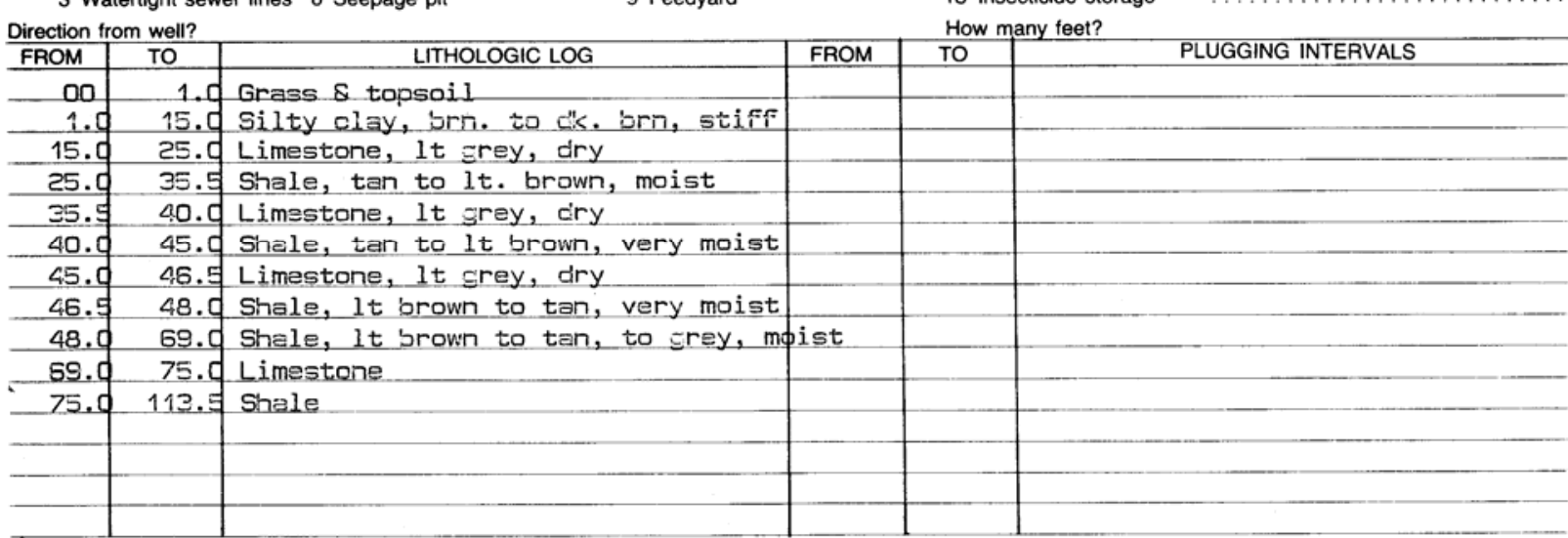

77 CONTRACTOR'S OR LANDOWNER'S CERTIFICATION: This water well was (1) constructed, (2) reconstructed, or (3) plugged under my jurisdiction and was

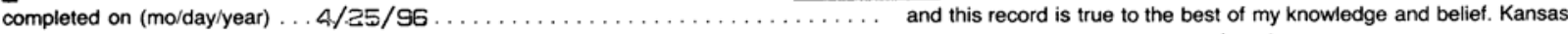

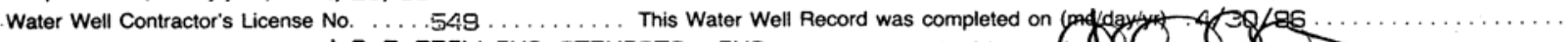
under the business name of $J \& R$ DRILLING SERVICES, INC. 


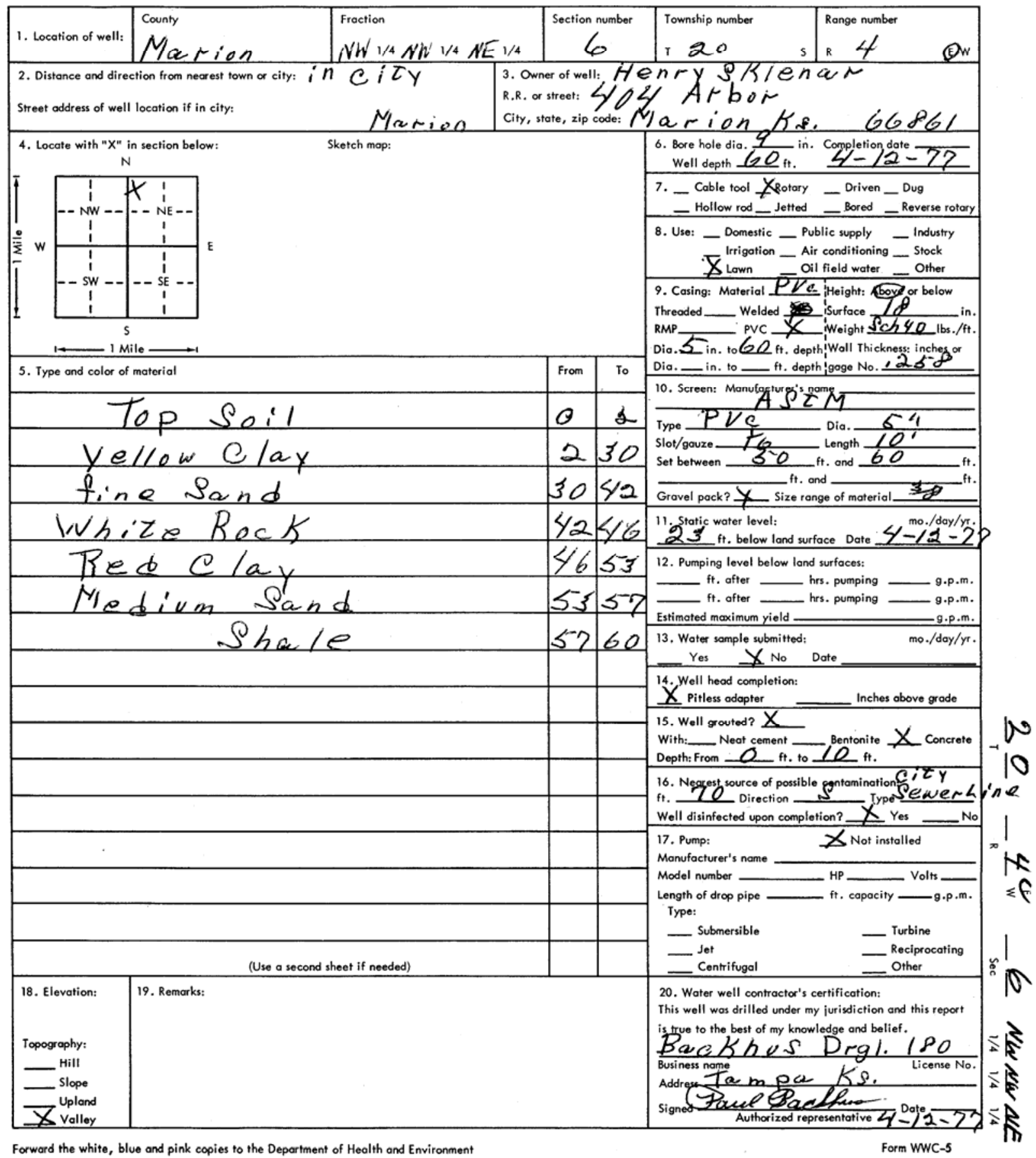


DW99

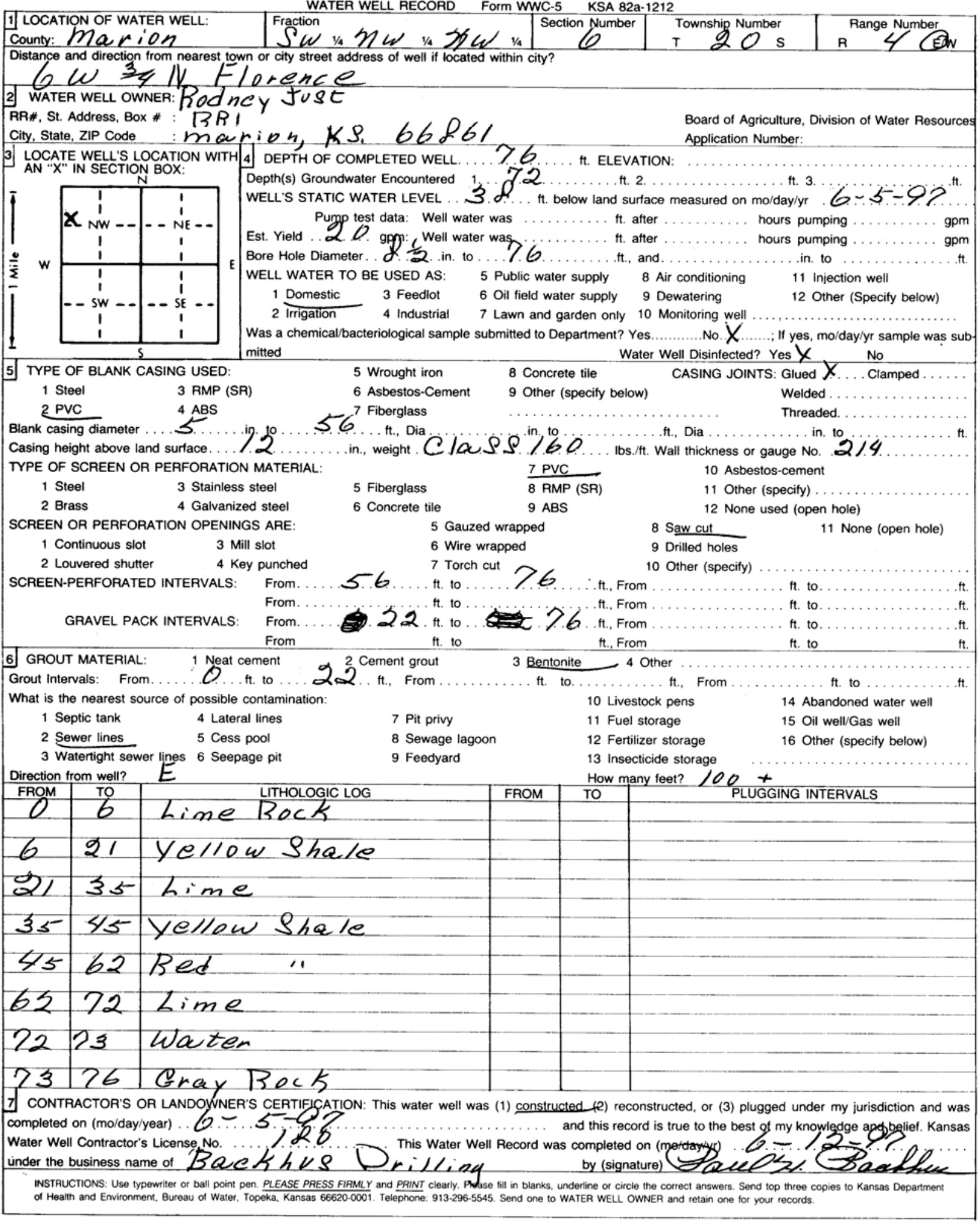




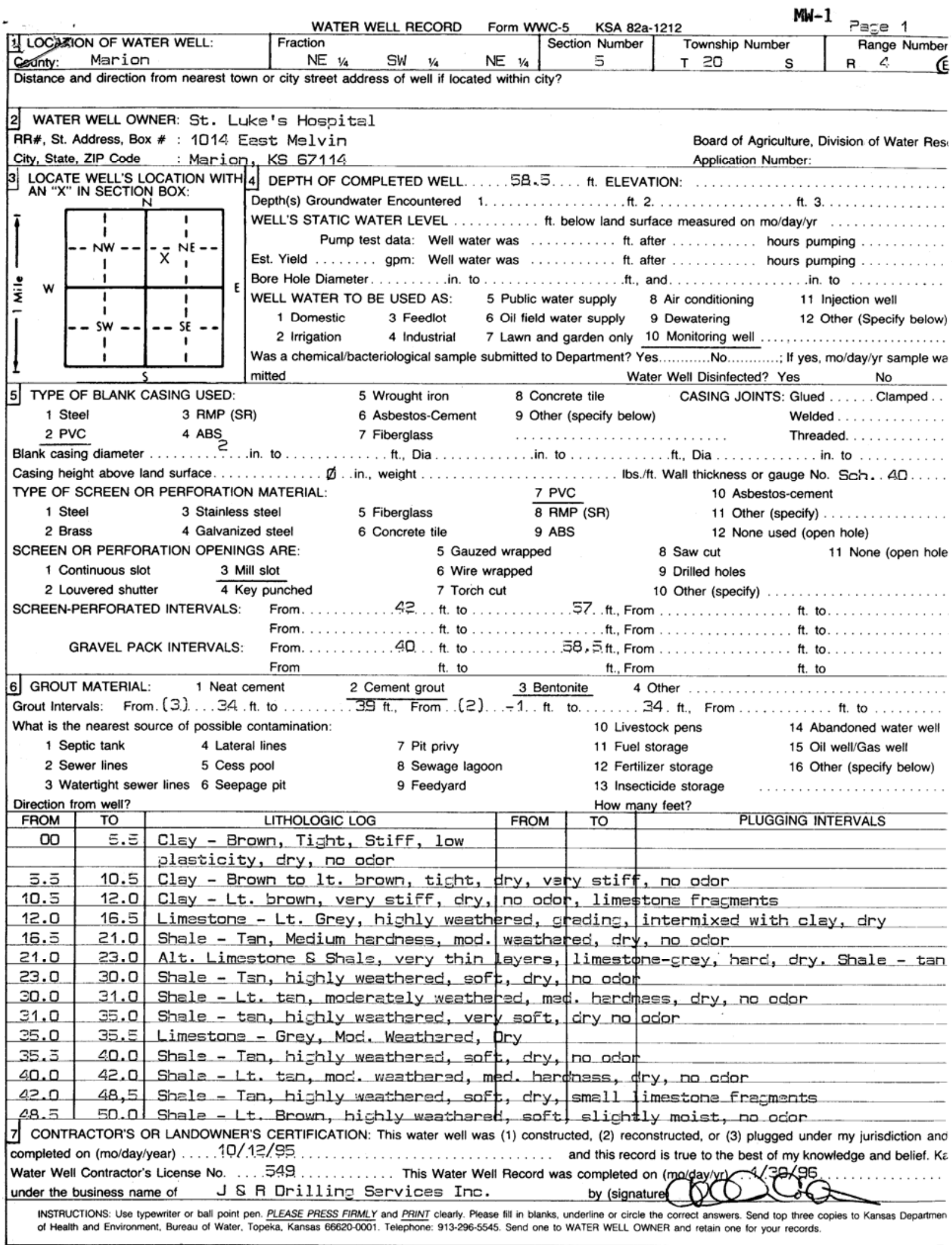




\begin{tabular}{|c|c|c|c|c|c|}
\hline 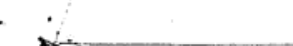 & WATER WELL RECORD & Forr & KSA 82 & $M W-1$ & Dage ? \\
\hline NEDF WATER WELL: & Fraction & & Section Number & Township Number & Range Number \\
\hline Marion & SW $1 / 4$ & $\mathrm{NE} 1 / 4$ & 5 & 20 & $\mathrm{R}$ \\
\hline
\end{tabular}
and direction from nearest town or city street address of well if located within city?

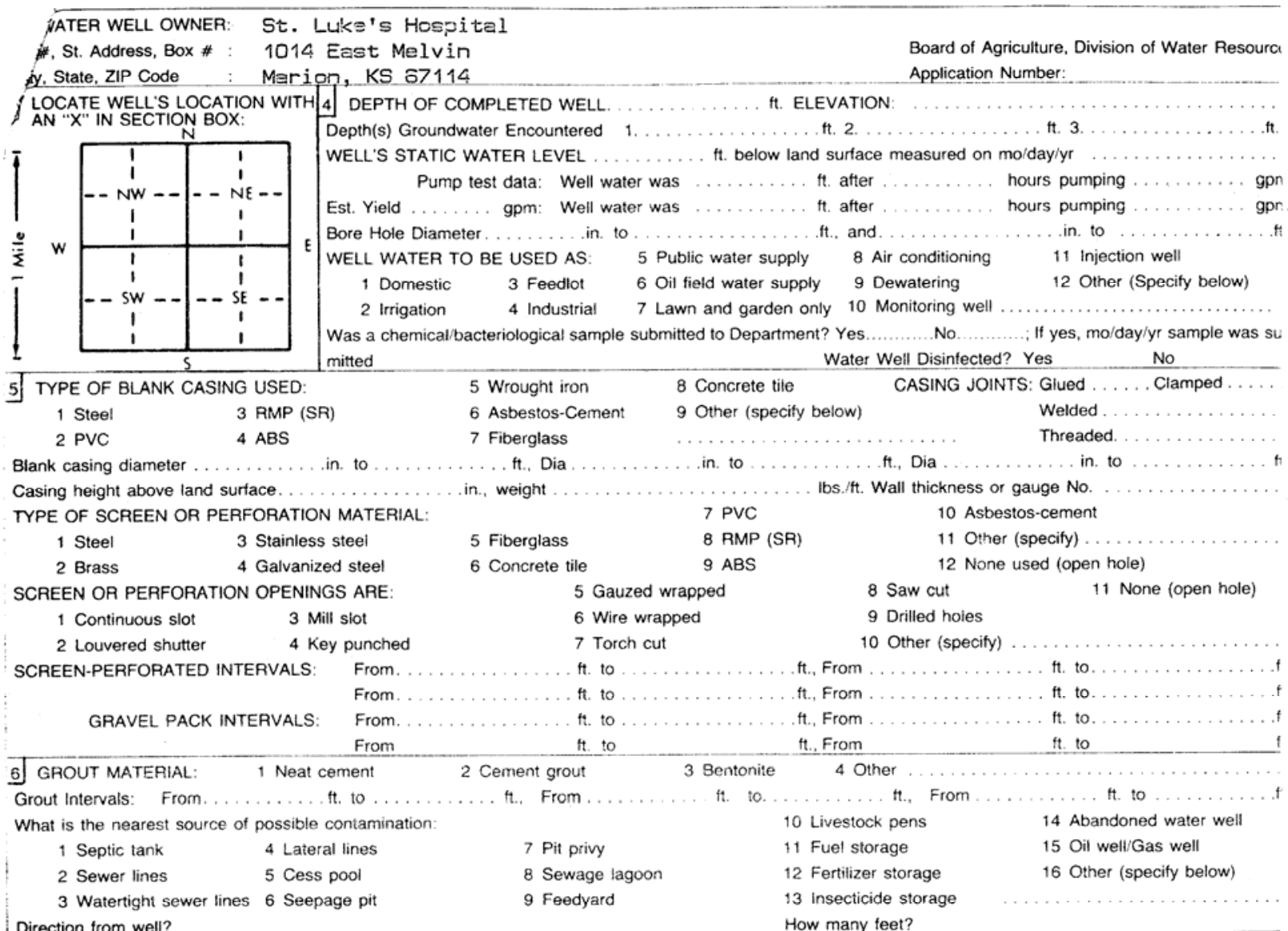

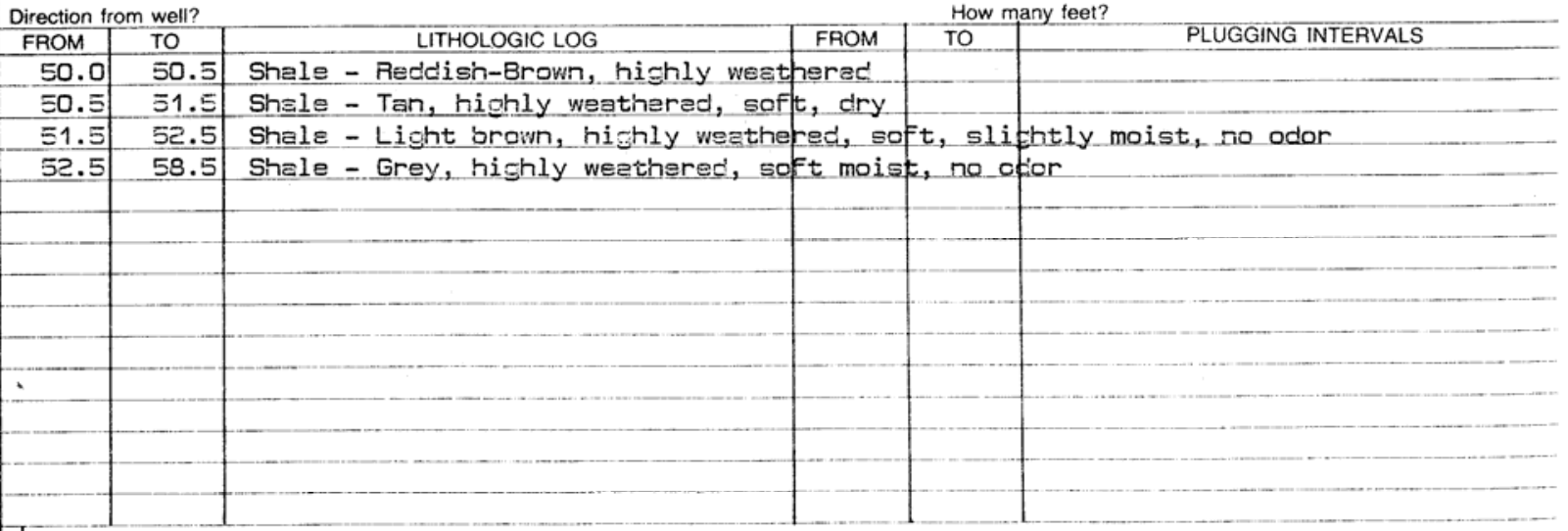

7 CONTRACTOR'S OR LANDOWNER'S CERTIFICATION: This water well was (1) constructed, (2) reconstructed, or (3) plugged under my jurisdiction and w: completed on (mo/day/year) 


\section{Appendix B:}

\section{Geology of the Canada Area, Marion County}




\section{Appendix B:}

\section{Geology of the Canada Area, Marion County}

\section{B.1 Geologic Data}

Subsurface geologic data are generally sought from well registration forms and drilling records of test holes and irrigation, domestic, monitoring, and municipal wells. Irrigation is not a general farming practice in the region, and the Kansas Geologic Survey (KGS) has not drilled test holes in the study area. Therefore, the only available lithologic logs are in records of domestic, municipal, and monitoring wells. The water well record (WWC-5 form) is a standard form that all licensed water well contractors are required to complete for each water well drilled in Kansas. The available water well log data were examined critically for completeness and consistency. Most log descriptions were too brief to determine the lithologic units accurately. Although several lithologic logs from WWC-5 forms exist for water wells in Canada and the surrounding area, their inconsistent quality makes interpretation and correlation of the geologic units difficult, if not impossible. A lack of consistency in the quality of lithologic logs in Marion County is a recognized problem (Chaffee 1988). In addition, well locations are described in general terms (township and range) and persistenty identify the individual domestic wells within the town inadequately. No well records exist for most domestic wells, and the names and addresses of the original listed owners frequently do not match those of any current well owner.

Documents consulted include reports published by the U.S. Geological Survey (USGS) and the USDA Soil Conservation Service, open-file reports of the KGS, and an environmental impact statement from the U.S. Army Corps of Engineers (USACE). Information on the regional geology and physiography was also obtained directly from the KGS World Wide Web site at http://www.kgs.ukans.edu. Because of the absence of reliable geologic logs of boreholes in central Marion County, the surface geologic maps generated by the USGS (Byrne et al. 1959) provided the only reliable source of geologic data. This information was used to extrapolate the subsurface geology. The established stratigraphic column (giving the sequence and thicknesses of the shales and limestones) was extrapolated from the nearest outcropping geologic sequence by using a regional dip and strike computed from the intersection of the contact with the topography. The computed apparent dip in the direction of each segment of the cross section was then used to extend the surface outcrop section to the location and depth of the drillers' logs. Because the log descriptions bear little resemblance to the actual lithologies, validation of the 
interpretive geologic sections relied on correlation between (1) the locations of changes in major lithologic type as described in the drillers' logs and (2) the locations of stratigraphic contacts predicted from the USGS surface geology (Figure B.1; Byrne et al. 1959).

\section{B.2 Geologic Setting}

Marion County is largely within the Flint Hills region of east central Kansas. The county includes a small area of the Smoky Hills region in the northwest and the Wellington-McPherson Lowlands in the southwest. The eastern portion of the county is characteristic of the Flint Hills region, with well-dissected topography, deeply incised valleys, thin soils, and many limestone outcrops. The western part of the county is predominantly shale beds containing a few relatively thin, soft limestones. Such units tend to form a gently rolling to nearly flat topography that is amenable to agriculture. Few good natural rock outcrops are present in this area. The major drainage from Marion County is southeastward via the Cottonwood River, forming the upper reaches of the Neosho River drainage basin. The Cottonwood River was dammed in 1968 to form Marion Lake in the central part of the county (for location see Figure B.2). The 10,800-acre lake is used for flood control, water quality control, water supply, recreation, and fish and wildlife conservation (Jordan and Hart 1985).

The surface geology of Marion County has been described by Byrne et al. (1959). Figure B.2 illustrates the generalized surface geology. The bedrock of Marion County is largely Lower Permian shale with interbedded chalky and cherty limestone. These beds dip gently westward with a slope of about 15-20 ft/mi (O'Connor and Chaffee 1992). Limestones and shales of the Chase Group, exposed throughout the eastern part of the county, are the oldest Lower Permian rocks present. In Marion County, the Chase Group is overlain by about $450 \mathrm{ft}$ of Wellington Formation shales, part of the Lower Permian Sumner Group. Lower Cretaceous shale and sandstone unconformably overlie Permian strata in the northwest corner of the county. Unconsolidated Cenozoic sediments of late Tertiary and Pleistocene age also unconformably overlie the Permian beds in the southwest part of the county (Figure B.2).

The oldest rocks that outcrop in the central part of Marion County in the vicinity of Canada are from the Lower Permian Chase Group, made up of about $300 \mathrm{ft}$ of limestone and dolomitic limestone alternating with shale in repetitive sequences called cyclotherms (Terman and Aber 1994). These cyclotherms are the products of repeated transgressions and regressions by shallow seas over a nearly flat depositional surface in the early Permian. Younger Wellington 
Formation shales of the Sumner Group conformably overlie the Chase Group and outcrop throughout the central and western region of the county, including the area surrounding the town of Canada. These rocks represent a change to more continental, extremely arid conditions during the Permian. The shale is variously colored gray, green, and red and contains thin beds of limestone and gypsum.

The Quaternary system is represented in the vicinity of Canada by scattered thin silt and sand deposits that may be erosional remnants of an eastward extension of the Early Pleistocene McPherson valley alluvium. Byrne et al. (1959) called these deposits the Sanborn Formation. Younger Pleistocene terrace deposits occur along major streams, and the valley floors are filled with Recent alluvium. Alluvial deposits are thickest in the valleys of the larger streams and are known to exceed a thickness of $30 \mathrm{ft}$ on the Cottonwood River at the town of Marion (USACE 1974). Soils in the central part of the county are derived from the weathering of limestone, cherty limestone, calcareous and noncalcareous shale, alluvium, and colluvium.

The location of an interpretive regional cross section passing through Canada is shown in Figure B.3. Stratigraphic relationships within the shallow bedrock, the elevation of the ground surface, and relief on the bedrock surface are illustrated schematically in west-to-east section A-A' (Figure B.4). Copies of the drillers' logs from which this section was constructed are in Appendix A.

Cross section A-A' (Figure B.4) shows that the Permian formations dip from east to west. The strike and dip can be computed by transposing the geologic contacts from the geologic map (Byrne et al. 1959) to the 1:24,000-scale topographic base (USGS 1985) and measuring the elevation of a single stratigraphic horizon at three locations. On the basis of the intersection of the top of the Nolans limestone with the topography, the beds strike $\mathrm{N} 30^{\circ} \mathrm{W}$ and dip S $60^{\circ} \mathrm{W}$ at an angle of $0.2^{\circ}$ (about $17 \mathrm{ft} / \mathrm{mi}$ ) in central Marion County. Note that the vertical exaggeration of the cross section (1) makes the westward slope of the bedrock strata appear to be far steeper than the shallow angle actually observed in the field and (2) enhances subtle changes in the surface topography. This reconstruction indicates that the shallow geologic section at Canada should be composed of soil 2-3 ft thick, developed from loess and weathered shale at the surface and overlying weathered Wellington shale. The shale weathers to clay that has been variously recorded in drillers' logs to depths of 11-42 ft BGL. The top of the Nolans Formation (Herington limestone member) is projected to occur at a depth of approximately $25 \mathrm{ft}$ BGL. This unit is routinely described as "blue shale” in drillers' logs at this depth. However, the KDHE reported 
refusal of the conductivity probe at a hard layer postulated to be limestone at $13 \mathrm{ft}$ BGL (KDHE 1999a). The stratigraphic data available suggest that this postulated limestone might be one of the relatively thin dolomitic limestones known to occur within the Pearl shale member at the base of the Wellington Formation. The top of the underlying Odell shale, projected from outcrop and drill holes to the east, should occur at about $65 \mathrm{ft}$ BGL.

\section{B.3 Hydrogeology}

The principal aquifers in the Flint Hills are the Nolans, Winfield, and Barneston limestones of the Lower Permian Chase Group. The Nolans and Winfield limestones are shown on cross section A-A' (Figure B.4). Sinkholes are common where these limestones crop out in the uplands, and springs emerge where the aquifers are exposed in valleys and stream channels. Most wells produce from 10 to $100 \mathrm{gpm}$, with some producing as much as 500 gpm (Terman and Aber 1994). Relatively impermeable shale units separate the bedrock aquifers, setting up confined conditions in which each aquifer might have a different hydraulic head.

Where the Wellington Formation overlies the Chase Group limestones to the west of the Flint Hills, groundwater is also produced from fractured shale and several thin beds of impure limestone within the Wellington. The formation yields small quantities of highly mineralized water to many stock and domestic wells. Moderately large water supplies of highly mineralized but usable groundwater are available from solution zones in gypsum beds.

Groundwater generally flows from east to west in the limestone aquifers. Recharge takes place where the aquifers outcrop to the east, and water moves down the regional bedrock dip toward the west. However, local variations in the regional groundwater flow direction are common because of lowering of the potentiometric surface in the limestone aquifers by seepage along valleys and stream channels (O'Connor and Chaffee 1992). The multiple aquifers, the absence of monitoring wells, and the many local topographic effects preclude construction of meaningful regional potentiometric surfaces from available data for any of the identifiable aquifers. The KGS hydrogeologic database as of 1999 contained no water levels for Marion County.

At Canada, screened intervals for domestic wells for which records are available are 45-60 ft BGL and 54-64 ft BGL in DW22 and DW23, respectively, and an upper zone at 25-35 ft 
BGL and a lower zone at 50-64 ft BGL in DW24 (Figure B.4). Logs for DW22, DW23, and DW24 are in Appendix A. Water is generally produced from solution-jointed limestone units of the Nolans Formation, and the screened depths suggest that more than one of these water-bearing zones are present at Canada. Anecdotal evidence gathered from interviews with local residents suggests that groundwater flows to the east-southeast (KDHE 1998). Topographic features in the area support this conclusion. For example, a local flow direction to the southeast is consistent with the fact that the South Cottonwood River has eroded the Permian rocks to a level of about 1,310-1,320 ft above mean sea level (AMSL) $1.25 \mathrm{mi}$ southeast of Canada, 40-50 ft below the elevation of the ground at Canada. Static water levels recorded when the wells were installed in 1978 and 1979 were 15-18 ft BGL (1,345-1,343 ft AMSL), substantially above the base level (1,310-1,320 ft AMSL) at the South Cottonwood River. Another factor contributing to the southeasterly groundwater flow direction would be mounding of groundwater $1 \mathrm{mi}$ to the north of Canada due to recharge from Marion Lake. 


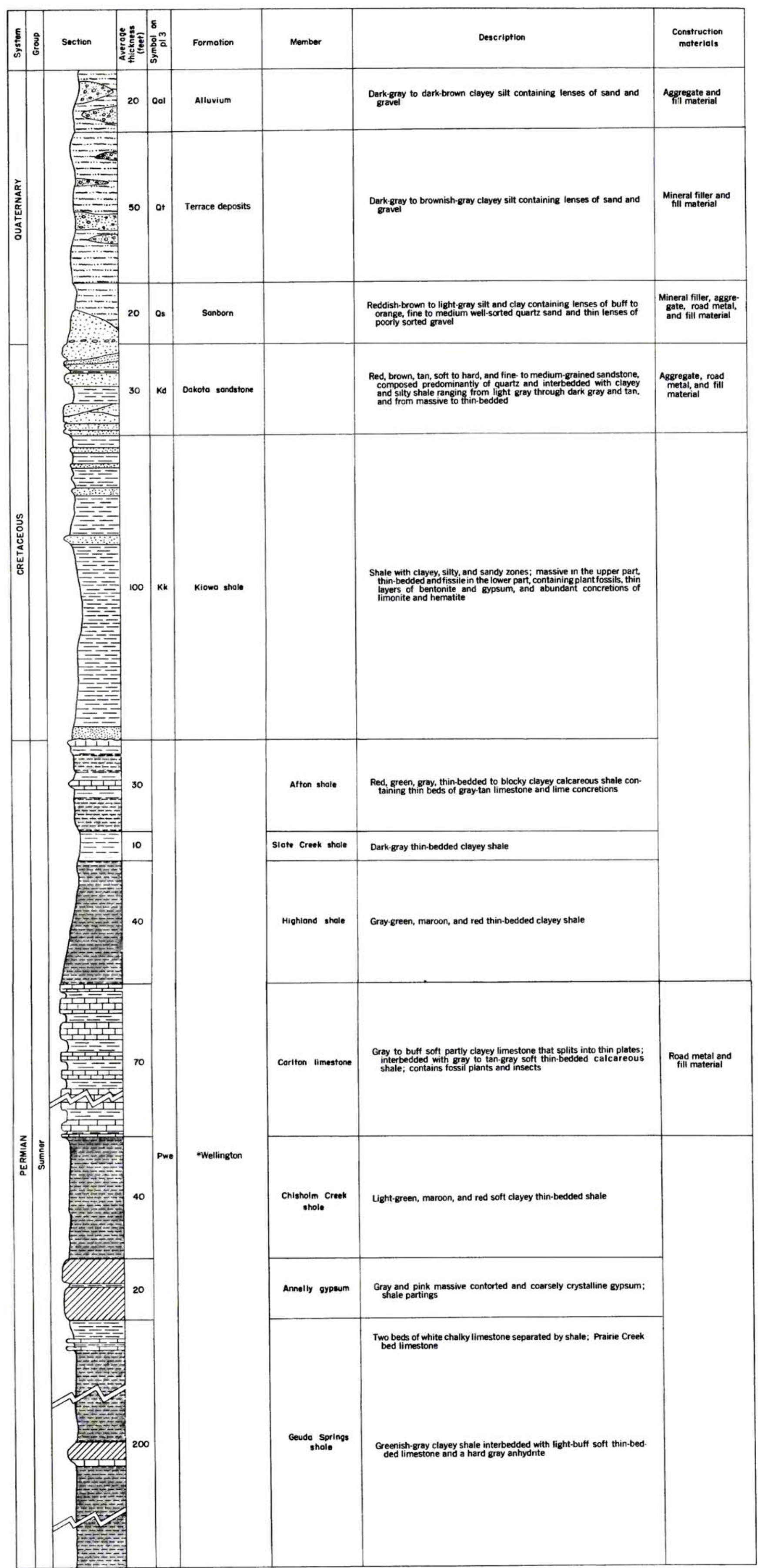

FIGURE B.1 Stratigraphic units that crop out in Marion County, Kansas, and the construction materials of each. Source: Byrne et al. (1959). 


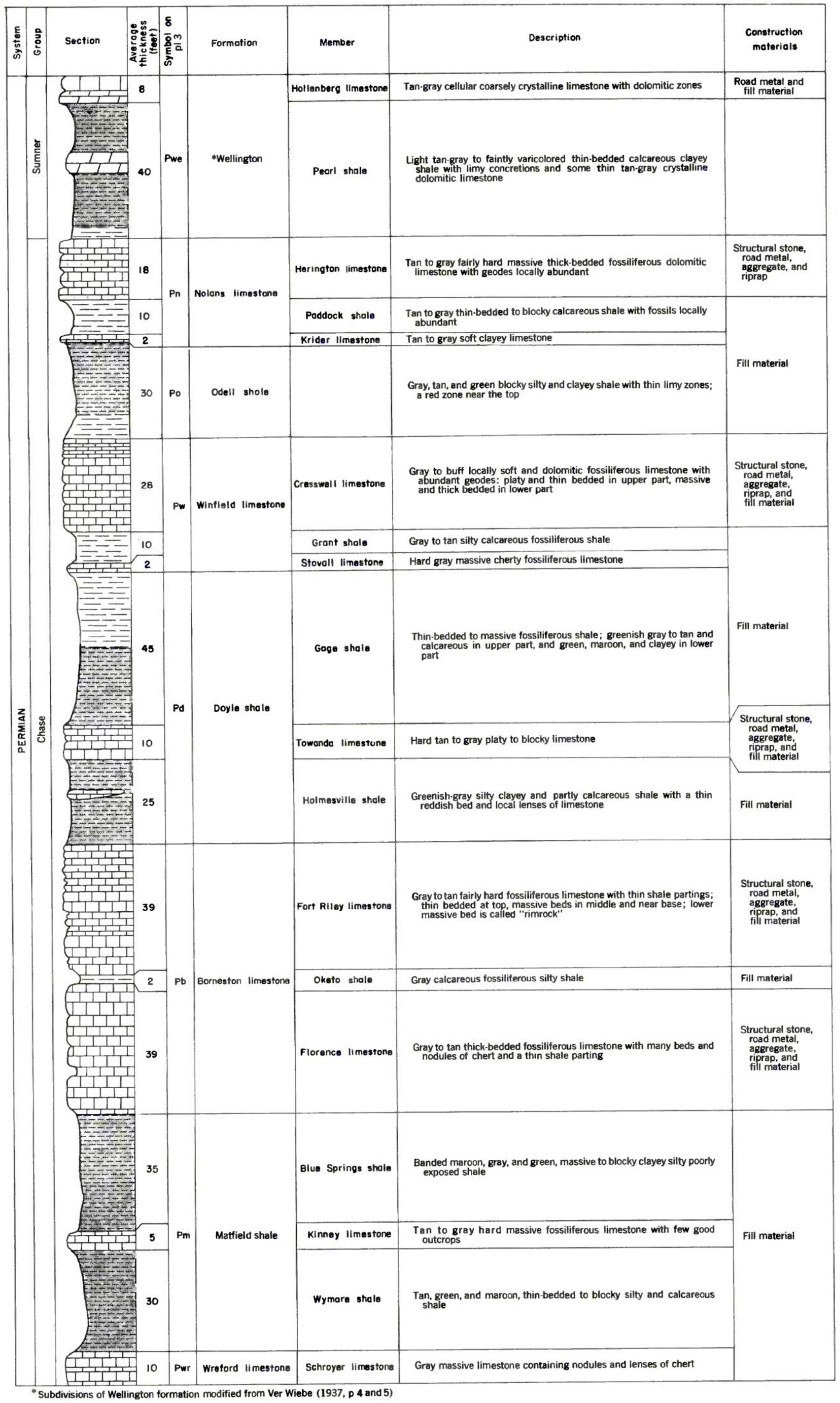




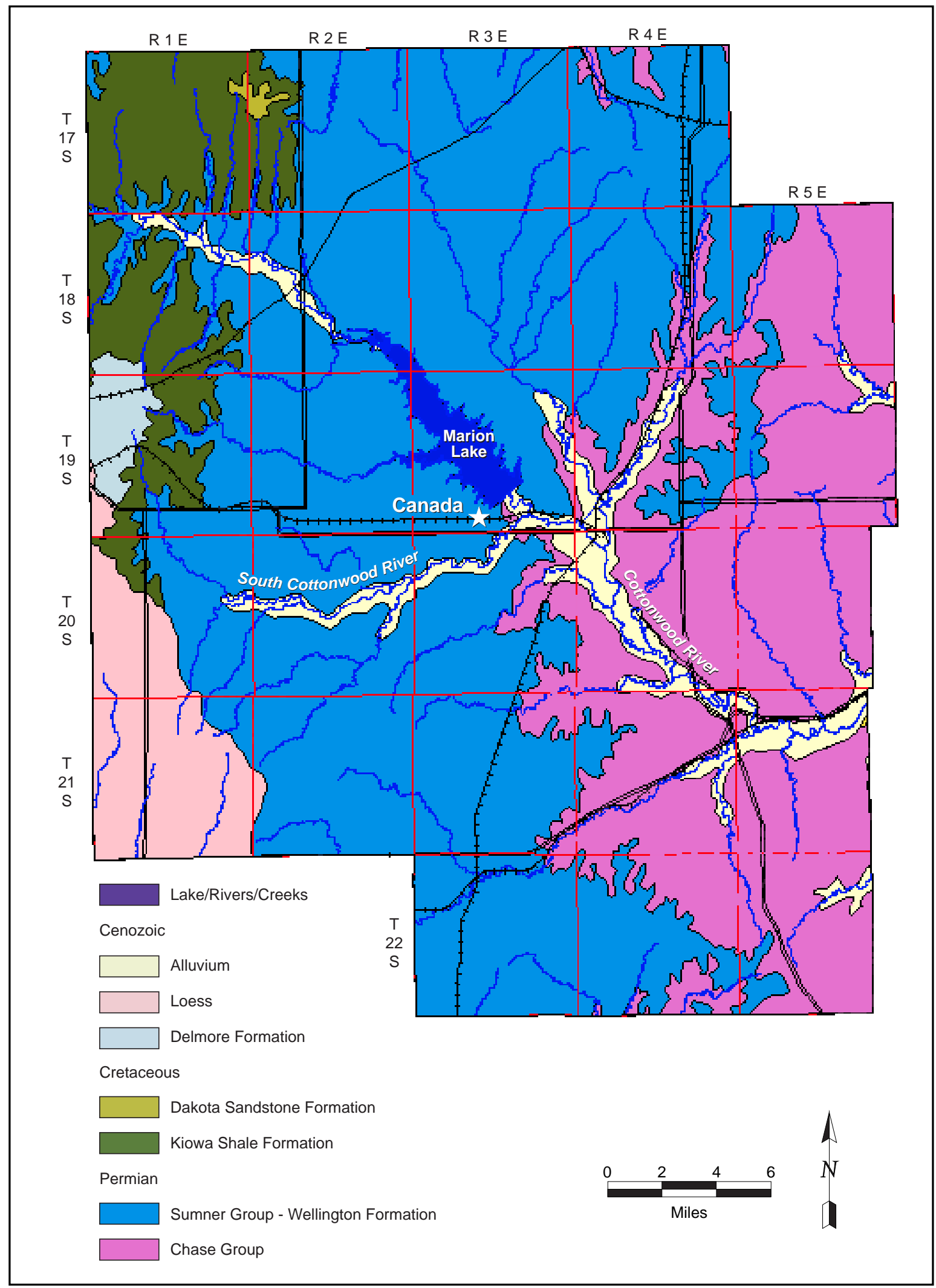

FIGURE B.2 Generalized surface geologic map of Marion County, showing the location of the town of Canada. Source: Byrne et al. (1959). 


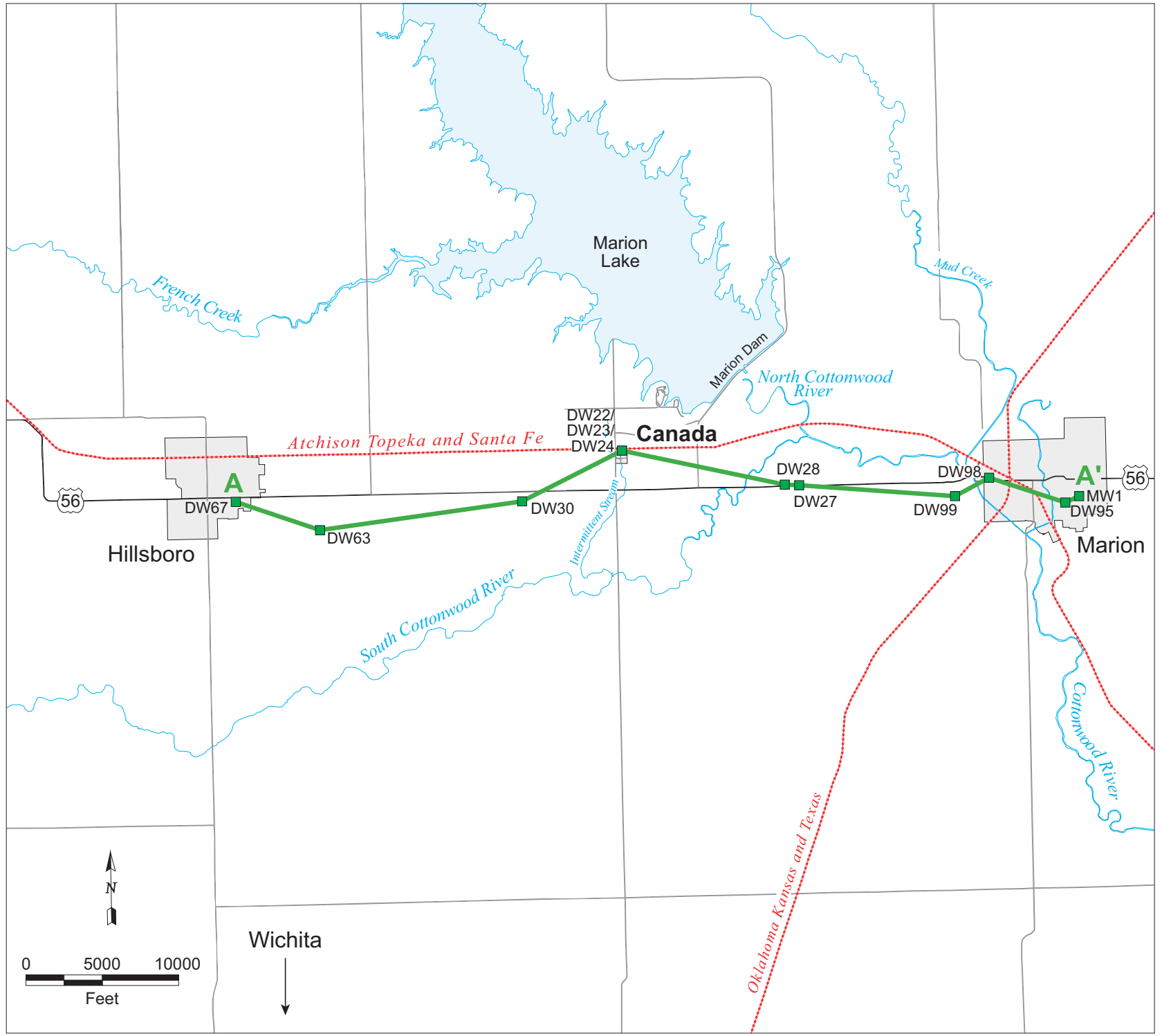

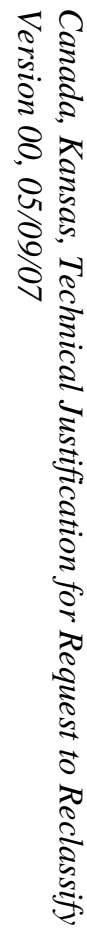

FIGURE B.3 Location of geologic cross section A-A' in central Marion County. 


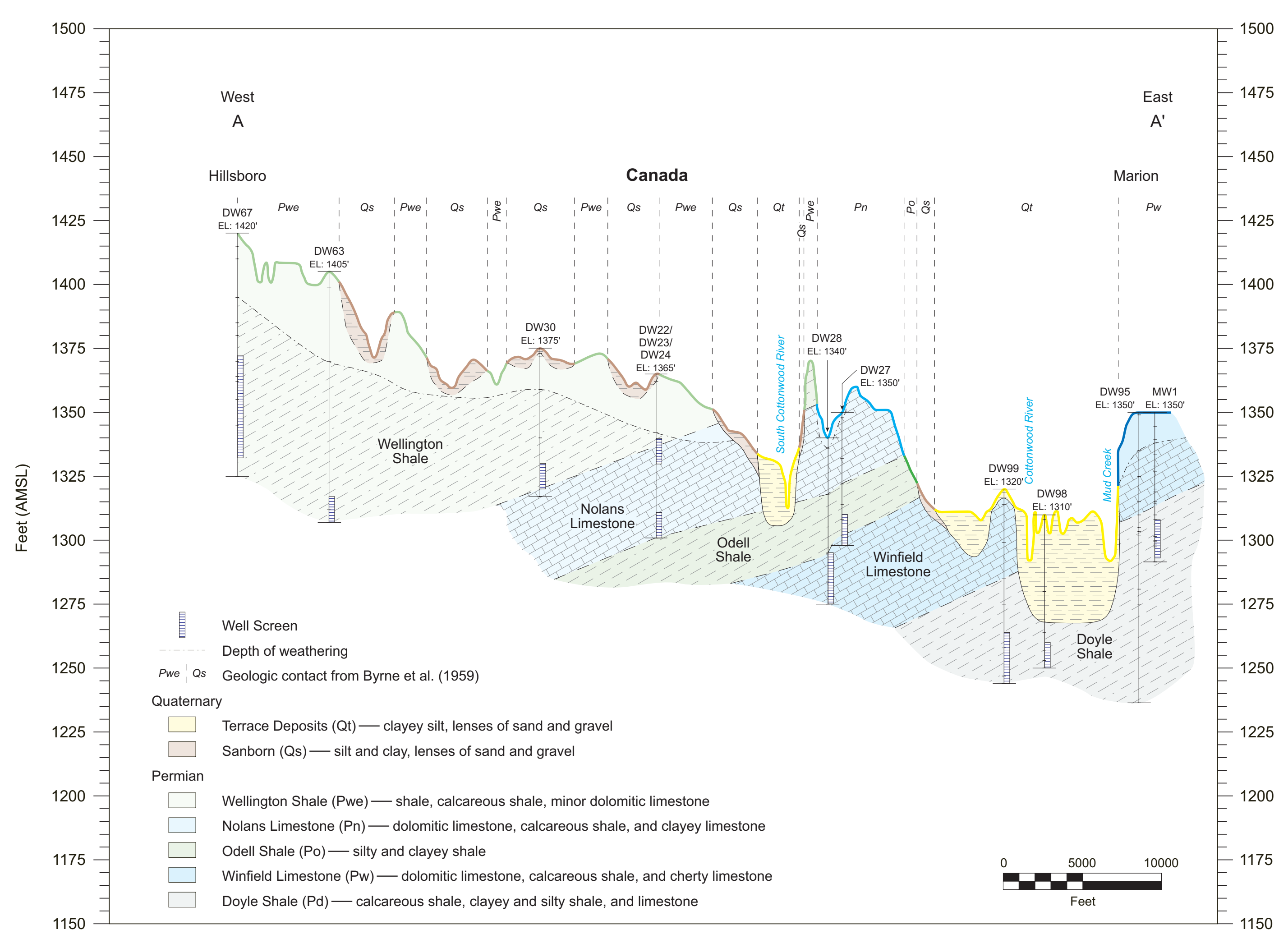

FIGURE B.4 Interpretive west-to-east geologic cross section A-A' (vertically exaggerated), Marion County, Kansas. Source of geologic contacts: Byrne et al. (1959). 


\section{Appendix C:}

Property Records Related to the Former CCC/USDA Facility at Canada 


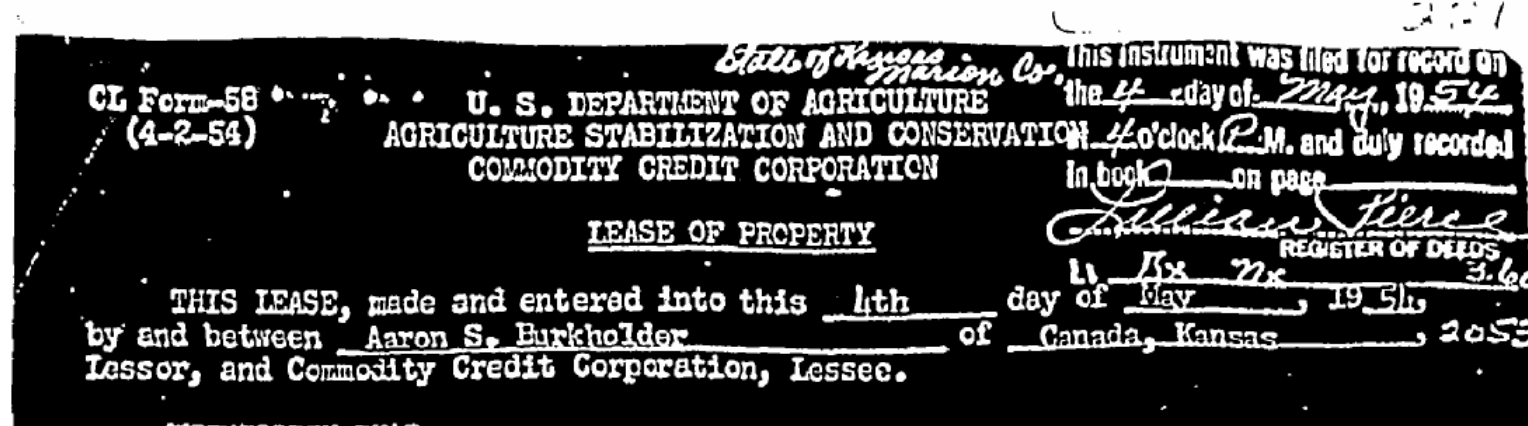

\section{TITNESSETH THAT:}

1. The Iessor leases to the Iessee, and the Iessee hereby leases from the Iessor, upon the terms and conditions horetnarter stated, the follouring described real estate (bereinafter called "property") situated in the County of lofarion and state of Kansas

Lots 7 to 18 inclusive on Blocis 11 in Canada, Kansas

$\because \quad$ containing 1 acres, more or less.

2. The terw of the lease shall te for a pariod of 10 years, commencing

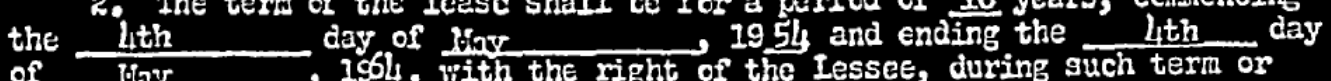
of live 1964, with the right of the Lesses, during such term or any cxtcrsion thereof, to terminate said lease, and liability for any further.

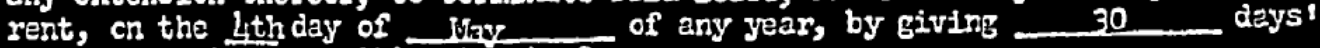
previous notice in rititing to the Icssor.

3. As rent for said property, the Iessee shall pay the Lessor ivinety. and $00 / 10021$ ars ( 40,00 ) per year, such rent to be payable in advance, but to be apportionable in the event the lease is terminated as provided in paragraph 2 hereor: -

4. The Lessor warrants that he is the orner of the property, has the right to give the Iessee possession under this lease, and will, so long as this Iease remains in effect, Harrant and defend the Lessee's possession against ary and all persons whomsoever.

5. 'The Lossce shall have the right, during this lease, to erect storage structures, or facilities, nake alterations, install scalcs, fences, or signs, in or upon the precises hereby leased and, at the expiration of said lease or any renewal or extension thareor or at any time this leasc is in effect, juay remove said storage structures, facilitics, scales, fences, or signs or any part thexeof, whethcr or not such structures, facilities, scales, fences or signs have become legally a fixture.

6. The Isssee shall not assign this lcase vithcut the written consent of the Icsser. The lessce, may, horiever, sublet the structures on the promises leased hereunder, or any one or more of thcm for the turn of the Itase or any part thercof upon such terms and conditions as Iessee may rish tr so sublet.

7. The Lessee, if required by the Lessor, shall upon the expirition of this 7 7. ing at the tiria of cntcring upon the saris under this icase, reasonable anc ordini ing at the and tes and damages by the elerents or by circunstances over which the inssee has riotccritrol cxcepted, Provided, hotrever, That if the Lessor requiras such restoration, the lessor shall give vritten notice thereof to the Lessec 30 days before the termination of the lease. 


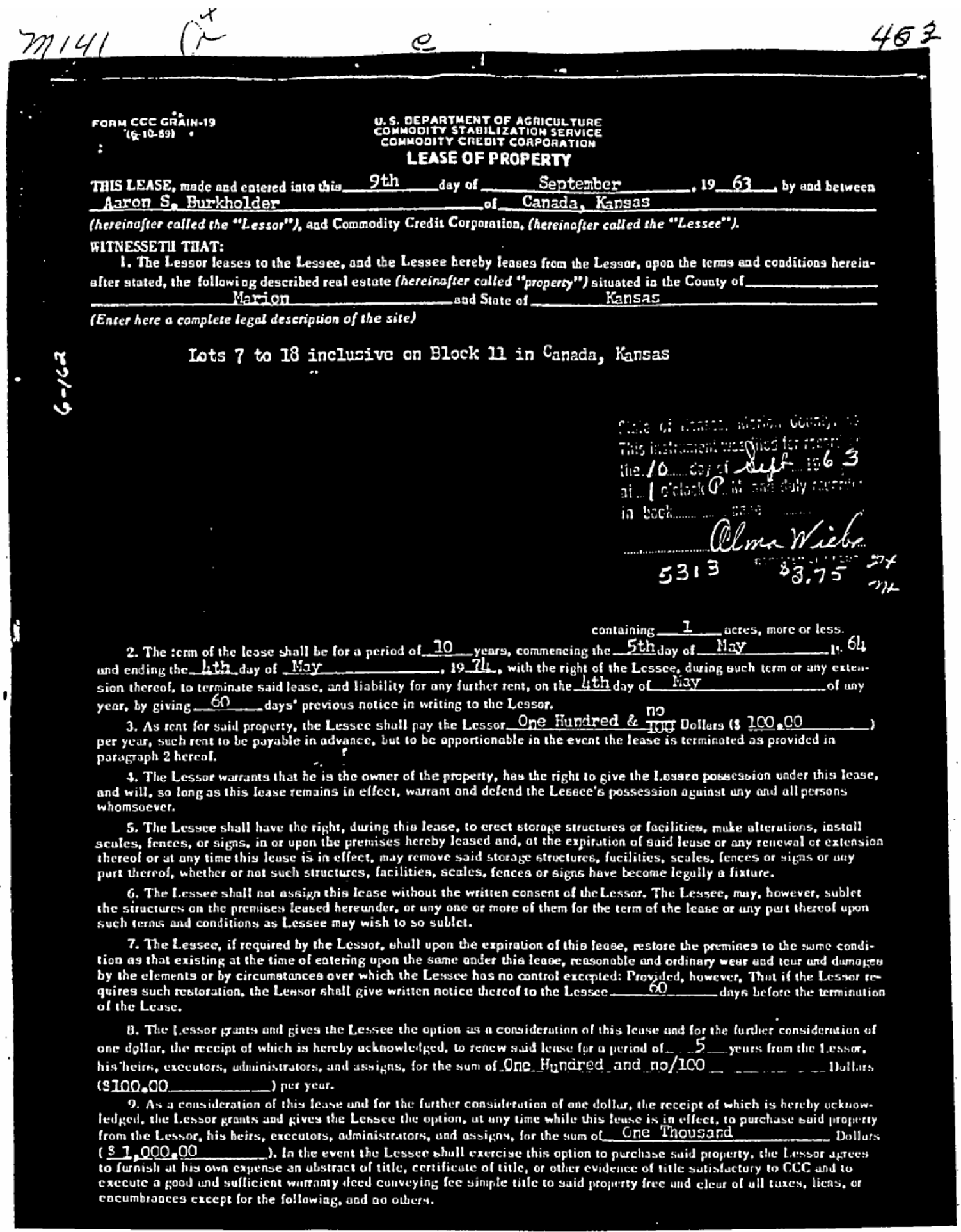

FIGURE C.2 CCC/USDA lease, 1964 to 1974. 
10. Is the event any increased tax nssessmeat is made agaiast the Lessor or the property hy virtue of the erection of starage structures and lacilities thercon by the l.essec, the l.essaz agrees to caoperate lully ia nay contest of such increased assessmeat which the l.esser fecls should he made. The lessere ngrees that the reatal hercuniler fhull he adjusind upiward lyy termined to be lecalfy increased tax assessmeat which the l.essor and Lessee mutually apree to be proper or which is de.

11. No member of of Delegate to Coapress or Resideat Commissioner, shall he admitted to ony whare or part of this Iras of purchase or to any bevefit that may arise therefrom, but this provision shatl got be construcd tn crtcal to this lease or purchase if made with a corporation for its general benefit.

12. The Lessor warrants that he has not employed any person to solicit or socure this lease upon ady afrecment for n enmmission, percentage, brokerage, or contingeat fee nad that ao such consiferntion or paymegt has bere or will he made, Bersch

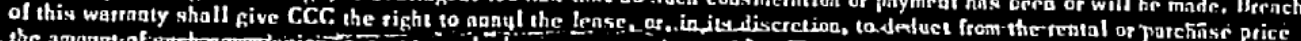

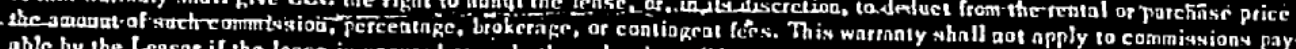
able by the tessor if the Irase is necured or made through a hema-lide ngent maintained hy the I.ensor for the purpose of
leasiag or selling his property.

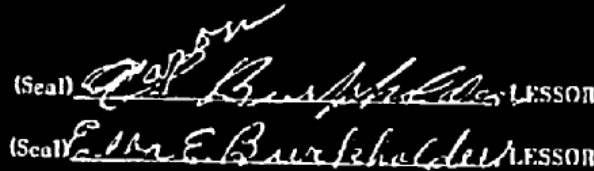
. WITHF:SS

\section{COMMODTY CREDTT CORPORATION. LESSFE:}

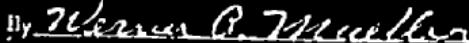

Chaiman. 2tarenter Contracting Ollices

\section{ACKHDGLEDS:MESTS}

I. Sham E, tiji 17iningm

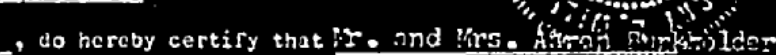
to ae known to be the persun (or persons) who exceuted the rorcgoing instrucent, personally appeared before ae and acknoivledged that he (she ar they) exceuted the same as his (her or

their) free act and deed and, in case said instrument was exceuted on behair of a corporatian, that he (she or they) as

(insert name of officer(s) and his (her or their) africial

\section{$\operatorname{titie(s))}$}

(Name of Corporation)

was (were) duly authorized by the Board

of Directors of jaid corporation to exccute the said instrucent on behalf of said corporation and to affix the corporate scal thercto.

Given under ay official hand and seal this day of Fenteminon $n$

thy commission expires

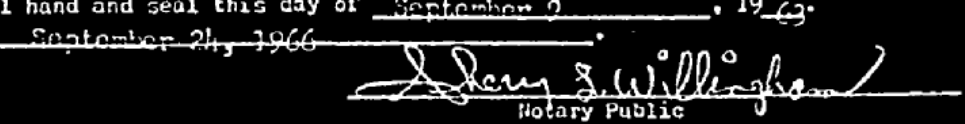

CERTIFICATIOH OF TRUF COPY

Tho undersigned hereby certifies that the foregoing tease of Property is true, correct and authentic capy of an arizinal lease duly executed by the lessar as above sut forth.

Recording official ar llatary Public

RECEIPT OF COUHTY RECORDIHG OFFICIAL

Tho above tease of Property or a true copy thereof was recorded or filcd for record on - 19 in (Chatel inortgaze or real estate records, or other) - Ho. (If Filed) 


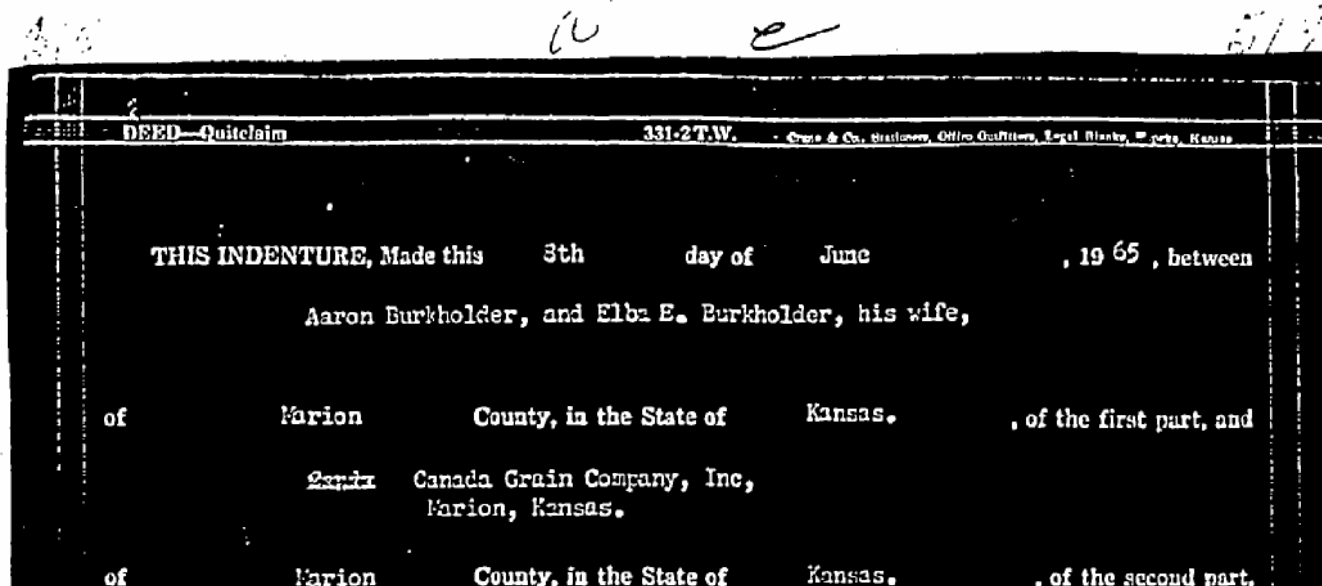

WIINESSETII, That said partics of the firat part, in consideration of the sum of

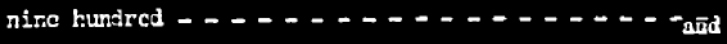

the receint of which is hereby acknowledged, do by these presents, REMISE, RELEASF AND QUITCLALS, unto said partices of the second part, its heirs and assigns, all the following-liescribed REAL ESTATE, situated in the County of ":arion

if and State of tonses. to wit:

Lot $7,3,9,10,11,12,13,14.15,10,17$, anci 13,

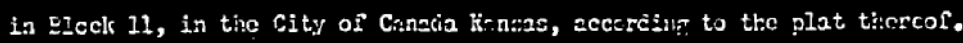

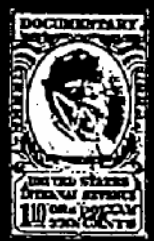

TO HAVE AND TO HOLD THE SAME, Together with all and singular the tenements, herculitaments and appurtenances thereunto belonsing, or in ang wise appertaining, forever.

IN WITNESS WHEREOF. The said part ies of the first part ha ve hereunto set thcir band $5_{\text {, }}$ the day and year firat above writtan.

Exccutud and defivercd in preseace of

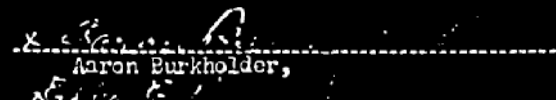

FIGURE C.3 Sale of property by Burkholder to Canada Grain Co., 1965. 


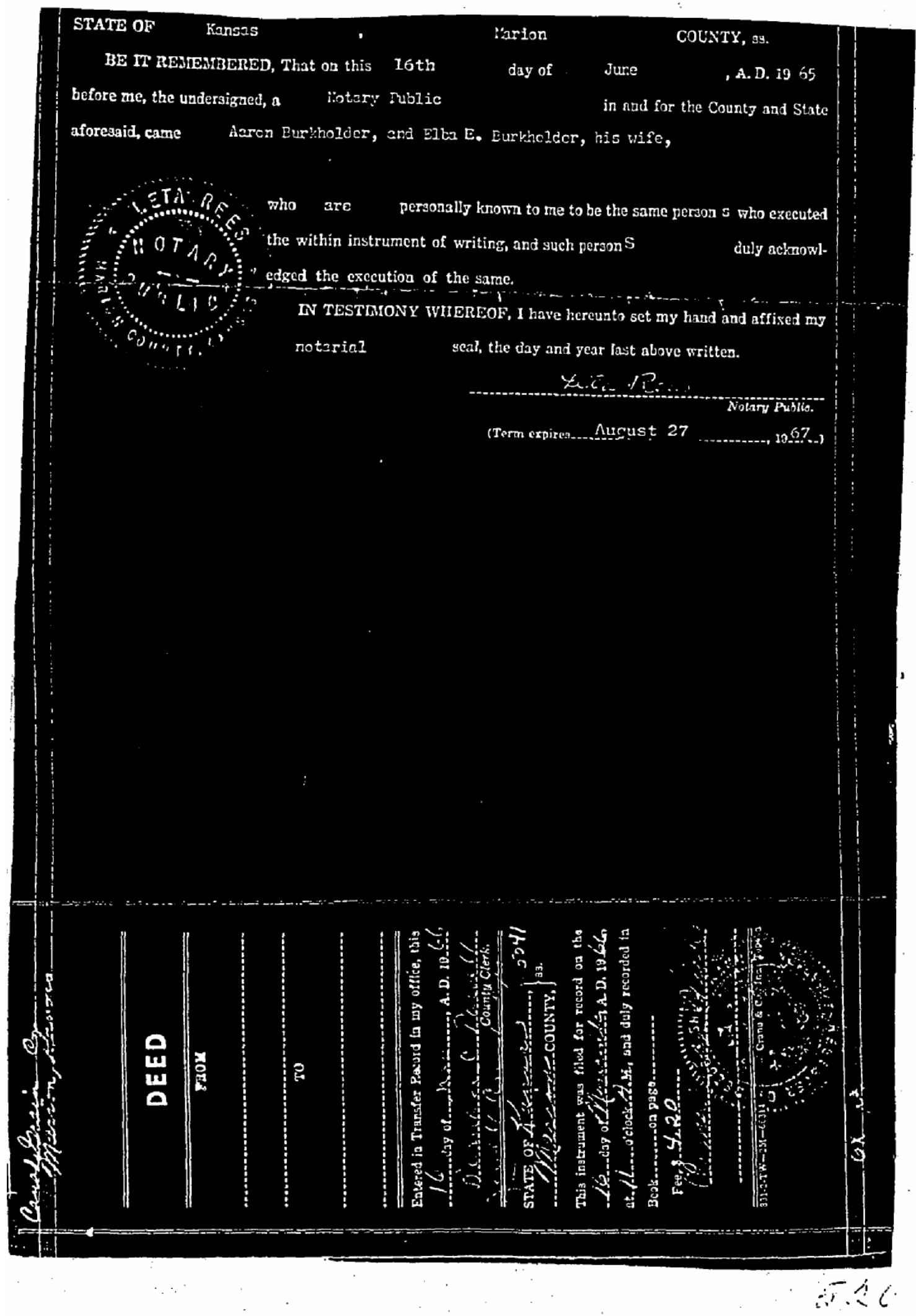

FIGURE C.3 (Cont.) 


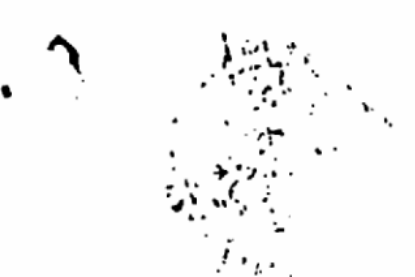

KNON ALL ME:! BY TIIESE PRESENTS, That

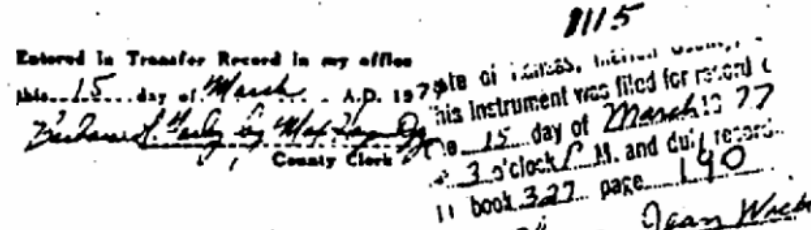

UNITED STATES MARSIIAL'S DEED

$$
11 \text { book } 3 \text { ? }
$$

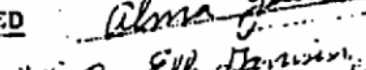

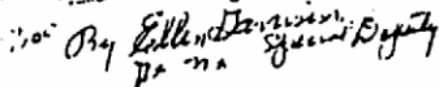

Wi!EREAS, on the 21st cloy of May, 1976, in the United States

District Court for the District of Kansas, in a certain action

then pending in saia court, numbered 76-27-C6, wherein the United

States of Arterica was plaintiff, and Canada Grain, et al.., were

defendants, a Journal Entry of Judgment and Foreclosure was rendered

in said court foreclosing the mortgage in favor of the plaintiff,

United Statcs of America, in and upon the property hereinafter des-

cribed; and it was further ordered that said property be sold by

the United States Marshal for the payment of said judgment and

costs, according to law, and that no partios were entitled to or

granted rights of redomption in said property; and

WhiEREAS, afterward, there was 1ssuad by the clerk, an Order

of Sale pirsuant to said jucigment, and in accordance with law,

eirected to the Unitec States Marshal for the District of ransas,

cormanding $\mathrm{him}$ to acivertise and sell said property according to

law, arce thot the frocepds thercef be applice as follows:

1. To the costs of this action and sald sale;

2. All taxcs unpaid and matured, and special assossments levied against said land and

real estate that are due and unpald at the date of sale and that have priority over the nortgage herein being foreclosed;

3. The cxpenses, int erest and indebtedness fcund heretofore to be owing frow the defenclants Canado Grain Co.. Inc.. Harry Helmer, Frances Heliacr, Elmor E. Rempel and Edith Rempel, to the plaintiff and also found to be a lien upon the property in question:

4. The balurec thircof, $1 f$ any, be brought into the court to awalt its further orcinr.

and thot ras raben return of the order of sale within sixty (60)

Lays fron the date tleceof, with his proceedings endorsed thereon, which Ordar of sale was delivered to and received by the waid tinlted states Marshisl on June 7, 1976, and tho said unitod states tarshal by virtue thereof, then advertisnd said property for sale

FIGURE C.4 Foreclosure on Canada Grain Co., 1976. The property includes the former CCC/USDA facility. 
by causing a notice that he would on the 13th day of July, 1975, at the frent de' $r$ of the Marion County Courthouse, Mariol:, inassas, cffer saicl lands and tenements at public sale, and sell the sare to the highest bidder at the time of sale, the said notice being published in a newspaper printed in barion Cointy, Kansas, and of general circulation in said county, the Marion County Record, which said newspaper has been continuously and uninterruptedly published in said county during the period of fifty-two (52) consecutive weaks inwediately prior to the first publication of the Notice of thla sale, sald notice being published for more than thirty (30) days impediately prior to the date of said sale; and

kHERers, on the date so named In said notlce and at the time designated therein, the Cnited States Marshal did publicly offas for sale and sell the hereinafter described real estate, tenements ard fixtures there. , to Cooperative Grain and Supply, Rillsboro,

F liansas, for the priec of $\$ 2,000.00$, to wit:

Item 17: Lots 7-12 inclusive and Lots 16-18 inclusive, all in Block 6, Canada, Kansas (Marion County);

Item 18: Lota 7-18 inclusive in Block 11 in the clty of Canada, Kansas, according to the plat thereof. (Marion County);

Item 19: Lots 1-6 inclusive of Block 3 and Lots 1-6 inclusive of Block in in original rown of Canada, Kansas. (Marion County);

Item 20: Lots 1 and 2 and the West $1 / 2$ of Lots 13,14 and 15, Block 6, Canada, Xansas. (Marion County).

and

MIEREAS, the said United States Marshal duly returned said order of sale to said court, with his proceedings thereunder endorsed thereon, ind thereafter, and on tho $21^{\text {'th }}$ day of 1973 , the court duly confirmed sold sale as having been made in all respects

FIGURE C.4 (Cont.) 


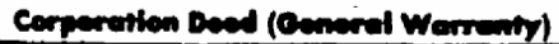

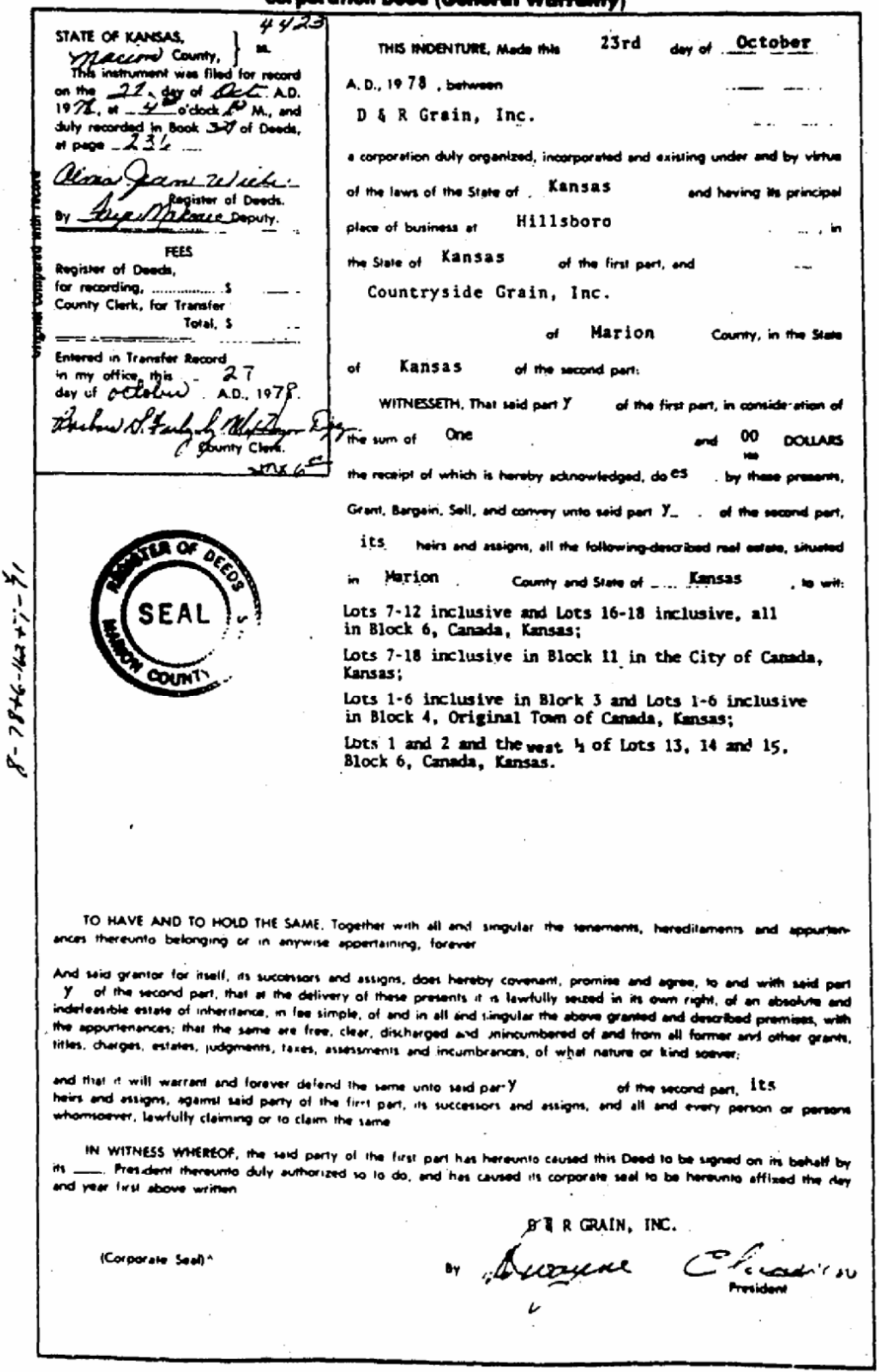

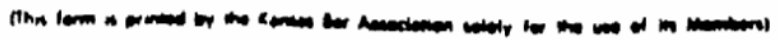

FIGURE C.5 Warranty deed, D\&R Grain to Countryside Grain, 1978. 


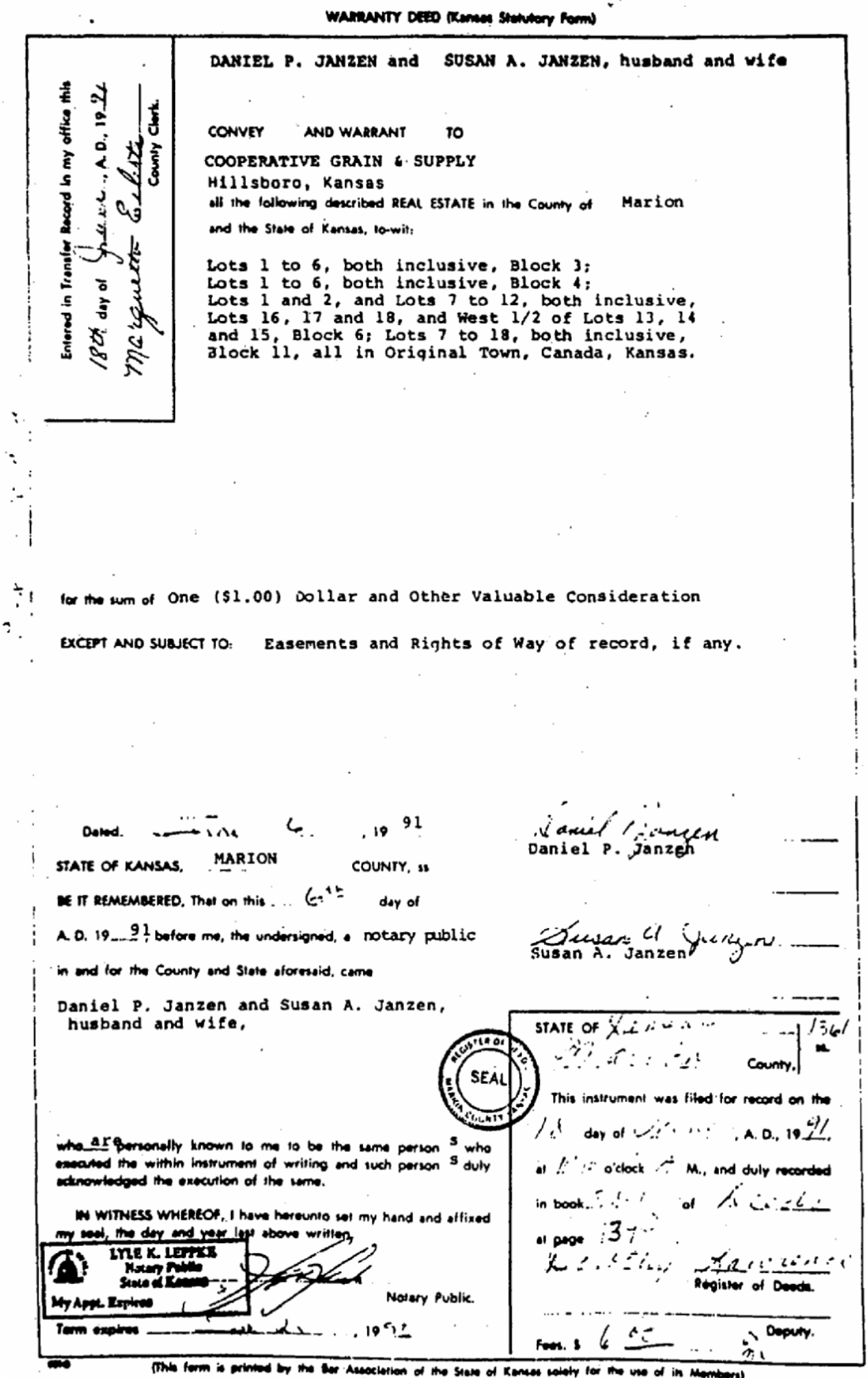

FIGURE C.6 Warranty deed, Janzen Farm Service to Cooperative Grain and Supply, 1991. 
Argonne

Environmental Science Division

Argonne National Laboratory

9700 South Cass Avenue, Bldg. 203

Argonne, IL 60439-4843

www.anl.gov

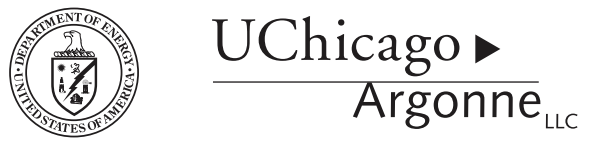

A U.S. Department of Energy laboratory

managed by UChicago Argonne, LLC 